\title{
Essais
}

ESSAIS

Revue interdisciplinaire d'Humanités

$15 \mid 2019$

Jouer l'histoire

\section{La Grande Guerre dans le jeu vidéo : jouer une histoire délaissée}

Cyril Lacheze et Marion Weckerle

\section{OpenEdition}

1 Journals

Édition électronique

URL : http://journals.openedition.org/essais/1763

DOI : $10.4000 /$ essais. 1763

ISSN : 2276-0970

Éditeur

École doctorale Montaigne Humanités

Édition imprimée

Date de publication : 15 octobre 2019

Pagination : 131-195

ISBN : 979-10-97024-07-9

ISSN : 2417-4211

Référence électronique

Cyril Lacheze et Marion Weckerle, "La Grande Guerre dans le jeu vidéo : jouer une histoire délaissée », Essais [En ligne], 15 | 2019, mis en ligne le 05 octobre 2020, consulté le 12 octobre 2020. URL : http:// journals.openedition.org/essais/1763; DOI : https://doi.org/10.4000/essais.1763 


\section{La Grande Guerre dans le jeu vidéo : jouer une histoire délaissée}

\section{Cyril Lacheze et Marion Weckerle}

Les quatre dernières années ont connu un certain regain d'intérêt de la part du public pour la thématique de la Première Guerre mondiale en général, en lien avec les célébrations du Centenaire et la médiatisation qui l'accompagne. Dans le monde du jeu vidéo français, cette communication a été accompagnée en 2014 par la sortie du jeu Soldats Inconnus : Mémoires de la Grande Guerre ${ }^{1}$, généralement mis en avant pour sa dimension éducative. À l'échelle internationale, c'est toutefois Battlefield 1 qui a retenu l'attention en $2016^{2}$, par son appartenance à la célèbre série des Battlefield, son gameplay immersif et multijoueur, et la polémique créée par l'absence des armées française et russe dans le jeu originel. Son mode multijoueur au gameplay volontairement déconnecté de la réalité de la Première Guerre mondiale lui a également valu de fréquentes comparaisons avec Verdun ${ }^{3}$, sorti l'année précédente et plus réaliste quoique moins pratiqué. Par leur coïncidence (recherchée ou non) avec l'actualité commémorative et l'écrasante sous-représentation de cette guerre par rapport à la Seconde Guerre mondiale, due entre autres à la difficulté de rendre jouable une guerre de position et à l'implication plus limitée des troupes américaines, ces trois titres sont souvent perçus comme une nouveauté qui viendrait seulement d'apparaitre dans le jeu vidéo - ce qui en fait également un argument de vente.

En réalité, il existe plus d'une centaine de titres dédiée en partie ou intégralement au conflit, les plus anciens remontant à la décennie 1970, l'ensemble formant plusieurs lignées explorant chacune certains aspects de la guerre, avec des philosophies propres. Plus précisément, en synthétisant plusieurs sources d'information et bases de données en ligne $e^{4}$, nous avons pu constituer un

1 Ubisoft Montpellier, Soldats inconnus : Mémoires de la Grande Guerre, Ubisoft, 2014.

2 Digital Illusions Creative Entertainment, Battlefield 1, Electronic Arts, 2016.

3 M2H, Blackmill Games, Verdun, M2H \& Blackmill Games, 2015.

4 On citera notamment le site Moby Games, relativement complet en termes de jeux référencés et 
corpus de 153 jeux, figurant en annexe et classés par ordre chronologique puis alphabétique (Tableau 1). Nous nous proposons ici d'en réaliser un historique critique afin de brosser un portrait plus complet et plus ancré dans le temps du traitement vidéoludique de la Grande Guerre. Cette présentation suivra trois grandes catégories typochronologiques de jeux. En premier lieu, pendant toute la période considérée, les simulateurs de vol (pour les plus anciens sous forme de side-scrollers ${ }^{5}$ ) ont constitué le socle de ces jeux, dont les plus anciens au tournant des décennies 1970 et 1980, et avec au moins un titre par année quasiment sans interruption depuis 1989, le tout représentant plus d'un tiers du corpus. Parallèlement, d'autres genres se sont développés à partir du milieu des années 1980 jusqu'au milieu des années 2000, et en particulier les jeux de stratégie (notamment les wargames ${ }^{6}$ ), doublés d'une exploration ponctuelle de nombreux autres genres à partir de 1992 ; cet ensemble regroupe environ un quart des jeux identifiés. Enfin, alors que les simulateurs de vol semblent quelque peu s'essouffler et que les wargames ont fait place aux jeux de grande stratégie ${ }^{7}$, ces nouveaux genres ont acquis une importance grandissante depuis une dizaine d'années, cette dernière période couvrant à elle seule $40 \%$ du corpus ; une part de cette dynamique est liée à l'ouverture de la plate-forme de distribution Steam ${ }^{8}$ à des jeux développés par des amateurs, d'une qualité très variable, mais qui n'en méritent pas moins d'être pris en considération. Faute de pouvoir détailler le traitement de tous les éléments historiques dans le cadre d'un article, nous nous focaliserons sur les armements présents dans chaque titre, données facilement synthétisables, également regroupées en annexe et commentées en conclusion.

Nous ne traiterons ici que des titres consacrés dès l'origine à la Première Guerre mondiale. Ceci exclut les mods, correspondant à des modifications apportées à un jeu produites indépendamment et bénévolement par des utilisateurs : il ne s'agit donc pas de jeux à part entière, mais l'ampleur des changements apportés peut dans certains cas modifier radicalement l'expérience

de détails fournis pour chacun : Blue Flame Labs., Moby Games, 1999, 2018. En ligne : https:// www.mobygames.com.

5 Un side-scroller correspond à un jeu présentant une scène en deux dimensions vue de côté, dans laquelle le personnage contrôlé par le joueur avance généralement de gauche à droite en éliminant les obstacles ou adversaires sur son chemin. Dans le cas des jeux présentés ici, il s'agit de simulations de combats aériens adoptant le même type de présentation et de gameplay, mais l'avion joué peut habituellement se déplacer à la fois vers la droite ou vers la gauche, la dénomination de side-scroller n'étant donc pas à prendre exactement au sens propre.

6 Les plus proches des jeux de stratégie sur table, les wargames tour par tour, font intervenir des unités se déplaçant sur un maillage habituellement hexagonal, chaque joueur jouant à tour de rôle.

7 La grande stratégie correspond à la gestion de larges unités, voire d'armées entières, sur des territoires importants de la taille au moins d'un pays, sans intervention du joueur dans les détails des batailles, voire des campagnes en elles-mêmes.

8 Valve Corporation, Steam, 2003, 2018. En ligne : https://store.steampowered.com/. 
de jeu. Des mods concernant la Grande Guerre, parfois très travaillés, existent pour un certain nombre de titres concernant originellement des périodes militaires relativement proches. Leur défaut général réside dans la nature même des mods, qui ne peuvent modifier en profondeur les mécaniques de jeu, et impliquent donc de conserver un fonctionnement des affrontements typiques des autres conflits. Sans surprises, la très grande majorité traite originellement de la Seconde Guerre mondiale et est donc nettement conçue autour de la notion de guerre de mouvement, qu'il s'agisse de STR ${ }^{9}$ comme Company of Heroes (mod: The Great War 1918) ${ }^{10}$ ou Men of War: Assault Squad 2 (mod: A Great War 1914-1918) ${ }^{11}$; du jeu de grande stratégie Hearts of Iron III: Their Finest Hour (mod: WW1 Mod) ${ }^{12}$; du wargame tour par tour Panzer Corps (mod: Kaiserschlacht $)^{13}$; ou encore du FPS ${ }^{14}$ Call of Duty $2(\bmod : 1914)^{15}$. Ce décalage dans les tactiques est encore plus flagrant pour ArmA II (mod: Over the Top) ${ }^{16}$ et ArmA III (mods: WW1 MOD ou Blood Trench 1914-1918) ${ }^{17}$, qui traitent à l'origine du combat contemporain, mais avec une immersion notoire expliquant leur présence ici. Pour des périodes plus anciennes que la Première Guerre mondiale, on peut citer Napoleon : Total War (mod: The Great War $)^{18}$, où, dans le mode tactique ${ }^{19}$, les troupes avancent en masses compactes et s'adaptent difficilement aux " tranchées $»^{20}$; et beaucoup plus éloigné encore, Mount and Blade: Warband (mod: Iron Europe) $)^{21}$, avec une thématique médiévale-fantastique. Dans tous les cas, si les résultats sont rarement parfaitement adaptés à une représentation de la Première Guerre mondiale et

9 Jeu de Stratégie en Temps Réel. Type de jeu où le joueur contrôle une faction (par exemple une civilisation entière) devant en affronter militairement d'autres, et dont le gameplay se déroule de façon continue (par opposition au jeu de stratégie en tour par tour). On citera dans les fondateurs du genre Dune II (1992), Warcraft (1994), Command and Conquer (1995), Age of Empires (1997) ou encore StarCraft (1998).

10 Relic Entertainment, Company of Heroes, Sega, 2006.

11 Digitalmindsoft, Men of War: Assault Squad 2, 1C Company, 2014.

12 Paradox Development Studio, Hearts of Iron III: Their Finest Hour, Paradox Interactive, 2012.

13 The Lordz Games Studio, Flashback Games, Panzer Corps, Slitherine Strategies, 2011.

14 First-Person Shooter, en français Jeu de tir à la première personne. Jeu de combat dans lequel le joueur contrôle un personnage en voyant le monde à travers ses yeux. L'archétype du FPS est Doom (1993).

15 Infinity Ward, Call of Duty 2, Activision, 2005.

16 Bohemia Interactive, Arma II, 505 Games, 2009.

17 Bohemia Interactive, Arma III, Bohemia Interactive, 2013.

18 The Creative Assembly, Napoleon: Total War, Sega, 2010.

19 La série des Total War fait intervenir un mode de jeu stratégique, où une carte mondiale ou régionale permet de gérer les villes et le déplacement des armées, et un mode tactique employé pour la simulation détaillée des batailles.

20 Ou plus exactement à de petites fortifications presque de surface, plutôt typiques de la guerre de Sécession. L'ensemble pourrait éventuellement convenir pour décrire la première phase du conflit mondial, mais difficilement les quatre années de guerre.

21 TaleWorlds, Mount and Blade: Warband, Paradox Interactive, 2010. 
particulièrement des combats de tranchées, l'existence de ces mods démontre nettement l'intérêt des joueurs pour ce conflit, certains étant prêts à s'investir considérablement pour adapter leurs jeux favoris à cette période.

\section{Simulateurs de vol}

Les plus anciens jeux identifiés se donnant pour cadre la Première Guerre mondiale sont des side scrollers sur borne d'arcade ${ }^{22}$ dans lesquels le joueur incarnait un pilote d'avion, respectivement BiPlane ${ }^{23}$, BiPlane $4^{24}$ et Wings ${ }^{25}$, sortis en 1975 pour le premier et 1976 pour les deux suivants. BiPlane et Wings se jouaient à deux, tandis que BiPlane 4 permettait de jouer à quatre. Dans ces premiers jeux, les joueurs pouvaient identifier leur avion sur l'écran par sa couleur, mais celle-ci ne renvoyait pas à une nationalité particulière ; pour gagner, le joueur devait remporter autant de duels que possible avant la fin du temps de jeu alloué. Le gameplay était donc extrêmement simple, adapté au support de la borne d'arcade, et son principe de duel aérien était concrètement, avec la représentation d'avions biplans, l'élément utilisé le plus emblématique de la Première Guerre mondiale. Le premier simulateur de vol proprement dit, dans lequel le joueur appréhendait le jeu par une vue à la première personne, gérer sa mission et les paramètres de pilotage, fut la borne d'arcade Red Baron de $1980^{26}$. Le jeu était visuellement très simple, et le décor - en l'occurrence les deux mitrailleuses du joueur - se trouvait directement sur la borne et non pas sur l'écran. Le jeu simulait des duels aériens, ou dogfight, contre d'autres avions et des dirigeables au moyen d'une animation vectorielle; le joueur avait également la possibilité de bombarder des cibles au sol. Les autres simulateurs de vol développés sur seulement trois années consécutives dans la décennie 1980 - Air Battle ${ }^{27}$, Blue Max ${ }^{28}$, Flying Ace ${ }^{29}$, et Snoopy and the Red Baron en $1983^{30}$, deux versions de Sopwith en 1984 et $1985^{31}$, et enfin Red Baron ${ }^{32}$ et Sky Kid en $1985^{33}$ - étaient tous des side scrollers sur

22 Les jeux d'arcade sont globalement des jeux de tir faisant uniquement appel aux réflexes et à la précision du joueur pour détruire des adversaires, sans aucune notion de simulation ou de stratégie. Ce terme peut être employé par extension comme adjectif pour qualifier un jeu ou un mode de jeu.

23 Fun Games, BiPlane, Fun Games, 1975.

24 Fun Games, BiPlane 4, Fun Games, 1976.

25 Electra Game, Wings, Electra Game, 1976.

26 Atari, Red Baron, Atari, 1980.

27 Anonyme, Air Battle, Philips Export B.V., 1983.

28 Synapse Software, Blue Max, Synapse Software, U.S. Gold, 1983.

29 Microcomputer Games, Flying Aces, The Avalon Hill Game Company, 1983.

30 Atari, Snoopy and the Red Baron, Atari, 1983.

31 BMB Compuscience, Sopwith, BMB Compuscience, 1984. BMB Compuscience, Sopwith, BMB Compuscience, 1985.

32 B. D. Hambüchen, Red Baron, Labochrome, 1985.

33 Namco, Sky Kid, Namco, Sunsoft, 1985. 
consoles avec un gameplay similaire, consistant à détruire des avions ennemis et des cibles au sol. La Première Guerre mondiale servait d'habillage et d'inspiration pour des skins d'appareils simples ainsi que pour des noms de jeu accrocheurs, mais ne constituait pas un élément structurant des jeux - Sopwith comportait d'ailleurs des skins de chars de la Seconde Guerre mondiale. Dans Blue Max, le joueur pilotait un avion remplissant à la fois le rôle de chasseur et celui de bombardier, ce qui ne correspond pas aux missions historiques. Flying Ace intégrait cependant un aspect du conflit rarement abordé dans les simulateurs de vol du corpus, toutes périodes confondues : le but était de détruire la chaîne d'approvisionnement de l'ennemi, alors que la majorité des jeux se concentrent sur le duel aérien.

Il faut noter particulièrement dans cet ensemble deux jeux à l'approche décalée. Snoopy and the Red Baron (1983) s'inspirait des bandes dessinées de Charles Schultz, dans lesquelles le chien Snoopy imagine être un aviateur de la Première Guerre mondiale, avec pour ennemi récurrent dans les airs le Baron Rouge (Manfred von Richthofen, as de l'aviation allemande durant la guerre). Il s'agissait là du premier simulateur de vol dans lequel le contexte de la Première Guerre mondiale était essentiel dans le concept du jeu, même si l'action se déroulait dans l'imagination de Snoopy. C'était également le premier jeu identifiant clairement le camp du joueur - celui-ci contrôlait Snoopy qui, comme dans la bande dessinée, se bat côté allié, et le but du jeu était de vaincre le Baron rouge. Toutefois, si les autres jeux se concentraient finalement sur la représentation et la simulation d'avions rappelant le conflit, dans Snoopy and the Red Baron le joueur contrôlait la niche de Snoopy comme simulacre immobile de chasseur Sopwith Camel, comme dans la bande dessinée. Il s'agissait également du premier jeu identifiant clairement le célèbre as allemand comme un ennemi à abattre, même si de nombreux titres incluaient son surnom. En effet, dans les Red Baron de 1980 et de 1985, le joueur n'incarnait pas de personnage en particulier : le nom servait seulement à suggérer qu'il s'agissait de jeux de duel aérien. Snoopy and the Red Baron plongeait donc le joueur dans la Première Guerre mondiale de manière fantaisiste - il s'agissait de la version rêvée par Snoopy - mais parfaitement cohérente avec l'imagination du personnage et la bande dessinée d'origine. Par ailleurs, Sky Kid (1985) s'en rapprochait légèrement par son univers coloré et le fait d'incarner des animaux dans le jeu.

Le début des années 1990 vit le développement de simulateurs de vols nettement plus poussés, à partir de War Eagles en 198934, dans lesquels la manœuvre de l'appareil en elle-même prit une place plus importante, pour exploiter les possibilités de jeux plus réalistes ${ }^{35}$; la part des side scrollers s'ef-

34 Cosmi Corporation, War Eagles, Cosmi Corporation, 1989.

35 Artech Digital Entertainment, Blue Max: Aces of the Great War, Three-Sixty Pacific, 1990. 
fondra en conséquence et presque immédiatement, le genre disparaissant dès $1992^{36}$. La plupart furent structurés sous la forme d'un certain nombre de missions pour le joueur, avec des récompenses sous la forme de montée en grade dans le jeu, et de duels aériens. La variété des aéronefs jouables augmenta également significativement : par exemple, Knights of the Sky comporte vingt avions $^{37}$, et le Red Baron de 1990, vingt-huit ${ }^{38}$. Ce dernier jeu se présentait par ailleurs comme historiquement correct : non seulement divertissant pour le joueur, mais avec l'ambition de restituer la réalité du vol durant la Première Guerre mondiale, avec un aspect survival. Il comportait en effet une chronologie et le but était, pour le joueur, de survivre jusqu'à la fin de la guerre, élément de gameplay qui n'était pas possible dans les jeux d'arcade. Le joueur accédant à des appareils plus performants au fur et à mesure de sa progression dans le jeu, ce dernier intégrait également la notion de progrès technologique de l'aviation. Knights of the Sky utilisa une variante de la même idée, avec un mode de jeu Première Guerre mondiale dans lequel le joueur, aviateur débutant en 1916, devait survivre et accéder au statut d'as. Ce titre permettait de choisir, dans le "hangar ", un appareil parmi les avions développés durant la timeline du jeu, imitant également la temporalité réelle de la disponibilité des modèles. De plus, la notion de balistique y était présente : le gameplay nécessitait de prendre en compte la trajectoire descendante des balles et de se positionner un peu au-dessus de l'ennemi pour tirer. Dawn patrol: Head to Head $(1995)^{39}$ inscrivit également les joueurs dans l'imaginaire nourri de l'histoire des as, puisque le jeu comportait une campagne dite américaine dans laquelle on incarnait un as états-unien ayant combattu en France, Eddie Rickenbacker. Une autre permettait d'incarner le pilote allemand Lothar von Richthofen (frère de Manfred). À l'instar des jeux Snoopy, un duel contre le Baron Rouge avait lieu dans Warbirds $(1991)^{40}$ et Wings 2 Aces High $(1992)^{41}$, alors même que le reste du jeu était concentré sur du bombardement. En 1990, Chocks away ${ }^{42}$ récompensait le joueur par la possibilité de monter en grade, le plus haut atteignable dans le jeu étant le grade Marshall of the Royal Air Force (corps d'aviation britannique). Si l'histoire des as était donc utilisée pour son volet héroïque ainsi que pour l'ancrage du jeu dans un contexte

Vektor Grafix, Air Duel: 80 Years of Dogfighting, MicroProse, 1993. Rowan Software, Dawn Patrol, Empire Interactive, 1994. Rowan Software, Flying Corps, Empire Interactive, 1996. Aqua Pacific, ACM 1918, Project Two Interactive BV, 1997.

36 Richard Ling, Dogfight, Amiga, 1990. Steven Metcalf, Blue Baron, Zeppelin Games Limited, 1992.

37 MicroProse, Knights of the Sky, MicroProse, 1991.

38 Dynamix, Red Baron, Sierra On-Line, 1990.

39 Rowan Softwar, Dawn Patrol: Head to Head, Empire Interactive Entertainment, 1995.

40 Atari, Warbirds, Atari, 1991.

41 Malibu Interactive, Wings 2: Aces High, Namco, 1992.

42 Andrew Hutchings, Chocks Away, The Fourth Dimension, 1990. 
historique, a contrario, The Ancient Art of War in the Skies (1993) constitua une exception ${ }^{43}$ : à l'instar de The Ancient Art of War développé l'année suivante par la même équipe et fondateur dans le genre des STR, il s'agissait d'un jeu comprenant plusieurs campagnes inspirées des événements de la guerre, mais ne cherchant pas à restituer quelque chose d'historique ni même de cohérent, au profit de l'aspect stratégique exclusivement.

Red Baron comportait de plus une différenciation graphique crédible des différents types d'appareils selon leurs missions, avec les marquages nationaux et les couleurs réelles connues. Les avions du jeu étaient connus du grand public et revinrent régulièrement dans les jeux ultérieurs jusqu'aux plus récents : les Sopwith Camel, le Fokker Dr.III rouge de Manfred von Richthofen, les Spad XIII français, les Gotha allemands pour les missions de bombardement. Certains effets attestés dans la manœuvrabilité des appareils furent également inclus pour la première fois, comme un enrayement de mitrailleuse pouvant effectivement survenir dans le jeu, un atterrissage raté où le nez de l'avion se plante dans le sol en cheval de bois, ou l'impossibilité de voir un ennemi arrivant de face avec le soleil dans le dos avant qu'il ne soit extrêmement près, manœuvre bien connue de la célèbre escadrille allemande Jasta 11. Le jeu prévoyait par ailleurs la possibilité de jouer des missions " historiques ». Le succès fut suffisant pour que trois autres jeux Red Baron soient réalisés à la fin de la décennie 1990 ${ }^{44}$. Wings en 1990 (remastérisé en 2014) ${ }^{45}$ employa également beaucoup d'éléments historiques, avec la volonté d'ancrer le jeu dans une histoire hérö̈que pour solliciter les émotions du joueur et augmenter son investissement dans le gameplay. Le manuel du jeu était émaillé de citations d'écrits d'as célèbres comme Edward « Mick » Mannock, fournissait des fiches biographiques, des fiches d'identification des aéronefs, ainsi que des explications sur les principes de base du vol ${ }^{46}$. Contrairement aux simulateurs ultérieurs, celui-ci donnait une vue à la première personne depuis le cockpit, mais avec le haut du corps du pilote incarné représenté aux commandes; sa tête se tournait du côté duquel allait venir le prochain aéronef ennemi, donnant ainsi une assistance visuelle au joueur. Celui-ci, dans les simulateurs, contrôlait habituellement l'appareil par une vue à la première personne depuis l'intérieur du cockpit de l'avion, avec un tableau de bord plus ou moins détaillé sur l'écran ; les jeux ne permettaient cependant pas de manœuvrer l'avion en fonction des indications données par les jauges, celles-ci reproduisant au contraire les choix du joueur.

43 Evryware, The Ancient Art of War in the Skies, MicroProse, 1993.

44 Dynamix, Red Baron II, Sierra On-Line, 1997 ; Dynamix, Red Baron 3-D, Sierra On-Line, 1998 ; Dynamix, Curse You! Red Baron, Sierra On-Line, 1999.

45 Cinemaware, Wings, Cinemaware, 1990.

46 Anonyme, Wings, Cinemaware, 1990, 76 p. 
Warbirds, en $1991^{47}$, fit la synthèse des caractéristiques principales des jeux de vol de l'époque se déroulant pendant la Première Guerre mondiale, puisque le joueur pouvait choisir entre deux modes : arcade et simulation. Quant à Wings of Glory $(1994)^{48}$, il permettait au joueur de choisir le niveau de réalisme qu'il souhaitait appliquer : la possibilité que la mitrailleuse s'enraye, la possibilité de dégâts sur les ailes, ou encore celle d'être ébloui par le soleil. Le joueur ne disposait en revanche d'aucune indication sur la direction de laquelle pouvaient venir les avions ennemis et devait donc surveiller attentivement les côtés de son cockpit, ainsi que devant et derrière lui. Il réunissait en conséquence les possibilités de simulation les plus réalistes en regard des possibilités des ordinateurs, jusqu'à Red Baron 3-D $(1998)^{49}$, que l'on peut considérer comme un archétype de simulateur de vol. Bien que quelques appareils français y apparaissent, ces simulateurs de vols se centraient sur les aéronefs britanniques et allemands, avec les Allemands souvent antagonistes par défaut. Par exemple, Wing nuts: Battle in the Sky en 1997 plaçait résolument le joueur dans le rôle d'un volontaire américain en France, ne laissant pas le choix au joueur ${ }^{50}$.

L'édition de jeux de vols se déroulant pendant la Première Guerre mondiale fut quasiment ininterrompue depuis le début des années 1980, et c'est un constat que l'on peut faire également pour les années 2000 et le début des années 2010. Les deux genres majoritaires restèrent les mêmes : l'arcade et le simulateur " réaliste ", avec une nouveauté importante, à savoir le développement du jeu en ligne (et de la mise en ligne de productions amatrices, plutôt expérimentales ${ }^{51}$ ). On rencontrait ainsi dès 2000 Aces High $^{52}$, dont la troisième itération date de $2016^{53}$, jeu en ligne massivement multijoueur comprenant parmi le matériel jouable (navires, aéronefs et matériel terrestre) quatre avions de la Première Guerre mondiale, et des simulateurs de plus en plus poussés à partir de $2006^{54}$ avec notamment First Eagles: The Great War 191855. La simulation réaliste de manœuvres de plus en plus d'avions, tant au niveau des graphismes que du comportement des avions et des paramètres à prendre en compte, en constituaient l'essentiel du gameplay : sur les titres les plus complets, il devenait ainsi nécessaire de prendre en compte la résistance structurelle de l'avion, les forces d'accélération pouvant faire perdre connaissance

47 Atari, Warbirds, Atari, 1991.

48 Origin Systems, Wings of Glory, Electronic Arts, 1994.

49 Dynamix, Red Baron 3-D, Sierra On-Line, 1998.

50 Rocket Science Games, Wing Nuts: Battle in the Sky, BMG Interactive Entertainment, 1997.

51 Ilan Papini, John Marco, Canvas Knights, Ilan Papini, John Marco, 2015.

52 HiTech Creations, Aces High, HiTech Creations, 2000.

53 HiTech Creations, Aces High III, HiTech Creations, 2016.

54 First Class Simulations, WW1 Fighters, First Class Simulations, 2007. First Class Simulations, Mission: WWI Dogfight, Abacus Software, 2008.

55 Third Wire Productions, First Eagles: The Great War 1918, G2 Games, 2006. 
au pilote, le vent, et la totalité des éléments mécaniques intervenant réellement dans ces appareils. Le nombre d'avions jouables s'enrichit également considérablement ; Rise of Flight: The First Great Air War ${ }^{56}$ et Wings over Flanders Fields $^{57}$, toujours largement joués actuellement, sont les simulateurs disponibles les plus riches en nombres d'avions, avec plus de trente appareils jouables (dont plusieurs variantes de certains modèles, comme les quatre versions du Nieuport 24 dans Wings over Flanders Fields), en plus de faire partie des plus complets et d'être multijoueur (pour le premier). En outre, il y est possible de jouer des avions dédiés à la reconnaissance, au bombardement, ou multi rôles, en plus des avions de chasse classiques. Ainsi, on y trouve des modèles d'avions célèbres et omniprésents dans les jeux du corpus, et ce indépendamment du support ou de l'année de sortie du jeu, tels le Spad XIII, ou le triplan Fokker Dr. I présent dans vingt-deux jeux, mais aussi des avions beaucoup plus rares comme des Aviatik allemands ou encore le Bréguet 14, dévolu à la reconnaissance côté français. Rise of Flight: The First Great Air War s'enrichit pour sa part en appareils au fur et à mesure des mises à jour, ce qui allonge la durée de vie du jeu et lui permet de proposer des avions inexistants dans les autres simulateurs, comme quelques hydravions. Si les appareils français sont un peu plus représentés, l'attention reste tout de même très majoritairement portée sur les aviations britannique et allemande, l'Autriche-Hongrie, l'Italie, la Russie et les pays perçus comme " mineurs " étant ainsi totalement absents ou presque, alors qu'ils ont tous déployé des pilotes dont certains ont atteint le rang d'as ${ }^{58}$. On soulignera tout de même le jeu Ilya Muromets $(2015)^{59}$ centré sur le bombardier russe éponyme, par ailleurs étrangement sous-représenté par rapport à sa notoriété. Ainsi, depuis le début des années 2000, les simulateurs "réalistes » s'orientent globalement vers la complexification du jeu dans une recherche de réalisme, et vers la multiplication des matériels jouables.

L'autre genre majoritaire de jeu d'avions de la Première Guerre mondiale est l'arcade, avec un niveau de Crash Bandicoot 3: Warped (1998) ${ }^{60}$, Wings of War (2004) $)^{61}$, Sky Aces $(2006)^{62}$, Ace of Aces $(2008)^{63}$ ou encore Air Conflicts: Secret Wars $(2011)^{64}$. À l'instar des jeux d'arcade des décennies précédentes, le réalisme y était plus qu'accessoire : dans Wings of War, le joueur pouvant lancer des roquettes et se protéger avec un bouclier, ce qui n’a évidemment

56 Neoqb, 777 Studios, Rise of Flight: The First Great Air War, 777 Studios, Aerosoft, ND, 2009.

57 OBD Software, Wings Over Flanders Fields, OBD Software, 2014.

58 Ce titre est acquis à la cinquième victoire aérienne, soit au cinquième appareil ennemi abattu.

59 1C Games Studios, Ilya Muromets, 1C Games Studios, 2015.

60 Naughty Dog, Crash Bandicoot 3: Warped, Sony Computer Entertainment, 1998.

61 Silver Wish Games, Wings of War, Gathering of Developers, 2004.

62 The X Studio Productions, Sky Aces, IncaGold, 2006.

63 Anonyme, Ace of aces, s.n., 2008.

64 Games Farm, Air Conflicts: Secret Wars, bitComposer, 2011. 
rien d'historique. Nombre de ces jeux d'arcade continuaient d'utiliser l'imaginaire de la bande dessinée Snoopy ${ }^{65}$ et le symbolisme du Baron Rouge ${ }^{66}$; en 2006, Flyboys Squadron ${ }^{67}$ exploitait simplement l'intérêt provoqué par la sortie concomitante du film Flyboys de Tony Bill. Ces jeux étaient généralement structurés en un certain nombre de campagnes dans lesquelles le joueur incarnait un pilote côté allié (option la plus souvent adoptée), ou allemand. Comme dans le cas des simulateurs réalistes, l'Autriche-Hongrie, la Russie ou l'Italie n'apparaissaient jamais. On soulignera tout de même que Master of the Skies: The Red Ace était le premier jeu comportant des missions en Afrique, alors que les autres jeux aériens sur la Première Guerre mondiale avaient une localisation géographique souvent centrée sur la France et la Belgique. Le scénario d'Air Conflicts: Secret Wars, dans lequel on jouait un personnage féminin, se déroulait quant à lui durant les deux Guerres mondiales et permettait de piloter dans le jeu des appareils des deux conflits, bien que le second soit plus présent. Des genres dérivés ont également été expérimentés : en 2003 puis 2006, Wings of Honour se présentait partiellement comme un jeu d'aventure, l'histoire suivant les exploits d'un pilote britannique inventé à travers la guerre $^{68}$. Enfin, le développement des jeux gratuits sur navigateur conduisit à la réapparition du side scroller avec Dogfight: The Great War en $2008^{69}$, aux graphismes bien plus fluides et colorés que ceux de ses homologues des années 1980, mais au gameplay sensiblement équivalent.

Il faut par ailleurs signaler le récent Ace Patrol de Sid Meier $(2013)^{70}$, lequel n'entre dans aucune de ces catégories du fait de sa conception bien particulière à de nombreux points de vue, suivant en cela la tradition innovatrice de son concepteur ${ }^{71}$. En effet, il s'agit d'un jeu purement tactique, conçu pour être joué sur téléphone portable. Le joueur y manœuvre un groupe de quatre avions (qui ne s'identifient pas par un modèle particulier) au tour par tour, en prenant en compte la position des autres avions. Celle-ci influe en effet sur les mouvements possibles, tout comme des paramètres tels que le vent, l'altitude, ou les nuages, le tout obligeant en conséquence le joueur à antici-

65 Smart Bomb Interactive, Snoopy vs. the Red Baron, Namco Bandai Games, 2006. Smart Bomb Interactive, Snoopy Flying Ace, Microsoft Game Studios, 2010.

66 Fiendish Games, Master of the Skies: The Red Ace, Global Star Software, Small Rockets, 2000. Small Rockets, Red Ace Squadron, Global Star Software, 2001. Atomic Planet Entertainment Limited, Red Baron, Davilex Games B.V., 2005. Magnussoft, Flying Baron 1916, magnussoft, 2016.

67 iEntertainment Network, Flyboys Squadron, iEntertainment Network, 2006.

68 CITY interactive, Wings of Honour, CITY interactive, 2003. CITY interactive, Wings of Honour: Battles of the Red Baron, CITY interactive, 2006.

69 Rock Solid Games, Dogfight: The Great War, Rock Solid Arcade, 2008.

70 Firaxis Games, Sid Meier's Ace Patrol, 2K Games, 2013.

71 Sid Meier, icône dans l'univers de la conception de jeux vidéo, est principalement célèbre pour sa série Civilization, comptant à l'heure actuelle six titres parus entre 1994 et 2016. 
per ses actions. Ce jeu s'inscrit ainsi pleinement dans la lignée des wargames physiques dont quelques titres furent effectivement consacrés à l'aviation de la Première Guerre mondiale (comme Dogfight édité en 1962 par American Heritage), y compris sous la forme expérimentale de livrets visuels (Ace of Aces en 1980 chez Nova Game Designs), et pourtant très peu portés sur support informatique depuis Eagles en 1983 (voir ci-après).

\section{Wargames et nouveaux genres : 1983-2005}

Parallèlement à l'omniprésence des simulations aériennes, la décennie 1980 a également vu le développement relativement conséquent des jeux de stratégie sur la Grande Guerre, à commencer par des wargames, mais aussi, plus remarquablement pour une époque aussi ancienne, quelques STR ${ }^{72}$. Les deux premiers wargames furent présentés en 1983. Eagles s'inscrivait pleinement dans la mouvance de l'époque concernant les jeux sur ce conflit puisqu'il portait également sur l'aviation, thématique pourtant généralement peu présente dans les jeux de stratégie ${ }^{73}$; il était édité par la jeune société Strategic Simulations, fondée deux ans auparavant et se positionnant déjà en leader des jeux de stratégie informatiques ${ }^{74}$. Cette société adaptant directement les codes et habitudes complexes du wargame sur table à l'outil informatique, Eagles prenait déjà en compte, malgré sa date précoce, des données telles que l'altitude de vol, le vent ou les conditions météorologiques. Cette logique de portage direct de la table à l'informatique est évidente avec l'exemple de Grand Fleet en 1988 : ce jeu n'avait pas de graphismes, mais un plateau et des pions inclus dans la boîte, le joueur jouant simplement les coups indiqués par la machine sous forme de texte ${ }^{75}$. L'autre wargame de 1983, Battle 1917, s'inspirait lui plutôt des échecs, des unités terrestres grossièrement inspirées de la Première Guerre mondiale se déplaçant sur une carte générée aléatoirement, mais peu étendue, et devant capturer un roi ${ }^{76}$. La lignée des wargames très détaillés s'est poursuivie dans la seconde moitié des années 1980, malgré l'exis-

72 On peut considérer le jeu Legionnaire, dont une première version date de 1979, comme le précurseur des STR. Toutefois, le premier à avoir rencontré un succès commercial fut The Ancient Art of War en 1984 (précédemment cité) et, comme signalé dans une note précédente, les références du genre ne datent que des années 1990.

73 Paul Murray, Eagles, Strategic Simulations, 1983.

74 Strategic Simulations, Inc. (généralement et par la suite abrégé en SSI), était le concurrent direct d'Avalon Hill, éditeur incontournable de wargames sur table qui s'était également lancé sur le marché informatique (par exemple avec Flying Ace [1983], cité précédemment). Prenant le dessus sur ce dernier marché, SSI devint rapidement la référence pour les wargames informatiques extrêmement pointus et très documentés, ainsi que pour les jeux de rôle. Cette compagnie a été absorbée par Ubisoft en 2001, laquelle se tourna cependant vers des jeux destinés à des publics plus larges et abandonna donc le savoir-faire de SSI en matière de jeux de stratégie.

75 Simulations Canada, Grand Fleet, Simulations Canada, 1988.

76 Mark Lucas, Battle 1917, Cases Computer Simulations, 1983. 
tence de quelques titres moins précis ${ }^{77}$. On citera ainsi The Great War 1914, qui employait les symboles d'unités de l'OTAN (sans même les expliciter, ce code étant familier des joueurs de wargames sur table ${ }^{78}$; Halls of Montezuma ${ }^{79}$, avec un scénario ${ }^{80}$ sur la bataille du bois de Belleau ${ }^{81}$, mais aussi Battle Cruiser, un premier wargame naval édité par $S \mathrm{II}^{82}$, avec des règles si précises, détaillées et réalistes que le manuel peut quasiment être apprécié indépendamment du jeu. Avec environ 80 classes de bâtiments jouables pour les seules marines allemande et britannique, constituant d'ailleurs la première apparition d'unités clairement identifiées dans les jeux de stratégie décrits, ce titre couvrait la quasitotalité des classes de cuirassés et croiseurs ayant été en service depuis les prédreadnoughts ${ }^{83}$ et les croiseurs cuirassés de l'extrême fin du XIX ${ }^{\mathrm{e}}$ siècle, jusqu’à la fin de la Grande Guerre, ainsi qu'une sélection importante de destroyers. Ces caractéristiques ont été encore accentuées quelques années plus tard dans Dreadnoughts $^{84}$ : en plus de proposer un commandement depuis la passerelle du navire amiral, celui-ci s'appuyait sur des recherches en archives pour fournir une liste au moins aussi complète pour les marines allemande et britannique (en particulier pour les destroyers), fournissant même les détails techniques des canons et munitions, mais y ajoutant en plus les marines russe, japonaise et chinoise $^{85}$. Jutland, en 1993, semble avoir suivi la même logique ${ }^{86}$. Enfin, SSI

77 A. et O. Bishop, Gallipoli, Cases Computer Simulations, 1986.

78 Marc Summerlott, The Great War 1914, D.K.G., 1986. Anonyme, The Great War-1914. WWI Computer Strategy Game August-October 1914, D.K.G., 1986, s.p.

79 Strategic Studies Group, Halls of Montezuma: A Battle History of the United States Marine Corps, Strategic Studies Group, 1987.

80 Dans les jeux de stratégie, un «scénario " désigne une partie de jeu dont la carte, au moins certaines données sur les unités, et éventuellement des événements préprogrammés sont prévus à l'avance, permettant ainsi d'ancrer le jeu dans un contexte (historique ou non). Ce type de partie s'oppose à un jeu libre dans lequel les conditions de départ sont plus aléatoires et le déroulement du jeu laissé à la pleine appréciation du joueur.

81 Importante bataille menée par les troupes américaines dans l'Aisne en juin 1918.

82 Strategic Simulations, Battle Cruiser, Strategic Simulations, 1987.

83 Le HMS Dreadnought, lancé en 1906, constitua une révolution dans la conception des cuirassés, avec l'introduction à la fois de la turbine à vapeur et d'une artillerie exclusivement constituée de pièces de gros calibre. Les bâtiments précédents, surclassés, furent en conséquence qualifiés de pré-dreadnoughts. En 1911, l'adjonction de canons supplémentaires augmenta encore la puissance d'une nouvelle génération, qualifiée alors de super-dreadnoughts.

84 Turcan Research Systems Limited, Dreadnoughts, Turcan Research Systems Limited, 1992. Peter Turcan, The Dreadnoughts Manual of Seamanship, Turcan Research Systems Limited, 1992, 93 p.

85 Ces trois marines correspondent en réalité à des scénarios prenant place pendant les guerres sino-japonaise (1894-1895) et russo-japonaise (1904-1905), toutes deux gagnées par le Japon. En conséquence, à cause de l'âge de ces navires ou des pertes pendant les affrontements (batailles de Yalou et de Tsushima en particulier), un grand nombre des bâtiments jouables (tous les chinois et une grande partie des russes notamment) n'ont pas connu la Première Guerre mondiale, et n'ont en conséquence pas été reportés dans les tableaux d'unités en annexe. De même et à l'inverse, beaucoup de bâtiments plus récents ayant eux participé à la Grande Guerre ne sont pas présents.

86 Nous n'avons pu nous procurer le manuel de ce jeu. Cependant, les critiques et capture 
revint sur ce sujet presque une décennie après Battle Cruiser avec Great Naval Battles V: Demise of the Dreadnoughts; 1914-18 $8^{87}$, reprenant les caractéristiques de son aîné en y ajoutant un aspect simulation. Cette recherche de précision toucha un peu plus tardivement et moins complètement les wargames à thématique terrestre, toujours à travers les titres de SSI : on citera surtout History Line: 1914-1918 ${ }^{88}$ en 1992, portage dans l'environnement de la Première Guerre mondiale d'un wargame futuriste paru l'année précédente, Battle Isle. Le gameplay général ne fut pas modifié, la plupart des scénarios supposant de mettre en ouvre des tactiques de mobilité anachroniques pour le front Ouest de la guerre, unique cadre géographique proposé. Cependant, une certaine attention au réalisme technique des unités pouvait être notée, à défaut d'un emploi tactique historiquement correct, et la documentation du jeu incluait un livret spécifiquement dédié à la description technique des différentes unités, avec une petite bibliographie. Si les unités d'infanterie, de cavalerie, d'artillerie et de marine étaient considérées de manière générique ${ }^{89}$, les principaux modèles de chars et avions étaient quant à eux présents et clairement différenciés. On notait même la présence d'automitrailleuses, équipements pourtant très peu connus du grand public ${ }^{90}$. SSI détailla toutefois réellement l'équipement terrestre en 1994 avec Wargame Construction Set II: Tanks!, dans lequel, si la Première Guerre mondiale (et surtout l'année 1918) ne représentait qu'une fraction du jeu, chacune des armées allemande, américaine, britannique et française n'en comportait pas moins une demi-douzaine de pièces d'artillerie différentes relativement bien identifiées, de même qu'un certain nombre de chars et équipements lourds d'infanteries ${ }^{91}$.

d'écran de celui-ci suggèrent un mode de jeu et une précision historique comparables à celle de Dreadnoughts. Software Sorcery, Jutland, Software Sorcery, 1993.

87 Divide By Zero, Great Naval Battles V: Demise of the Dreadnoughts; 1914-18, Strategic Simulations, 1996. Anonyme, Great Naval Battles V: Demise of the Dreadnoughts; 1914-18, Strategic Simulations, 1996, 130 p.

88 Blue Byte, History Line: 1914-1918, Blue Byte, Strategic Simulations, 1992. Anonyme, History Line: 1914-1918, Blue Byte, Strategic Simulations, 1992, 2 vol., $55+55$ p.

89 Par exemple, le jeu ne proposait que trois unités d'artillerie correspondant chacune à un grand type et non à un modèle précis : légère (jusqu’à $75 \mathrm{~mm}$ ), moyenne $(75 \mathrm{à} 150 \mathrm{~mm}$ ), et lourde (plus de $150 \mathrm{~mm}$ ). Dans la réalité, chacune de ces catégories regroupait un large éventail de pièces aux caractéristiques et usages bien différenciés. On notera tout de même la présence de sous-marins, ainsi que d'unités d'infanterie antichar correspond implicitement à des servants de fusil antichar Tankgewehr M1918.

90 De fait, la Charron modèle 1906 côté allié avait déjà était abandonnée depuis trois ans au moment de la déclaration de guerre (nous l'incluons tout de même dans le tableau synthétique en tant que très rare exemple de véhicule motorisé terrestre déjà obsolète en 1914), et une vingtaine seulement d'Ehrhardt E-V/4 allemandes ont été construites. Pour cette dernière, les créateurs du jeu s'appuyèrent sur une photographie et ne purent trouver ni le nom du modèle ni ses spécifications, se contentant donc de copier celles de la Charron adverse et de l'appeler Panzerspähwagen, la désignation des véhicules de reconnaissance allemands du second conflit mondial.

91 Strategic Simulations, Wargame Construction Set II: Tanks!, Strategic Simulations, 1994. 
Parallèlement au développement de ces wargames très documentés, la décennie 1990 a également vu à la fois une évolution dans la nature des jeux de stratégie appartenant aux genres préexistants, évoluant de la technicité réaliste vers la ludicité, et une ouverture sur la grande stratégie, ignorée jusque-là. Ainsi, dès 1986, World War I débutait la lignée des STR consacrés à la Grande Guerre, avec un mode de jeu stratégique et un mode tactique, à la manière des actuels Total War ${ }^{22}$. Celle-ci se poursuivit en 1990 avec Command $H Q$, un STR de grande stratégie dont un des quatre scénarios portait sur la Première Guerre mondiale ${ }^{93}$, et l'année suivante avec Powermonger ${ }^{94}$. Ce dernier était un jeu de stratégie et gestion en temps réel se déroulant dans un univers médiéval-fantastique et consistant à piller des villages à l'aide de fantassins pour conquérir des territoires; cependant une extension en proposa une version identique dans le cadre de la Grande Guerre, les unités combattant simplement à distance, sans plus de ressemblance avec la période en question. Ce genre disparut ensuite pendant une décennie, à l'exception de The War College: Universal Military Simulator 3 qui en 1995 se présentait à mi-chemin entre le wargame et le STR, avec un scénario sur la bataille de Tannenberg ${ }^{95}$. D'autre part, cité précédemment parmi les STR, Command HQ constituait également une nouveauté en ce qu'il portait la Première Guerre mondiale dans le champ des jeux de grande stratégie, en 1990. Deux ans plus tard, Scenario: Theatre of War y fut entièrement dédié ${ }^{96}$ : simpliste et sans prétention historique, à la manière d'un jeu de plateau de type Risk, il n'en mettait pas moins l'accent sur le rôle de l'industrie dans la conduite de la guerre, avec ses problématiques d'organisation, d'approvisionnement ou encore de grève des ouvriers. Le caractère systémique de ce type d'approche poussa en conséquence les concepteurs de jeux à tenter d'innover dans le fonctionnement de ceux-ci : en 1995, Fields of Battle ${ }^{97}$, quoique proche du précédent du point de vue du joueur, fut ainsi le premier jeu tous genres et sujets confondus ( $y$ compris hors Première Guerre mondiale) à utiliser une intelligence artificielle constituée de réseaux de neurones ${ }^{98}$. Comme les autres sous-genres de jeux de

92 Anonyme?, World War I, M.C. Lothlorien, 1986.

93 Ozark Softscape, Command HQ, Microplay Software, 1990.

94 Bullfrog Productions, Powermonger: World War I Edition, Electronic Arts, 1991.

95 Intergalactic Development Incorporated, The War College: Universal Military Simulator 3, GameTek (FL), 1995.

96 Starbyte Software, Scenario: Theatre of War, Starbyte Software, 1992.

97 BevelStone Production, Fields of Battle, BevelStone Production, 1995.

98 Un réseau de neurones correspond à un système d'intelligence artificielle inspirée du fonctionnement du cerveau biologique, et non fondé sur une programmation prédéterminée. Le réseau, dont les décisions sont le reflet de l'organisation interne, s'organise originellement de manière aléatoire et retient les configurations ayant donné les meilleurs résultats. Celles-ci sont ensuite utilisées comme socle pour une nouvelle " génération » de configurations, le cycle se répétant virtuellement à l'infini. Il s'agit donc, du moins en théorie, d'intelligences artificielles capables d'évoluer dans le sens d'une plus grande efficacité, soit d'" apprendre ". 
stratégie, SSI ne manqua pas de se positionner également sur la grande stratégie avec Imperialism en 1997, centré sur la géopolitique du XIX ${ }^{\mathrm{e}}$ siècle, mais s'achevant sur le déclenchement de la Grande Guerre ${ }^{99}$.

Ainsi, après des débuts centrés sur des wargames simples dans les années 1980, la stratégie vidéoludique à propos de la Grande Guerre s'est étendue à la fois à des simulations nettement plus complexes et détaillées, à des STR plus ludiques, et à la grande stratégie au début de la décennie 1990. Cette période sembla toutefois connaître un temps d'arrêt vers 1995, avec seulement deux sorties relativement anecdotiques peu après, lequel ne fut rompu qu'en 2000. Pendant ces quelques années de silence, le paysage des jeux de stratégie évolua radicalement, avec en particulier la sortie coup sur coup de Age of Empires (1997) et StarCraft (1998), qui consacrèrent pour un temps les STR jusqu'au milieu de la décennie suivante ${ }^{100}$. En toute logique, ces quelques années furent donc dominées par les STR dans le champ de la stratégie appliquée à la Première Guerre mondiale, avec des graphismes et un gameplay nettement améliorés par rapport à leurs prédécesseurs, bien que le réalisme historique n'ait pas été recherché : en effet, la caractéristique de ce type de jeux étant de permettre des mouvements et manœuvres rapides et précis, où la rapidité du joueur est mise à l'épreuve au même titre que sa réflexion, le principe même d'une guerre de position en est presque automatiquement exclu, et la Grande Guerre y était incluse plus pour son aspect " mythique " que pour une réelle volonté de simulation. En 2001, Empire Earth ${ }^{101}$ s'inspirait directement du gameplay d'Age of Empires en l'étendant à l'ensemble de l'histoire humaine, et en faisant correspondre une de ses quatorze ères ${ }^{102}$ à la Première Guerre mondiale bien qu'avec des unités génériques ${ }^{103}$. Plus notable encore, sur ces quatre campagnes, une était consacrée aux deux guerres mondiales jouées du point de vue allemand,

99 Frog City Software, Imperialism, Strategic Simulations, 1997.

100 Par la suite, et en lien avec le développement d'Internet, Defense of the Ancients (généralement abrégé en DotA, un scénario très spécifique pour Warcraft III: Reign of Chaos) en 2003 et World of Warcraft en 2004 orientèrent massivement les joueurs respectivement vers les MOBA (Multiplayer Online Battle Arena) et les MMORPG (Massively Multiplayer Online Role-Playing Game) dans la seconde moitié des années 2000.

101 Stainless Steel Studios, Empire Earth, Sierra On-Line, 2001.

102 Une "ère " désigne dans les STR une étape du jeu permettant au joueur de disposer d'un ensemble cohérent de bâtiments, unités et technologies, le passage à l'ère suivante requérant dans tous les cas un investissement (en ressources, temps, etc.) relativement conséquent. Dans les jeux historiques, les ères correspondent donc tout naturellement aux différentes étapes de développement des civilisations concernées.

103 Les types de certaines unités sont en réalité précisés, mais sont les mêmes quel que soit le pays (ou la "civilisation») joué, et correspondent en réalité à des rôles tactiques identiques d'une ère à l'autre, à la manière d'un pierre-papier-ciseaux : Sopwith (chasseur), Fokker (chasseurbombardier), Gotha (bombardier), Good Hope (frégate, historiquement un croiseur cuirassé de classe Drake), Dreadnought (cuirassé), Dardo (croiseur, historiquement un destroyer italien anachronique, entré en service en 1931), 57mm AT Gun (canon antichar, historiquement de la Seconde Guerre mondiale), A7V (char antichar), Mark V (char antipersonnel). 
avec sept scénarios, dont quatre consacrés au premier conflit et trois seulement au second. Il ne s'agissait pas moins avant tout de mettre en place des tactiques de guerre de mouvement, encore qu'un remarquable scénario sur la bataille de Verdun parvenait à rendre de manière particulièrement convaincante la difficulté d'effectuer la moindre avancée sur une ligne de front entièrement fortifiée. L'absence de STR récent consacré à la Première Guerre mondiale, laissant une opportunité dans un paysage vidéoludique historique de plus en plus exploré, amena par la suite au développement de The Entente: Battlefields World War I en 2004, spécifiquement dédié à la période, mais sans plus de réalisme au niveau du terrain (aucune carte ne comprend de tranchées) et du style de combat. On notait toutefois l'apparition remarquable de la Russie et de l'Autriche-Hongrie ${ }^{104}$, avec quelques armements clairement identifiés, en particulier le Sikorsky Ilya Muromets russe et le Leichter Kampfwagen II allemand, lequel n'a pourtant jamais dépassé le stade du prototype ${ }^{105}$. L'année suivante, World War I adapta à la Grande Guerre le moteur du jeu Blitzkrieg (2003), évidemment orienté vers la guerre de mouvement du second conflit mondial ${ }^{106}$. Le gameplay, s'il était un peu plus réaliste que celui de The Entente: Battlefields World War I avec la présence de quelques tranchées, n'en était donc pas moins totalement anachronique : des chars étaient ainsi disponibles dans un scénario sur l'invasion de la Belgique en 1914. Là encore, l'intérêt principal résidait dans le vaste éventail d'unités jouables, avec une emphase particulière portée sur les obusiers et mortiers lourds, mais également sur des véhicules peu connus (prototypes d'avant-guerre de chars ou d'automitrailleuses par exemple), incluant même des unités austro-hongroises et belges. Cette période du début des années 2000 marque nettement une importance nouvelle du STR par rapport au wargame tour par tour plus traditionnel, puisqu'un seul jeu de ce genre est sorti à cette époque, 1914: The Great War en 2002. Celui-ci semble avoir continué sur la lignée de ses prédécesseurs de la décennie 1990, avec des graphismes modernisés et une liste d'unités encore complétée ${ }^{107}$. Quant aux jeux de grande stratégie, la seule parution de l'époque fut Victoria: An Empire Under the Sun en 2003 (avec l'extension Victoria Revolution en 2006), qui à la manière d'Imperialism (1997), mais avec le moteur de jeu d'Europa Universalis (2000), se penchait sur les empires du XIX ${ }^{\mathrm{e}}$ siècle avec une conclusion vers $1920^{108}$. Un équivalent de la Grande Guerre n'y apparaissait donc que comme une éventualité potentielle et d'ailleurs peu rentable pour le joueur.

104 Lesta studio, The Entente: Battlefields World War I, Buka Entertainment, 2004.

105 Nous avons déduit les unités disponibles à partir des noms des fichiers de jeu d'une version de démonstration; il est donc possible voire probable que certaines unités disponibles dans le jeu final nous aient échappé.

106 Dark Fox, World War I, 1C Company, 2005.

107 Nous n'avons pu nous procurer le manuel de ce jeu, mais les critiques de l'époque font état d'une soixantaine d'unités différentes.

108 Paradox Interactive, Victoria: An Empire Under the Sun, PAN Vision, Strategy First, 2003. 
Un dernier ensemble de jeux a également pris place parallèlement aux jeux de stratégie (et aux simulateurs de vol omniprésents) entre le début des années 1990 et le milieu des années 2000, à savoir une douzaine de titres dans des genres variés et suivant une approche plus exploratoire : avec quasiment une sortie dans un nouveau genre chaque année, il s'agissait presque systématiquement d'unicums ou presque en leur temps, quelques-uns de ces genres n'ayant même jamais connu d'autre développement par la suite sur la thématique de la Première Guerre mondiale. Il s'agissait de manière générale de diverses variantes de jeux d'action, fondamentalement opposés au " sérieux » des jeux de stratégie, et cherchant plutôt à tirer parti des nouvelles possibilités offertes par les consoles de jeux. Pour certains, la Grande Guerre n'était en réalité qu'une toile de fond. C'est typiquement le cas du premier, The Young Indiana Jones Chronicles, sorti sur NES ${ }^{109}$ en $1993^{110}$ : adapté de la série télévisée du même nom, il s'agissait d'un jeu de plate-forme ${ }^{111}$ où le héros Indiana Jones suivait la trace d'un artefact égyptien à travers la révolution mexicaine, l'insurrection de Pâques à Dublin, une bataille de la Somme fantasmagorique ${ }^{112}$, un combat aérien sous forme de side scroller pour descendre le Baron Rouge, et un étrange quartier général allemand rempli de technologies " futuristes ». Le décalage était encore plus évident l'année suivante avec Castlevania: Bloodlines sur Sega Genesis ${ }^{113}$ : épisode d'une série de jeux mettant en scène un chasseur de vampires affrontant les forces du comte Dracula, celui-ci présentait la Grande Guerre comme un sacrifice vampirique et consistait à affronter des squelettes réanimés de soldats allemands dans plusieurs lieux européens, dont seuls une fabrique de munitions allemande et le château de Versailles avaient un quelconque lien avec la guerre (tout en restant de simples prétextes de décors pour de classiques niveaux de plate-forme). En 1996 et 1997, ce sont deux jeux d'aventure en point-and-click ${ }^{114}$ qui firent référence aux mouvements nationalistes serbes ayant précipité le début de la guerre : dans Titanic: Adventure Out of Time, le héros avait la possibilité sur le Titanic de modifier l'histoire en empêchant les deux guerres mondiales (notamment en s'emparant de diamants

109 Nintendo Entertainment System, console commercialisée par Nintendo de 1985 à 1995 aux États-Unis.

110 Jaleco, The Young Indiana Jones Chronicles, Jaleco USA, 1993.

111 Type de jeu où le personnage contrôlé par le héros avance dans un niveau constitué d'une succession de plateformes en éliminant des ennemis génériques jusqu’à affronter un boss de fin de niveau nettement plus difficile à battre.

112 On y trouve, pêle-mêle et sans cohérence, tous les éléments stéréotypiques du front occidental de la Grande Guerre : allemands à casques à pointe, triplans Fokker, chars primitifs, automitrailleuses, boue, obus, motocyclettes, grenades, gaz, casemates, barbelés, Grosse Bertha, Zeppelins, le tout disposé selon les seuls codes du jeu de plate-forme.

113 Konami, Castlevania: Bloodlines, Konami, 1994.

114 Type de gameplay consistant pour le joueur, voyant l'action sous forme d'instantanés fixes, à cliquer sur des éléments de décor, des personnages, des objets ou des choix de dialogue pour faire avancer l'intrigue. 
destinés à financer la Main Noire $\left.{ }^{115}\right)^{116}$, et on retrouvait les mêmes réseaux terroristes serbes impliqués dans des trafics d'armes à bord de l'Orient-Express dans The Last Express ${ }^{17}$. En 2002, plus dynamique, car abandonnant le pointand-click pour l'action, Eternal Darkness: Sanity's Requiem sur GameCube s'inspirait directement de l'univers de Howard Phillips Lovecraft ${ }^{118}$, pour proposer une aventure d'horreur psychologique se déroulant dans treize tableaux différents entre 2000 avant et 2000 après notre ère, l'un d'eux étant la cathédrale d'Amiens transformée en hôpital militaire en $1916^{119}$. Enfin et toujours en lien avec le fantastique, Shadow Hearts: Covenant sur PlayStation 2 en 2004 suivait les codes typiques des RPG ${ }^{120}$ japonais pour chasser un démon du village de Domrémy ${ }^{121}$ assiégé par les Allemands en $1915^{122}$. Par ailleurs, en 1999, la Grande Guerre, ou plutôt une uchronie prenant place à Petrograd en 1917 (avec des armes en grande partie anachroniques), faisait son apparition dans le champ des FPS avec Codename Eagle ${ }^{123}$ : jeu d'aventure et d'infiltration, celui-ci disposait surtout d'un mode multijoueur qui évoque manifestement, avec un peu d'avance, les jeux de type Battlefield ${ }^{124}$. L'autre FPS de la période, Iron Storm paru en 2002, était lui aussi une uchronie, cette fois dans une Première Guerre mondiale qui aurait perduré jusqu'en 1964 et où les alliés occidentaux s'opposeraient à un bloc constitué de l'Allemagne, la Russie et la Chine ${ }^{125}$ : l'environnement était globalement inspiré de la Grande Guerre, mais avec des armes pour la grande majorité liée au second conflit mondial voire à la guerre froide. Nettement plus orienté arcade et humour encore, typique de la PlayStation, Hogs of War mélangeait en 2000 un peu de tactique à de l'arcade pure, dans une Grande Guerre à peine esquissée combattue par des cochons ${ }^{126}$. L'année suivante, Wardoves: Secret Weapon of World War I consistait même au sens propre en un tir aux pigeons, puisqu'il s'agissait

115 Société secrète nationaliste serbe fondée en 1911, supervisant les actions anti-autrichiennes en Serbie avant-guerre et ayant notamment fourni les armes employées par Gavrilo Princip et ses complices lors de l'attentat de Sarajevo en 1914.

116 CyberFlix, Titanic: Adventure Out of Time, GTE Entertainment, Europress, 1996.

117 Smoking Car Productions, The Last Express, Brøderbund, 1997.

118 Cet univers, développé à travers de nombreuses nouvelles dans les années 1920 et 1930, est dans son ensemble désigné sous le nom de "mythe de Cthulhu ».

119 Silicon Knights, Eternal Darkness: Sanity's Requiem, Nintendo, 2002.

120 Role Playing Game, genre de jeu dans lequel le joueur incarne directement un personnage, le caractère et les habilités de celui-ci évoluant au cours du jeu.

121 Le choix de ce lieu n'a rien d'aléatoire : Domrémy-la-Pucelle est le village natal de Jeanne d'Arc, élément culturel auquel les joueurs japonais sont susceptibles de pouvoir se référer.

122 Nautilus, Shadow Hearts: Covenant, Midway Home Entertainment, 2004.

123 Refraction Games, Codename Eagle, Take-Two Interactive, 1999.

124 La ressemblance avec les premiers Battlefield n'est pas fortuite : Refraction Games, le studio ayant produit Codename Eagle, a ensuite très rapidement été intégré à DICE (qui produit les Battlefield), et le moteur de jeu a été réemployé d'un titre à l'autre.

125 4X Studios, Iron Storm, Wanadoo, Dreamcatcher Interactive, Reef Interactive, 2002.

126 Infrogrames, Hogs of War, Infogrames Sheffield House, 2000. 
simplement d'éliminer des pigeons voyageurs envahissant l'écran, en évitant les avions, chars et grenades lancées sur le joueur ${ }^{127}$. Il faut enfin mentionner un ultime jeu de cette période, le seul parmi ces jeux d'action ou d'aventure à réellement se pencher sur la Grande Guerre en tant que telle : WWI Medic, produit et édité par Bay 12 Games en $2004^{128}$. Ce studio, uniquement composé des frères Tarn et Zach Adams, est surtout célèbre pour Dwarf Fortress (2006), jeu de gestion médiéval-fantastique en génération procédurale réputé à la fois pour son niveau de détails, sa difficulté, sa complexité, l'intelligence du concept et ses graphismes en caractère ASCII ${ }^{129}$. Sans surprise, un certain nombre de ces caractéristiques se retrouvaient dans WWI Medic: dans des graphismes simplistes volontairement inspirés de ceux de la décennie 1980, le joueur contrôlait un médecin devant aller chercher et soigner des blessés dans le no man's land entre deux assauts et au milieu des bombardements, le gameplay étant extrêmement limité, mais parvenant tout de même à rendre tangibles le chaos du champ de bataille et la dangerosité du rôle de brancardier.

\section{Action, casual gaming et jeux amateurs : 2005-2020}

Nous avons pu constater que, jusqu'au milieu des années 2000, les jeux consacrés à la Première Guerre mondiale (simulateurs de vol exceptés) correspondaient majoritairement à des jeux de stratégie (principalement wargames dans les années 1980-1990, plutôt STR au début des années 2000, avec quelques jeux de grande simulation), avec un certain nombre d'autres genres apparus ponctuellement depuis le début des années 1990, mais jamais exploités de manière soutenue. Après un certain creux en 2005-2006 (un seul jeu paru pour chacune de ces années hors simulateurs de vol), les sorties se sont fait beaucoup plus soutenues depuis 2007, avec au minimum 3 jeux hors simulateurs quasiment chaque année, et jusqu'à une dizaine en 2015. De plus, les genres plébiscités étaient totalement différents : un écroulement des wargames et STR au profit de la grande stratégie, une emphase importante sur les jeux d'aventure, les FPS voire les jeux d'horreur, et le développement de jeux plus orientés vers le casual gaming ${ }^{130}$, ainsi que de jeux produits par des amateurs et diffusés sur la plate-forme Steam.

127 Redfire Software, Wardoves: Secret Weapon of World War I, uWish Games, 2001.

128 Bay 12 Games, WW1 Medic, Bay 12 Games, 2004.

129 American Standard Code for Information Interchange. Norme de codage de caractères limitée mais extrêmement répandue, permettant d'afficher les lettres, les chiffres et quelques caractères spéciaux ou de ponctuation. Pour Dwarf Fortress, il s'agit dans l'absolu d'un abus de langage car la table des caractères employée est la Codepage 437, un peu plus étendue.

130 Pratique de jeux simples à prendre en main et avec une difficulté modérée, permettant un jeu "facile ", au contraire des jeux plus traditionnels qu'il est nécessaire d'apprendre à maitriser. 
Comme indiqué précédemment, les jeux de stratégie sur la Grande Guerre ont donc été depuis une dizaine d'années totalement dominés par la grande stratégie, avec quasiment un titre par an dans ce genre. Pour se renouveler et se démarquer de la concurrence, ces différents jeux portant à quelques exceptions près toujours sur le même sujet travaillèrent généralement sur une amélioration et multiplication des aspects liés à la gestion, sur un agrandissement des cartes, et/ou sur une augmentation de leur précision (sans toutefois atteindre celle d'un wargame dans tous les cas ${ }^{131}$ ). Le premier jeu de grande stratégie de cette "série ", Aggression: Europe Under Fire en 2007, se rattachait toutefois encore à un modèle préexistant, avec une juxtaposition de phases de grande tactique et de stratégie en STR, à la manière des Total War ou de ce qui avait déjà été tenté par World War I en $1986^{132}$. La période chronologique englobait cependant les deux conflits mondiaux, avec plus d'emphase sur le second, et ce mode de jeu ne fut pas poursuivi par la suite. L'année suivante, le jeune studio AGEod, spécialisé dans les jeux de grande stratégie, revenait à une présentation plus classique avec World War One: La Grande Guerre ${ }^{133}$. L'insistance était mise sur les détails de la carte et la possibilité de jouer un nombre important de nations, y compris des pays européens " oubliés " peu assimilés à la Grande Guerre (Grèce, Roumanie...), mais également des espaces colonisés (sous différentes modalités) par les grandes puissances en guerre, en Afrique ou au Moyen-Orient par exemple. En 2010 et 2011, Victoria II puis Darkest Hour: A Hearts of Iron Game, tous deux édités par Paradox Interactive, présentèrent une configuration similaire, bien que la Première Guerre mondiale ne représentait qu'une fraction de ces jeux : comme son homonyme de 2003, le premier couvrait avant tout le XIX ${ }^{\mathrm{e}}$ siècle ${ }^{134}$, et le second ne consacrait qu'un seul scénario à cette guerre, les autres se tournant vers la Seconde ${ }^{135}$. Très rapidement, les jeux entièrement dédiés à la Grande Guerre s'orientèrent vers une carte plus détaillée, mais en conséquence recentrée sur l'Europe (en conservant tout de même l'Afrique du Nord, le Proche-Orient et l'Atlantique) et non plus mondiale : ce fut le cas de Strategic Command Classic: WWI, aux graphismes assez simplistes, en $2011^{136}$, et Commander: The Great War en $2012^{137}$, tous

131 Schématiquement et sous réserve de quelques exceptions, les jeux de grande stratégie les plus précis individualisent des chef-lieux de départements et des unités de l'ordre de la brigade (un peu moins de 10000 hommes) ; les wargames, pour leur part, détaillent au moins les chef-lieux de cantons, et des unités de l'ordre de la compagnie (moins de 300 hommes).

132 Lesta Studio, Aggression: Europe Under Fire, Buka Limited, 2007.

133 Le studio AGEod est grenoblois, ce qui peut expliquer le choix d'un sous-titre en français. AGEod, World War One: La Grande Guerre, Ascaron Entertainment, 2008.

134 Paradox Development Studio, Victoria II, Paradox Interactive, 1C/Snowball Studios, 2010.

135 Darkest Hour Team, Darkest Hour: A Hearts of Iron Game, Paradox Interactive, 2011.

136 Fury Software, Matrix Games, Strategic Command Classic: WWI, Matrix Games, 2011.

137 The Lordz Games Studio, Slitherine, Matrix Games, Commander: The Great War, Slitherine, Matrix Games, 2012. 
deux édités par Matrix Games et par Slitherine pour le second. Dès 2014, afin de poursuivre cette recherche d'améliorations, le studio AGEod édité par Slitherine proposa de nouveau une carte plus grande dans To End All Wars, les "régions » (ou zones différenciées) se comptant en milliers, avec également un développement des aspects annexes au champ de bataille, comme la personnalité des dirigeants militaires et civils, les technologies, la logistique ou encore le moral ${ }^{138}$. L'année suivante, Making History: The Great War mettait lui aussi l'accent sur le commerce et les infrastructures, à une échelle mondiale ${ }^{139}$. Plus récemment encore, on vit enfin réapparaître le choix de ne traiter en détail qu'une situation chronogéographique bien précise, en l'occurrence la Russie. AGEod et Slitherine se consacrèrent ainsi à la révolution russe dans Revolution Under Siege en $2015^{140}$, et en 2018 la campagne de Tannenberg voisinait deux campagnes napoléoniennes et deux sur les offensives soviétiques de 1945 dans Wars Across The World: Russian Battles ${ }^{141}$.

Il convient également de mentionner l'existence d'un sous-genre plus inattendu, le STR de grande stratégie multijoueur, expérimenté dès 2009 avec succès sur navigateur, avec Supremacy 1914 $1^{142}$, et de nouveau employé dans Supreme Ruler: The Great War en 2017, celui-ci s'orientant plus vers un jeu libre, permettant de jouer la plupart des pays du monde sur le plan de leur gestion interne et non uniquement des opérations militaires, et ce potentiellement à travers tout le $\mathrm{XX}^{\mathrm{e}}$ siècle, même si les parties commençaient systématiquement pendant la Grande Guerre ${ }^{143}$. Les autres genres de jeux de stratégie, sous-représentés, sont réapparus très tardivement : en 2015 pour les wargames avec Spirit of War, extrêmement similaire à l'antique History Line: 1914-1918 de $1992^{144}$, suivi par le très documenté The Operational Art of War IV en 2017, à la limite du jeu de grande stratégie en termes d'échelle et faisant un usage du code OTAN tout à fait classique dans la lignée des premiers wargames, mais dont seule une vingtaine de scénarios sur les $200 \mathrm{du}$ jeu concernait la Grande Guerre (dont des conflits périphériques, à savoir les deux guerres balkaniques de 1912 et 1913, la guerre soviéto-polonaise de 1919-1921, et la guerre grécoturque de 1919-1922) ${ }^{145}$. Un unique STR est à relever sur la même période, prenant enfin en compte les spécificités de la Première Guerre mondiale. Avant d'être proposé comme jeu individualisé en 2015, Battle of Empires: 1914-1918

138 AGEod, To End All Wars, Slitherine, 2014.

139 Muzzy Lane Software, Factus Games, Making History: The Great War, Factus Games, 2015.

140 Sep Reds, AGEod, Revolution Under Siege, Slitherine, 2015.

141 Strategiae, Wars Across The World: Russian Battles, Plug In Digital, 2018.

142 Bytro Labs, Supremacy 1914, Bytro Labs, 2009.

143 BattleGoat Studios, Supreme Ruler: The Great War, BattleGoat Studios, 2017.

144 Nous n'avons pu accéder au manuel de ce jeu, mais il pourrait même s'agir au sens propre d'un remake de History Line: 1914-1918. G-OLD, Spirit of War, Plug In Digital, 2015.

145 TrickeySoft LLC, The Operational Art of War IV, Slitherine, 2017. 
avait été originellement développé comme un mod pour Men of War: Assault Squad (2011), axé sur la Seconde Guerre mondiale ${ }^{146}$; le moteur de celui-ci était toutefois suffisamment polyvalent (prenant notamment en compte le fait que des combats de position ont également eu lieu pendant la Seconde Guerre mondiale) pour pouvoir être adapté à un autre type de conflit de manière pertinente. Il s'agissait d'un STR joué à un niveau tactique, où chaque soldat pouvait être contrôlé individuellement, et dans des environnements historiquement réalistes. La diversité et la précision des armements, quoique n'atteignant pas celle d'un wargame, pouvaient être soulignées, y compris pour les armes individuelles ou encore les automitrailleuses. Les unités incluaient également des catégories souvent oubliées, comme les armées austro-hongroise ou ottomane ${ }^{147}$, les troupes de l'ANZAC dans l'armée britannique, les partisans russes, ou encore l'infanterie belge (jouable dans l'armée française). Il conviendrait d'ajouter Steam Squad paru en $2016^{148}$, au gameplay similaire, mais dans une Première Guerre mondiale uchronique à l'ambiance steampunk ${ }^{149}$.

De nouveaux types de jeux assimilables à ceux de stratégie se sont par ailleurs développés de manière importante depuis le début des années 2000, à commencer par ceux que nous regroupons sous le nom de castle attack, que l'on pourrait considérer comme des jeux de tactique simplifiés. Il s'agit de croisements entre des STR très simples et des jeux d'artillerie de type Crush the Castle (2009) ou Angry Birds (2009), consistant à détruire un " château " adverse et ses occupants en ajustant le tir d'une machine de siège. Dans ces jeux de castle attack, chaque camp dispose d'une base, qu'il peut éventuellement avoir conçue lui-même, doit gérer ses ressources pour recruter des unités (de distance ou de contact), et envoyer celles-ci à l'attaque de manière coordonnée au moment opportun pour capturer la base adverse. On comprend donc que le gameplay de ces jeux se concentre avant tout sur la gestion du temps, mais n'exploite pas les trois dimensions spatiales, les unités allant généralement en ligne relativement droite d'une base à l'autre. Ceci facilite

146 Great War Team, Battle of Empires: 1914-1918, Best Way Soft, 2015.

147 Nous n'avons pu trouver une liste complète des unités que pour la première version du jeu, alors qu'il n'était encore qu'un mod et n'incluait pas encore l'Empire ottoman, et pour l'armée russe dans le jeu final. Nous ne pouvons donc préciser la liste des unités ottomanes jouables, et celles des autres armées (excepté la russe) sont probablement quelque peu différentes dans le jeu définitif.

148 Bretwalda Games, Steam Squad, Bretwalda Games, 2016.

149 Sous-genre particulièrement courant de rétrofuturisme, soit d'univers uchroniques mélangeant éléments archaïques et futuristes par rapport à une époque donnée. Le steampunk, plus précisément, se présente généralement comme un début de $\mathrm{XX}^{\mathrm{e}}$ siècle alternatif dans lequel les technologies propres au XIX ${ }^{\mathrm{e}}$ siècle (et en particulier le moteur à vapeur, d'où son nom) auraient été améliorées et miniaturisées, en lieu et place du développement d'autres branches techniques (comme le moteur à explosion). L'aspect esthétique correspond à un mélange exacerbé d'éléments victoriens et industriels. 
la programmation et la jouabilité de ces titres, qui peuvent ainsi souvent être considérés comme des casual games, mais en font également un genre particulièrement intéressant pour la description d'une guerre de position. En effet, si l'on fait abstraction de l'aviation qui obéit à d'autres logiques, et de la guerre des mines (globalement quasiment absente de tous les jeux de notre corpus), les combats de tranchées du front occidental de la Grande Guerre consistaient principalement pour l'infanterie à sortir de ses tranchées et progresser en ligne droite vers les tranchées adverses, en bonne coordination avec l'artillerie. Ce potentiel a été très bien saisi dès 2008 avec Warfare 1917, jeu sur navigateur dans lequel le joueur doit capturer la tranchée adverse à l'aide de forces principalement composées d'infanterie, avec un soutien d'artillerie ${ }^{150}$. Si la durée de vie du jeu reste courte, les mitrailleuses et barrages d'artillerie adverses réduisent régulièrement les vagues d'attaques du joueur à néant avant que celles-ci ne soient même à porter de tirer, débouchant naturellement sur une compréhension du rôle des bombardements préliminaires et donc des mécanismes basiques de la guerre de tranchées. Le potentiel en casual gaming étant évident, une quasi-copie pour iOS est parue dès l'année suivante, Trenches ${ }^{151}$, suivie de Trenches 2 en $2011^{152}$, puis en 2016 à nouveau sur PC avec Command of War, ajoutant quelques éléments de gameplay (un aspect shooter notamment ${ }^{153}$ ) sur un principe toujours identique ${ }^{154}$. En 2018, deux jeux développèrent encore cette idée en y ajoutant des aspects s'approchant plus des STR tactiques: On The Western Front, où le joueur plaçait lui-même les tranchées (ainsi que les sapes de mines, éléments suffisamment rares pour être relevés) ${ }^{155}$ et Soldiers Lost Forever (1914-1918), semblables aux précédents, mais où le joueur contrôlait de plus un soldat en particulier ${ }^{156}$. On peut assimiler à ces jeux ceux de tower defense, dans lesquels le joueur doit cette fois empêcher des vagues d'ennemis de capturer sa base, en plaçant les défenses appropriées à des emplacements prédéfinis. Ce genre a été adapté à la Grande Guerre avec Toy Soldiers en $2010^{157}$ et Toy Soldiers: War Chest en $2015^{158}$, avec des mitrailleuses et canons en guise de "tours ", et dédramatisant l'ambiance en plaçant le décor dans un coffre à jouets au milieu d'une chambre d'enfant. Pourtant, le principe même du gameplay - le massacre à la chaîne de masses

150 ConArtist, Warfare 1917, Armor Games, 2008.

151 Thunder Game Works, Trenches, Thunder Game Works, 2009.

152 Thunder Game Works, Trenches 2, EA Games, 2011.

153 Le terme de shooter, employé seul, désigne un genre de jeu consistant à détruire des ennemis simplement en cliquant dessus, sans autre forme de stratégie.

154 Il semble que ce jeu soit resté à l'état de version bêta jouable. Red Cube Games, Command of War, Red Cube Games, 2016.

155 Aggroblakh, On The Western Front, Aggroblakh, 2018.

156 Jake Olson, Soldiers Lost Forever (1914-1918), Impartial Studios, 2018.

157 Signal Studios, Toy Soldiers, Microsoft Game Studios, 2010.

158 Signal Studios, Toy Soldiers: War Chest, Ubisoft, 2015. 
de fantassins indifférenciés - procurait en réalité un sentiment de pertinence historique étrangement efficace pour un tower defense, d'autant que le joueur pouvait lui-même commander ses " tours ", et que celles-ci représentaient des armements réels bien identifiés (d'ailleurs parfois peu communs, par exemple pour l'artillerie antiaérienne) $)^{159}$.

Tirant parti de l'amélioration des capacités des machines en matière de graphisme, le dernier genre de jeu plus ou moins réaliste, parmi ceux axés sur le combat, à s'être développé de manière importante depuis le milieu des années 2010, est celui des FPS et simulateurs assimilables (outre les simulateurs de vol). On notera avant cette date, en 2009, la sortie à la fois de Darkest of Days, relativement réaliste pour son temps, mais dont la bataille de Tannenberg ne constituait qu'un des multiples tableaux historiques ${ }^{160}$, et de Boğaz Harbi: Çanakkale Savaşı Destanı, remarquablement consacré à la bataille de Gallipoli envisagée du point de vue ottoman, mais à la diffusion limitée étant donnée sa concentration sur le marché turcophone ${ }^{161}$. Ce genre se développa toutefois soudainement en 2015 avec Verdun ${ }^{162}$ : il s'agissait cette fois d'un jeu multijoueur entièrement consacré au front occidental, toujours pratiqué, avec des combats de tranchée se voulant réalistes (dans le mode de jeu le plus historique, chaque équipe doit s'emparer de la tranchée adverse ou défendre la sienne) et des armes bien détaillées. La difficulté de la visée (sans assistance et avec une stabilité imparfaite rendant compte du poids de l'arme) et les temps de rechargement importants y sont rendus, avec de plus l'inclusion des grenades ou encore des armes de corps-à-corps, qu'il s'agisse de couteaux, de pelles ou de masses. L'absence de bombardement important et la limitation du nombre de joueurs (techniquement, afin d'équilibrer le gameplay sur chaque carte, et humainement puisque le jeu reste relativement peu connu, surtout par rapport à Battlefied $1^{163}$ ) rendent toutefois difficile une immersion dans un combat violent et soutenu, la situation de jeu corres-

159 Nous n'avons pas pu nous procurer la liste complète des unités disponibles, il manque en conséquence dans les relevés celles de l'armée française ainsi que les avions.

160 8monkey Labs, Darkest of Days, Phantom EFX, 2009.

$161 \mathrm{Il}$ semble que le jeu n'ait pas dépassé la version bêta, et nous n'avons pas pu trouver de liste des armes représentées. Kodgraf Oyun Stüdyosu, Boğaz Harbi: Çanakkale Savaşı Destanı, Kodgraf Oyun Stüdyosu, 2009.

162 M2H, Blackmill Games, Verdun, M2H, Blackmill Games, 2015.

163 D'après les statistiques publiées par la plate-forme Steam, le nombre moyen de joueurs par jour sur un mois pour Verdun a dépassé 650 en juin 2017, mais il n’a jamais atteint les 200 entre mars et octobre 2018. En comparaison, Battlefield 2, sorti en 2005 soit une décennie auparavant, rassemble toujours actuellement deux fois plus de joueurs. Battlefield 1 rassemble à la mi-2018 autour de 100000 joueurs par jour en moyenne, et les jeux les plus pratiqués peuvent aisément dépasser le million. Il est à noter que la série Battlefield comprend à l'heure actuelle six titres, Battlefield 2 étant le second comme son numéro l'indique, mais Battlefield 1 est le cinquième (le 1 fait référence à la Première Guerre mondiale). 
pondant plutôt à des escarmouches. En 2017, la même équipe développait Tannenberg, équivalent de Verdun pour le front oriental (prenant également en compte l'armée autrichienne) et détaillant encore plus les différents armements individuels, mais le jeu rencontre encore moitié moins de public que son prédécesseur déjà peu fréquenté ${ }^{164}$. On peut ajouter Revolt 1917 en 2018 , jeu indépendant situant son action au sein de la révolte arabe et prenant en compte les spécificités du Proche- et Moyen-Orient en termes de gestion de l'hydratation pour le personnage ${ }^{165}$, ainsi que The Trench 1916, toujours en développement, se situant pendant la bataille de Verdun avec un gameplay semble-t-il proche de celui de Verdun (2015), mais non multijoueur, permettant ainsi potentiellement de mieux rendre l'ambiance générale en ajoutant des personnages contrôlés par l'ordinateur ${ }^{166}$.

Cependant, le FPS incontournable sur la Première Guerre mondiale en termes de répercussion médiatique et de nombre de joueurs est très nettement Battlefield 1, transportant dans la Grande Guerre un gameplay multijoueur traditionnellement orienté arcade et très dynamique, développé sur une série centrée autour de la Seconde Guerre mondiale et des guerres modernes voire futuristes ${ }^{167}$. En conséquence, bien que le jeu propose des cartes en lien avec la guerre de tranchées, celles-ci ne sont pas réellement utilisées comme telles avec un rôle protecteur statique. Ceci est notamment valable pour le mode de jeu multijoueur, le moins historique, mais le plus pratiqué, le jeu " solo " présentant des scénarios nettement plus ancrés dans la réalité quoique toujours orientés arcade. Par contre, à la différence de Verdun (2015) ou Tannenberg (2017), la popularité du jeu permet de mobiliser un grand nombre de joueurs par partie, évitant l'effet de carte vide. Le grand intérêt de Battlefield 1 réside toutefois dans la variété de cartes, figurant avec un réalisme relativement travaillé tant les fronts européens, dont ceux peu connus comme les Alpes, que l'Orient, mais aussi d'armes disponibles, en particulier pour l'armement individuel, bien que souvent quelque peu simplifiées (leur maniement n'est pas particulièrement réaliste et les temps de rechargement sont écourtés, par exemple). Les armes disponibles devant être similaires dans toutes les armées et permettre une importante fluidité du gameplay, ce jeu a souvent été décrié par la critique comme mettant en scène un armement anachronique. De fait, celui-ci met l'accent sur les armes à tir rapide, qu'il s'agisse des fusils-mitrailleurs (ou mitrailleuses) effectivement en usage à l'époque, mais aussi des armes portatives que sont les pistolets mitrailleurs et fusils automatiques. Pour ces dernières (souvent

164 Tannenberg n'a jamais dépassé une moyenne mensuelle de 200 joueurs par jour, et n'a à l'heure actuelle (octobre 2018) pas atteint la centaine depuis décembre 2017. M2H, Blackmill Games, Tannenberg, M2H, Blackmill Games, 2017.

165 Juhbee, Revolt 1917, Juhbee, 2018.

166 Gallica Game Studio, The Trench 1916, Gallica Game Studio, à paraitre.

167 EA DICE, Battlefield 1, Electronic Arts, 2016. 
regroupées sous le terme générique de " mitraillettes »), les modèles présentés sont soit des prototypes qui n'ont jamais vu le combat, soit des armes ayant réellement été employées, mais en très petit nombre et de manière anecdotique, pour des troupes de choc ou de façon expérimentale. Si la prédilection des joueurs (orientée par le gameplay) pour ces armes en multijoueur a tendance à transformer ces curiosités historiquement rarissimes en armes de premier choix dans le jeu, il faut tout de même remarquer que la diversité en armes offerte par Battlefield 1 ne s'arrête pas là : un grand nombre de modèles de revolvers et pistolets ont été modélisés, de même qu'une sélection très convenable de fusils et grenades, plusieurs fusils à pompe (particulièrement liés à l'armée américaine pendant la guerre), ou encore des armes blanches incluant la pelle, divers couteaux, mais aussi des armes improvisées. On y trouve également un choix appréciable de canons et avions, mais surtout des véhicules terrestres incluant, outre les chars habituels, des automitrailleuses pour certaines jamais encore incluses dans un jeu sur la Grande Guerre ainsi que des motocyclettes. Enfin, Battlefield 1 a fait polémique à sa sortie car il permettait de jouer des soldats américains, mais non français ou russes, qui n’ont été ajoutés que par la suite. Si ce choix est critiquable et manifestement lié à des raisons commerciales, idéologiques et politiques, il faut tout de même remarquer qu'il s'agit également du premier titre à rendre jouable les États-Unis parmi les FPS et genres assimilés (à l'exception de Wargame Construction Set II: Tanks! en 1994, depuis longtemps oublié, et de Jutland (2006), limité à la guerre navale), ainsi que l'Italie. Malgré ces choix surprenants et l'historicité douteuse de certaines armes et du gameplay, il faut ainsi reconnaître à Battlefield 1 une capacité d'immersion du joueur et de choix de matériel seulement concurrencée, dans certaines limitées, par Verdun (2015) et Tannenberg (2017), avec un engouement populaire ayant permis de faire redécouvrir cette guerre à de nombreux joueurs.

Parallèlement, cette période a également vu la parution de quelques titres de simulation navale ou assimilés. En 2006, Jutland, supplément pour le jeu Distant Guns sur la guerre russo-japonaise et ayant connu de multiples additions dans les années suivantes, reprenait la logique de Dreadnoughts (1992) et Jutland (1993) avec une simulation extrêmement détaillée et réaliste, notamment sur la gestion des paramètres liés à la distance entre les navires, qui en fait toujours une référence plus d'une décennie après ${ }^{168}$. La liste des bâtiments présentés avait encore été agrandie, notamment par l'adjonction des marines française et américaine. Même pour les nationalités déjà simulées auparavant, la liste des cuirassés et croiseurs représentés était presque aussi complète, mais il fallait y ajouter un important travail sur les destroyers et l'intégration de type de bâtiments jusqu'alors négligés, comme les porte-avions, mouilleurs de mines, croiseurs auxiliaires, sous-marins, quelques monitors britanniques 
employés comme artillerie côtière, et une liste appréciable de dirigeables allemands. On notera simplement que, le jeu étant centré autour de la bataille du Jutland en 1916, les navires trop anciens à cette époque n'étaient pas représentés, tout comme ceux lancés après. Il faut ajouter à ce jeu 1914 Shells of Fury paru l'année suivante, simulant le fonctionnement d'un sous-marin allemand attaquant des convois dans l'Atlantique du point de vue du capitaine, avec une vue au périscope à la première personne ${ }^{169}$. La diversité présentée était toutefois limitée, avec seulement quatre types de sous-marins jouables, dont deux génériques ${ }^{170}$. Malgré la diversité et qualité des simulateurs de sousmarins consacrés à la Seconde Guerre mondiale ou à la guerre froide (la série des Silent Hunter pour ne citer qu'elle), la Première Guerre mondiale est donc restée une période oubliée pour cette arme, le plus récent U-Boats en 2016 étant avant tout un shooter éloigné de toute réelle simulation ${ }^{171}$.

Pour les bâtiments de surface, cette lignée ponctuelle presque uniquement constituée de simulateurs très précis et complets connut quant à elle un équivalent de Battlefield 1, à savoir un jeu plus dynamique et arcade destiné à un public plus large, mais tout de même un minimum documenté avec un nombre appréciable de bâtiments historiques, avec World of Warships en $2015^{172}$. La série des World of, qui comprend également des opus sur les chars (World of Tanks en 2009 ${ }^{173}$ ) et sur les avions (World of Warplanes en 2013), se penche avant tout sur la Seconde Guerre mondiale avec ses prémisses pendant l'entre-deux-guerres et ses suites au début de la guerre froide, et n'inclut en conséquence aucun avion et quasiment aucun char de la Grande Guerre à de très rares exceptions près ${ }^{174}$. Son concurrent direct, War Thunder (2013), en fait également totalement abstraction. Cependant, afin d'offrir une évolution aussi nette dans les armements que ses équivalents sur terre et sur mer, World of Warships inclut des navires remontant jusqu'au début du $\mathrm{XX}^{\mathrm{e}}$ siècle. Il ne s'agit pas de simulations de batailles historiques, mais uniquement d'affrontements entre équipes de joueurs, avec une prise en compte minimale, voire inexistante, du fonctionnement des navires. Cependant, cette extension temporelle permet de jouer quelques bâtiments de chaque grande classe et de

169 Rondomedia, h2f Informationssysteme, 1914 Shells of Fury, Strategy First, 2007.

170 Anonyme, 1914. Shells of Fury, Strategy First, 2007, 28 p.

171 Astra Game Studio, U-Boats, Astra Game Studio, 2016.

172 Wargaming.net, Lesta Studio, World of Warships, Wargaming.net, Lesta Studio, 2015.

173 Wargaming Minsk, World of Tanks, Wargaming.net, 2009.

174 A savoir le Renault FT, qui a été employé jusqu’à la Seconde Guerre mondiale, et une variante de celui-ci, ainsi que le Mark I et la Lanchester $4 \times 2$ Armoured Car intégrés uniquement pour un mode de jeu célébrant le centenaire du premier emploi des chars en 2016. Ces jeux sont en constante évolution et de nouvelles unités sont ajoutées régulièrement ; les principales nationalités ayant pris part à la Première Guerre mondiale étant déjà présentes, ces ajouts sont toutefois peu susceptibles de concerner des armes de la Grande Guerre, qui constituent au mieux le tout premier niveau du jeu. 
l'époque de la Grande Guerre pour les marines britannique et allemande, un important choix de cuirassés français et américains, ainsi que des bâtiments japonais et russes plus récents que ceux de la guerre russo-japonaise traités longtemps auparavant dans Dreadnoughts (1992). L'ensemble représente tout de même une cinquantaine de navires (dont un certain nombre de projets n'ayant jamais vu le jour, choix étrange compte tenu du nombre de bâtiments ayant réellement existé, et pour lequel les éditeurs sont régulièrement critiqués), soit plus d'un sixième des bâtiments du jeu. On notera enfin, pour revenir aux chars et clore cette partie sur les simulations, le développement en cours du jeu indépendant Cry of War, tourné vers la simulation de combats de chars pendant la Grande Guerre, une situation historiquement très rare ${ }^{175}$.

Comme évoqué précédemment, depuis le milieu des années 2000 se sont également développés d'autres genres de jeux, qui n'avaient jusqu'alors été que très ponctuellement abordés. En particulier, un grand nombre de jeux d'aventure ou assimilés a été proposé depuis 2007, prenant la Grande Guerre comme cadre pour le développement d'une intrigue plus ou moins liée. Ce format permet en particulier de produire des jeux avec des moyens limités, mais principalement intéressants pour leur histoire. Le premier d'entre eux, Cover Front paru en 2007, était typiquement un point-and-click gratuit sur navigateur, le joueur contrôlant une espionne devant infiltrer le manoir d'un scientifique malfaisant pendant une Première Guerre mondiale alternative $^{176}$. Cet ensemble point-and-click-puzzles-espionnage fit d'ailleurs recette puisque dès les deux années suivantes parurent deux jeux de ce type consacrés à Mata Hari, le premier consistant à infiltrer une base de sous-marins allemands ${ }^{177}$, et le second se plaçant dans le contexte de la Belle Époque et de l'escalade vers le conflit ${ }^{178}$ (soit dans les deux cas des activités sans rapport avec la carrière d'espionne - quasi-inexistante - de Mata Hari). Après quelques années sans jeu d'aventure, ceux-ci refirent leur apparition en 2014, au moins en partie en lien avec les commémorations du Centenaire de la guerre. En France, Ubisoft publia Soldats Inconnus : Mémoires de la Grande Guerre, mélangeant principalement point-and-click et puzzles pour suivre le destin de plusieurs personnages impliqués dans les combats du front occidental (des soldats français, allemand et américain, et une infirmière), dans un environnement voulu évocateur et revendiqué comme éducatif ${ }^{179}$. On notera à son sujet la présence comme personnage jouable d'un chien de recherche

175 L'identification des unités jouables dans le tableau synthétique s’appuie uniquement sur les images publiées du développement. ShanghaiWindy, Cry of War, ShanghaiWindy, à paraître.

176 Pastel Games, Covert Front, 2007.

177 Frogwares Game Development Studio, Secret Missions: Mata Hari and the Kaiser's Submarines, Frogwares Game Development Studio, 2008.

178 Cranberry Production, Mata Hari, Viva Media, 2009.

179 Ubisoft Montpellier, Soldats inconnus : Mémoires de la Grande Guerre, Ubisoft, 2014. 
de blessés, attention intéressante portée au rôle des animaux dans ce conflit, que Battlefield 1 (2016) a également inclus en proposant une "simulation " de pigeon voyageur. La même année, beaucoup plus restreint, mais également nettement plus réaliste, fut publié Our World War: Interactive Episode, un épisode interactif d'une série de la BBC plaçant le joueur dans le rôle d'un caporal devant commander un groupe de soldats durant les combats de High Wood, pendant la bataille de la Somme ${ }^{180}$.

Toujours en 2014, il faut signaler Fritz, un projet indépendant proche du jeu de rôle, dont le financement participatif a échoué et qui fut donc annulé, mais dont les premières images laissèrent présager un jeu réellement complet concernant l'évocation de la vie des soldats ${ }^{181}$. Il s'agissait de diriger un appelé allemand tentant de survivre à la vie quotidienne dans les tranchées, sans être accusé de couardise, alternant quelques rares et intenses moments de combat, avec des phases de jeu principales d'attente, voire d'ennui, trompées par l'humour noir, la socialisation avec les autres soldats, ou la recherche de ressources. Dans les années suivantes, les jeux d'aventure prirent un tournant plus contemplatif, jusqu'à devenir plutôt des jeux d'exploration dans certains cas. En 2015, To Burn in Memory se présentait comme une découverte sous forme de textes, à la manière d'un livre, d'une cité ruinée allégorique faisant entre autres référence à la Grande Guerre, mais sans connexion directe avec celle-ci ${ }^{182}$. L'année suivante, Tales from the Void, incluant des phases de combat tactique en temps réel, envoyait l'équipage d'un sous-marin britannique perdu en mer dans une dimension parallèle peuplée d'extraterrestres, les marins démunis devant parvenir à rentrer chez eux ${ }^{183}$. Plus récemment encore, Because Wére Here - Mohnblume und Blauerose proposait sous forme de visual novel ${ }^{184}$ une histoire d'amour dans le contexte de la guerre de tranchées ${ }^{185}$, et Temporality, nettement plus expérimental, faisait observer au joueur une scène de charge en vue de côté, lui permettant uniquement de faire avancer et reculer le temps sur une musique introspective, la démarche étant présentée comme une invitation à la réflexion ${ }^{186}$. Sur une thématique proche, Within

$180 \mathrm{Mi}$, Our World War: Interactive Episode, BBC, 2014.

181 Truceful Entertainment, Fritz, Truceful Entertainment, 2014.

182 Orihaus, To Burn in Memory, Orihaus, 2015.

183 Ce sous-marin, le HMS E18, avait réellement été perdu dans des circonstances mystérieuses au large de l'Estonie en 1916, la découverte de son épave suggérant en 2009 qu'il avait simplement heurté une mine sous-marine. PortaPlay, Tales from the Void, Black Dog, 2016.

184 Genre, particulièrement répandu au Japon, s'approchant d'un « livre dont vous êtes le héros » : le joueur joue un personnage interagissant avec d'autres, chaque étape du récit étant représentée sous forme d'un tableau avec le personnage en interaction et une boîte de dialogue, le joueur étant amené de temps à autre à prendre des décisions quant à la conduite du dialogue et à influencer le déroulement du jeu.

185 Studio Elfriede, Because We're Here - Mohnblume und Blauerose-, Studio Elfriede, 2018.

$186 \mathrm{Ce}$ jeu n'est pas encore disponible à l'heure où nous écrivons, nous nous appuyons donc sur 
Whispers: The Fall, actuellement en développement, fait incarner au joueur une jeune femme s'entretenant avec les fantômes de soldats morts pour tenter de retrouver son frère disparu ${ }^{187}$.

Parallèlement, s'est également développé sur la même période un certain nombre de jeux d'horreur (au sens fantastique du terme), que celle-ci soit utilisée comme un moyen pour décrire la violence et la mort des combats de la Grande Guerre, ou, plus souvent, qu'elle soit considérée comme un cadre approprié pour situer une aventure d'horreur ; il était fréquent que le gameplay en lui-même se rapproche des jeux d'action ou des FPS. Ainsi, en 2007, The Darkness ne portait pas fondamentalement sur la Grande Guerre, mais, dans le cadre d'un scénario d'horreur fantastique, le personnage du XXI siècle se trouvait pour certaines phases du jeu propulsé dans un no man's land surréaliste et dépourvu de sens, affrontant des soldats vivants ou morts et les Cavaliers de l'Apocalypse tout en cherchant son ancêtre combattant ${ }^{188}$. Le plus célèbre de ces jeux est paru en 2009 : NecroVisio $N^{189}$, avec une suite l'année suivante ${ }^{190}$. Il s'agit techniquement d'un FPS où le joueur combat à la fois des soldats vivants, d'autres morts et réanimés, ainsi que des démons, le tout dans des tranchées, des forteresses, des laboratoires rétrofuturistes ou encore les Enfers. La violence et l'absurdité de la mort dans les tranchées servent clairement de fondement à l'ambiance générale, contrebalancée par les commentaires du personnage principal versant dans l'humour noir et clairement inspirés de Duke Nukem 3D (1996). On pourra aussi remarquer que le joueur combat en partie avec des armes historiques, dont le rechargement peut être étrangement réaliste pour un jeu orienté arcade.

En 2011, 1916 - Der Unbekannte Krieg, développé par une équipe d'étudiants, se rapprochait plus volontiers de l'aspect psychologique du jeu d'horreur $^{191}$ : le joueur y était perdu dans un labyrinthe de tranchées, poursuivi par des dinosaures, cherchant quelques maigres indices (volontairement en allemand afin de désorienter encore plus le joueur, le jeu étant uniquement proposé en anglais et en danois) pour tenter de trouver l'unique échelle de sortie. Il s'agissait manifestement d'une évocation de la folie susceptible de saisir les soldats, le protagoniste se faisant d'ailleurs tuer par un obus sitôt qu'il avait gravi l'échelle. Le jeu Ad Infinitum, encore en développement en septembre 2017 et probablement abandonné depuis, aurait pu présen-

les présentations et les images de développement publiées. James Earl Cox III, Temporality, James Earl Cox III, 2018.

187 Thorium Studios, Within Whispers: The Fall, Thorium Studios, à paraître en 2019.

188 Starbreeze Studios, The Darkness, 2K Games, 2007.

189 The Farm 51, NecroVisioN, 1C Company, 505 Games, Aspyr Media, 2009.

190 The Farm 51, NecroVisioN, 1C Company, 2010.

191 Kriegsgraben und Stormvogel, 1916-Der Unbekannte Krieg, 2011. 
ter un concept semblable ${ }^{192}$. Autre projet étudiant de la même année 2011, The Snowfield faisait diriger au joueur un soldat perdu dans une tempête de neige après une bataille ${ }^{193}$; il s'agissait avant tout d'un essai concernant une narration non programmée à l'avance et presque improvisée, mais le tout rendait tout de même une ambiance extrêmement sombre et désespérée. L'année suivante, le genre de l'horreur fut appliqué à la thématique du jeu de rôle mâtiné de tactique au tour par tour, avec Call of Cthulhu: The Wasted Land $^{194}$; il s'agissait de l'univers de Lovecraft, déjà rencontré en 2002 avec Eternal Darkness: Sanity's Requiem, lequel était en effet globalement contemporain de la Grande Guerre. Au milieu des combats dans un décor de no man's land, un groupe d'investigateurs et de soldats y enquêtait sur la présence d'une divinité malfaisante tout en combattant morts-vivants et démons, un scénario typique de cet univers. Enfin, entre 2014 et 2017, a été publiée une série de jeux d'action voire d'arcade, à commencer par le shooter indépendant Super Trench Attack!, mettant en scène un Rambo des tranchées dans un gameplay humoristique simple et efficace ${ }^{195}$. Dans plusieurs styles différents, on peut ainsi citer les shoots 'em up ${ }^{196}{ }^{\text {Don'Yoku }}{ }^{197}$ (proche du manic shooter ${ }^{198}$ ), et 1917 - The Alien Invasion $D X^{199}$, sans aucun fond historique et seulement vaguement inspirés de la guerre d'un point de vue graphique, ainsi que Great War 1914, un Retro FPS ${ }^{200}$ transposant dans l'univers de la Grande Guerre le gameplay de Wolfenstein 3D (1992) ${ }^{201}$. Enfin, on signalera une mission du jeu Assassin's Creed Syndicate (situé globalement au XIX ${ }^{\mathrm{e}}$ siècle) se déroulant dans le Londres de 1916 pendant un bombardement de Zeppelin, incluant des phases d'infiltration typiques de cette franchise ainsi qu'un passage d'action consistant à manier un canon antiaérien ${ }^{202}$.

192 StrixLab, Ad Infinitum, StrixLab, à paraître (?).

193 Singapore-MIT Gambit Game Lab, The Snowfield, Singapore-MIT Gambit Game Lab, 2011.

194 Red Wasp Design, Call of Cthulhu: The Wasted Land, Red Wasp Design, 2012.

195 Retro Army, Super Trench Attack!, Retro Army, 2014.

196 Jeu de tir dans lequel le joueur contrôle typiquement un vaisseau situé en bas de l'écran, et doit détruire des vagues d'ennemis arrivant par le haut de l'écran en évitant leurs projectiles. L'archétype de ce genre est Space Invaders (1978).

197 Dark-Spot Studio, Don'Yoku, Dark-Spot Studio, 2015.

198 Sous-genre de shoot 'em up particulièrement difficile, dans lequel l'écran est saturé de projectiles et le joueur n'a une marge de manœuvre que de quelques pixels. On peut citer particulièrement Touhou Project (1996).

199 OtakuMaker.com, 1917 - The Alien Invasion DX, Andrade Games, 2016.

200 Le terme " Retro " précédant un genre qui désigne un jeu récent employant volontairement le gameplay et les graphismes de jeux anciens, typiquement des années 1980 et 1990.

201 Musrka, Great War 1914, Blue Whale Games, 2017.

202 Ubisoft Quebec, Assassin's Creed Syndicate, Ubisoft, 2015. 


\section{Conclusion}

La Première Guerre mondiale apparaît comme un conflit difficile à restituer dans les jeux vidéo : les combats de tranchées, perçus à tort comme la constante de ce conflit, imposent une confrontation longue et statique, voire immobile, provoquant un ennui subi par les combattants l'ayant réellement vécue, mais qui n’a rien de vendeur pour un jeu vidéo. De fait, les aspects de gameplay intéressants que peut fournir un contexte guerrier, si l'on excepte l'action pure, sont le fait de pouvoir manœuvrer, se déplacer pour contourner ou piéger l'ennemi ; en somme le mouvement. En conséquence, cette démarche de restitution est en fait restée délicate et minoritaire en dehors des simulateurs de vol, du fait qu’il s'agissait d'un conflit de position, et ce malgré un nombre de titres parus depuis la fin des années 1970 en réalité plutôt élevé. Dans les jeux d'avions centrés sur la simulation de vol ou les duels aériens, et ce quel que soit le genre précis de chaque jeu, le gameplay fait appel à l'indépendance du joueur et lui donne des choix, qui se justifient par l'intérêt porté spécifiquement au matériel plus qu’à un scénario - aspects que l'on retrouve quelque peu dans des jeux de simulation consacrés aux matériels terrestres et maritimes, et à la prise d'initiative possible en tant que duelliste. Par contre, les wargames et autres STR, qui semblent être des genres évidents pour décrire une guerre mondiale et effectivement très développés pour la Seconde Guerre mondiale, ont été moins mis à profil et presque systématiquement en corrompant la réalité du combat pour y inclure une guerre de mouvement anachronique - la référence à la Grande Guerre étant essentiellement conservée comme élément surprenant pour accrocher l'attention du joueur potentiel. Par ailleurs, la Première Guerre mondiale est une thématique qui se prête bien aux jeux d'arcade depuis le début des années 1980, ainsi quaux jeux d'horreur comme allégorie des horreurs de la guerre, mais toujours avec un rapport éloigné à la réalité historique.

Au niveau du matériel mis en scène, celui-ci est rarement très détaillé et la grande majorité des jeux se focalisait sur un ou deux modèles connus du public (parfois par analogie avec ceux de la Seconde Guerre mondiale, quand ils n'ont pas réellement servi dans les deux conflits et donc bénéficié de la notoriété du second), complétant éventuellement avec des engins choisis plus aléatoirement. Ce cas est particulièrement criant pour l'artillerie (Tableau 3) dans laquelle, sur 67 pièces représentées, ne se retrouvent régulièrement que le $7,7 \mathrm{~cm}$ Feldkanone 16 et le 10,5 cm leichte Feldhaubitze 16 allemands, le Canon de $75 \mathrm{~mm}$ modèle 1897 français et le Ordnance QF 18-pounder britannique. Les choix sont encore plus éparpillés pour l'armement individuel (Tableau 2) : les armes ne sont représentées qu'une ou deux fois (généralement dans Verdun, Tannenberg ou Battlefield 1), et, sur 140 modèles, seuls le fusil Mauser (Gewehr 98), la mitrailleuse Maxim (Maschinengewehr 08/15) et le pistolet Luger (Lange Pistole 08), tous allemands, sont relativement courants. Par le faible nombre de modèles en service à l'époque et la fasci- 
nation exercée par ceux-ci sur le public, les véhicules blindés sont par contre représentés de manière plus exhaustive (Tableau 4) : pour les trois grandes nations du front Ouest, deux modèles de char sont quasi omniprésents, à savoir le Sturmpanzerwagen A7V pour l'Allemagne (seul char produit par cette nation durant le conflit), le Char Renault FT et le Saint-Chamond pour la France, le Mark I et le Mark A Whippet pour la Grande-Bretagne ; même les automitrailleuses pourtant moins connues, y compris les russes, sont régulièrement mises en scène. Le constat est similaire pour les avions, avec une représentation assez diversifiée qui ne masque pas la prédominance au moins aussi écrasante de quelques types "légendaires » (Tableau 5) : pour l'Allemagne les Albatros D.III et Fokker D.VII, mais surtout le Fokker Dr.I dans la livrée rouge personnelle de Manfred von Richthofen ; les Spad S.VII et S.XIII français ; et pour la Grande-Bretagne les Airco DH.2 de Havilland, Sopwith Pup, Royal Aircraft Factory S.E. 5, et surtout, sorte de nemesis du Baron Rouge, le Sopwith Camel. Le cas des navires est lui plus particulier, car dominé par des jeux exhaustifs s'adressant à un public hautement connaisseur (Tableau 6) : Dreadnoughts, Great Naval Battles V: Demise of the Dreadnoughts; 1914-18 et Jutland proposent ainsi des listes de bâtiments quasi complètes et donc en grande partie similaires, principalement différenciées par les légers décalages chronogéographiques concernant les contextes traités.

Il apparaît en conséquence que les parutions récentes et médiatisées, en particulier Soldats Inconnus : Mémoires de la Grande Guerre, Battlefield 1 et dans une moindre mesure Verdun, s'ils ne sont pas aussi uniques dans leur sujet que ce qui est généralement avancé - souvent présentés comme des exceptions, ils ne représentent en réalité que $2 \%$ du corpus étudié ici - explorent par contre des modes de jeu relativement nouveaux sur le sujet de la Grande Guerre, et à destination d'un public plus large que la grande majorité des titres. La parution d'un jeu " grand public " adoptant une approche réellement historique, prenant en compte toutes les nations en conflit, avec une simulation réaliste - y compris concernant les aspects les plus humains de la bataille, comme la mort, la faim ou la folie - et faisant fi des éléments iconiques de l'imaginaire populaire, mais anecdotiques dans la guerre - comme l'omniprésent triplan rouge du Baron Rouge - reste cependant toujours à désirer, et pourrait possiblement présenter un intérêt pédagogique, certes brutal, voire choquant, par le réalisme encore très rarement exploré.

\section{Cyril Lacheze et Marion Weckerle}

Université Paris 1 Panthéon-Sorbonne Institut d'Histoire Moderne et Contemporaine Équipe d'Histoire des Techniques cyril.lacheze@univ-paris1.fr marion.weckerle@etu.univ-paris1.fr 
Cyril Lacheze fait porter ses recherches sur les modes de conception d'éléments historiques envisagés sous leur aspect d'objets techniques, l'amenant à croiser des sources de natures variées. La restitution de ceux-ci, comme source ou comme élément de médiation, intègre cette réflexion et inclut pleinement la possibilité de modélisation par le jeu.

Université Paris 1 Panthéon-Sorbonne - Institut d'Histoire Moderne et Contemporaine Équipe d'Histoire des Techniques

- Lacheze Cyril, «Pour une analyse systémique de la technique : exemple de la production de terre cuite architecturale ", Cahiers François Viète, III, 6, 2019, p. 77-107, en ligne.

- Lacheze Cyril, Weckerle Marion, «Apport de l'archéologie à l'étude des opérations militaires modernes et contemporaines ", in Drevillon Hervé, Guillemin Dominique (éds), Histoire des opérations militaires. Sources, objets, méthodes, Vincennes, Service Historique de la Défense, 2018, p. 199-209.

Marion Weckerle travaille sur les processus d'invention et d'innovation dans le domaine de l'hydraviation, dans une approche systémique. Sa période d'étude inclut la Première Guerre mondiale, et s'intéresse également à la perception ancienne et actuelle de cette aéronautique, incluant son apparition dans les jeux vidéo. Elle bénéficie en 2018 d'une allocation de thèse du ministère des armées.

Université Paris 1 Panthéon-Sorbonne - Institut d'Histoire Moderne et Contemporaine Équipe d'Histoire des Techniques

- Weckerle Marion, "L'hydravion dans les revues sportives françaises, 1906-1914 ", p. 19, Bulletin des doctorants du centre de recherche en histoire du XIX siècle, $\mathrm{n}^{\circ} 7$, printemps 2018, p. 153-169, en ligne.

- Weckerle Marion, «L'hydraviation allemande et austro-hongroise dans la Première Guerre mondiale ", e-Phaïstos, vol. 4, n 2, octobre 2015, 8 p., en ligne.

\section{Résumé}

La sortie récente de quelques jeux vidéo particulièrement médiatisés en lien avec les commémorations du Centenaire de la Première Guerre mondiale a induit dans la conscience collective une impression de nouveauté de cette thématique dans le champ vidéoludique. En réalité, celle-ci a été abondamment, mais inégalement traitée depuis les années 1980, dans environ 150 titres. Nous parcourons leur historique afin d'en restituer les modalités d'approche du conflit proposées, depuis les premiers simulateurs de vol jusqu'aux jeux de stratégie réalistes, en passant par l'arcade ou l'horreur.

\section{Mots-clés}

histoire, simulateur de vol, stratégie, tactique, arcade, Première Guerre mondiale, jeu.

\section{Abstract}

The recent release of some video games particularly publicized in connection with the commemorations of the centenary of the First World War induced in the collective consciousness an impression of novelty of this thematic in the field of video games. In fact, it has been treated extensively but unevenly since the 1980s, in about 150 games. We go through the history of these in order to highlight the approaches of the conflict, from the first flight simulators to realistic strategy games, including arcade or horror.

\section{Keywords}

history, fight simulator, strategy, tactic, arcade, First World War, game. 


\section{TABLEAU 1}

Liste des jeux analysés. Ce corpus regroupe la totalité des jeux vidéos concernant en totalité ou en partie, sous une forme non modée, la Première Guerre mondiale, sous réserve de quelques oublis. Les genres donnés sont simplifiés, de nombreux titres pouvant être classés dans plusieurs catégories. Concernant les jeux marqués comme à paraitre, les informations fournies sont celles connues en octobre 2018.

\begin{tabular}{|c|c|c|c|c|}
\hline Année & Titre & Développeur & Éditeur & Genre \\
\hline 1975 & BiPlane & Fun Games & Fun Games & Side scroller ${ }^{1}$ \\
\hline 1976 & BiPlane 4 & Fun Games & Fun Games & Side scroller \\
\hline 1976 & Wings & Electra Game & Electra Game & Side scroller \\
\hline 1980 & Red Baron & Atari & Atari & Simulateur de vol \\
\hline 1983 & Air Battle & $?$ & Philips Export B.V. & Side scroller \\
\hline 1983 & Battle 1917 & Mark Lucas & $\begin{array}{l}\text { Cases Computer } \\
\text { Simulations }\end{array}$ & Wargame \\
\hline 1983 & Blue Max & Synapse Software & $\begin{array}{l}\text { Synapse Software ; } \\
\text { U.S. Gold }\end{array}$ & Side scroller \\
\hline 1983 & Eagles & Paul Murray & Strategic Simulations & Wargame \\
\hline 1983 & Flying Ace & Microcomputer Games & $\begin{array}{l}\text { The Avalon Hill } \\
\text { Game Company }\end{array}$ & Side scroller \\
\hline 1983 & Snoopy and the Red Baron & Atari & Atari & Side scroller \\
\hline 1984 & Sopwith & BMB Compuscience & BMB Compuscience & Side scroller \\
\hline 1985 & Red Baron & B. D. Hambüchen & Labochrome & Side scroller \\
\hline 1985 & Sky Kid & Namco & Namco ; Sunsoft & Side scroller \\
\hline 1985 & Sopwith ${ }^{2}$ & BMB Compuscience & BMB Compuscience & Side scroller \\
\hline 1986 & Gallipoli & A. et O. Bishop & $\begin{array}{l}\text { Cases Computer } \\
\text { Simulations }\end{array}$ & Wargame \\
\hline 1986 & The Great War 1914 & Marc Summerlott & D.K.G. & Wargame \\
\hline 1986 & World War I & ? & M.C. Lothlorien & RTS \\
\hline 1987 & Battle Cruiser & Strategic Simulations & Strategic Simulations & Wargame naval \\
\hline 1987 & $\begin{array}{l}\text { Halls of Monteruma: A Battle History } \\
\text { of the United States Marine Corps }\end{array}$ & Strategic Studies Group & $\begin{array}{l}\text { Strategic Studies } \\
\text { Group }\end{array}$ & Wargame \\
\hline 1988 & Grand Fleet & Simulations Canada & Simulations Canada & Wargame naval \\
\hline 1989 & War Eagles & Cosmi Corporation & Cosmi Corporation & Simulateur de vol \\
\hline 1990 & Blue Max: Aces of the Great War & $\begin{array}{l}\text { Artech Digital } \\
\text { Entertainment }\end{array}$ & Three-Sixty Pacific & Simulateur de vol \\
\hline 1990 & Command HQ & Ozark Softscape & Microplay Software & RTS \\
\hline
\end{tabular}

1. Tous les jeux de type side scroller répertoriés ont pour thématique l'aviation.

2. Ce jeu, parfois désigné sous le nom de Sopwith 2, est une seconde version du titre éponyme paru l'année précédente, y ajoutant un certain nombre de fonctionnalités. Une édition "définitive" a été publiée en 2000 sous le nom de Sopwith: The Author's Edition. 


\begin{tabular}{|c|c|c|c|c|}
\hline 1990 & Dogfight & Richard Ling & Amiga (?) & Side scroller \\
\hline 1990 & Chocks Away & Andrew Hutchings & $\begin{array}{l}\text { The Fourth } \\
\text { Dimension }\end{array}$ & Simulateur de vol \\
\hline 1990 & Red Baron ${ }^{3}$ & Dynamix & Sierra On-Line & Simulateur de vol \\
\hline 1990 & Wings ${ }^{4}$ & Cinemaware & Cinemaware & Simulateur de vol \\
\hline 1991 & Knights of the Sky & MicroProse & MicroProse & Simulateur de vol \\
\hline 1991 & Powermonger: World War I Edition & Bullfrog Productions & Electronic Arts & RTS \\
\hline 1991 & Warbirds & Atari & Atari & Simulateur de vol \\
\hline 1992 & Blue Baron & Steven Metcalf & $\begin{array}{l}\text { Zeppelin Games } \\
\text { Limited }\end{array}$ & Side scroller \\
\hline 1992 & Dreadnoughts & $\begin{array}{l}\text { Turcan Research } \\
\text { Systems Limited }\end{array}$ & $\begin{array}{l}\text { Turcan Research } \\
\text { Systems Limited }\end{array}$ & Simulation maritime \\
\hline 1992 & History Line: $1914-19185$ & Blue Byte & $\begin{array}{l}\text { Blue Byte ; Strategic } \\
\text { Simulations }\end{array}$ & Wargame \\
\hline 1992 & Scenario: Theatre of War & Starbyte Software & Starbyte Software & Grande stratégie \\
\hline 1992 & Wings 2: Aces High ${ }^{6}$ & Malibu Interactive & Namco & Simulateur de vol \\
\hline 1993 & Air Duel: 80 Years of Dogfighting & Vektor Grafix & MicroProse & Simulateur de vol \\
\hline 1993 & Jutland & Software Sorcery & Software Sorcery & Simulation maritime \\
\hline 1993 & The Ancient Art of War in the Skies & Evryware & MicroProse & $\begin{array}{l}\text { Tactique / } \\
\text { simulateur de vol }\end{array}$ \\
\hline 1993 & The Young Indiana Jones Chronicles & Jaleco & Jaleco USA & Action \\
\hline 1994 & Castlevania: Bloodlines & Konami & Konami & Plate-forme \\
\hline 1994 & Dawn Patrol & Rowan Software & Empire Interactive & Simulateur de vol \\
\hline 1994 & Wargame Construction Set II: Tanks! & Strategic Simulations & Strategic Simulations & Wargame \\
\hline 19947 & Wings of Glory & Origin Systems & Electronic Arts & Simulateur de vol \\
\hline 1995 & Dawn Patrol: Head to Head & Rowan Software & $\begin{array}{l}\text { Empire Interactive } \\
\text { Entertainment }\end{array}$ & Simulateur de vol \\
\hline 1995 & Fields of Battle & BevelStone Production & $\begin{array}{l}\text { BevelStone } \\
\text { Production }\end{array}$ & Grande stratégie \\
\hline 1995 & $\begin{array}{c}\text { The War College: Universal Military } \\
\text { Simulator } 3\end{array}$ & $\begin{array}{l}\text { Intergalactic } \\
\text { Development }\end{array}$ & GameTek (FL) & Wargame \\
\hline
\end{tabular}

3. Un éditeur de scénario est venu s'y adjoindre deux en plus tard sous le nom de Red Baron: Mission Builder (1992).

4. Une version de ce jeu remasterisée par le studio Hippo Entertainment, entièrement en 3D, est parue en 2014.

5. Ce jeu est également connu sous son nom américain : The Great War: 1914-1918.

6. Ce jeu est également connu sous son nom européen : Blazing Skies.

7. Une seconde version est parue l'année suivante, avec une fluidité et des graphismes améliorés. 


\begin{tabular}{|c|c|c|c|c|}
\hline & & Incorporated & & \\
\hline 1996 & $\begin{array}{c}\text { Great Naval Battles V: Demise of the } \\
\text { Dreadnoughts; 1914-18 }\end{array}$ & Divide by Zero & Strategic Simulations & Maritime \\
\hline $1996^{8}$ & Fyying Corps & Rowan Software & Empire Interactive & Simulateur de vol \\
\hline 1996 & Titanic: Adventure Out of Time & CyberFlix & $\begin{array}{l}\text { GTE Entertainment ; } \\
\text { Europress }\end{array}$ & Aventure \\
\hline 1997 & $A C M 1918$ & Aqua Pacific & $\begin{array}{l}\text { Project Two } \\
\text { Interactive BV }\end{array}$ & Simulateur de vol \\
\hline 1997 & Imperialism & Frog City Software & Strategic Simulations & Grande stratégie \\
\hline 1997 & Red Baron II & Dynamix & Sierra On-Line & Simulateur de vol \\
\hline 1997 & The Last Express & $\begin{array}{l}\text { Smoking Car } \\
\text { Productions }\end{array}$ & $\begin{array}{l}\text { Brøderbund; } \\
\text { Interplay; } \\
\text { Entertainment } \\
\text { DotEmu }\end{array}$ & Aventure \\
\hline 1997 & Wing Nuts: Battle in the Sky & Rocket Science Games & $\begin{array}{l}\text { BMG Interactive } \\
\text { Entertainment }\end{array}$ & Simulateur de vol \\
\hline 1998 & Crash Bandicoot 3: Warped & Naughty Dog & $\begin{array}{l}\text { Sony Computer } \\
\text { Entertainment }\end{array}$ & Arcade \\
\hline 1998 & Red Baron 3-D9 & Dynamix & Sierra On-Line & Simulateur de vol \\
\hline 1999 & Codename Eagle & Refraction Games & $\begin{array}{c}\text { Take-Two } \\
\text { Interactive ; Tsukuda } \\
\text { Original }\end{array}$ & FPS \\
\hline 1999 & Curse You! Red Baron & Dynamix & Sierra On-Line & Simulateur de vol \\
\hline 2000 & Aces High & HiTech Creations & HiTech Creations & Simulateur de vol \\
\hline 2000 & Hogs of War & Infogrames & $\begin{array}{c}\text { Infogrames Sheffield } \\
\text { House }\end{array}$ & Stratégie \\
\hline 2000 & Master of the Skies: The Red Ace $e^{10}$ & Fiendish Games & $\begin{array}{c}\text { Global Star } \\
\text { Software ; Small } \\
\text { Rockets }\end{array}$ & Simulateur de vol \\
\hline 2001 & Empire Earth & Stainless Steel Studios & Sierra On-Line & RTS \\
\hline 2002 & Red Ace Squadron ${ }^{11}$ & Small Rockets & Global Star Software & Simulateur de vol \\
\hline 2001 & $\begin{array}{c}\text { Wardoves: Secret Weapon of World War } \\
I\end{array}$ & Redfire Software & uWish Games & Shooter \\
\hline 2002 & 1914: The Great War & $\begin{array}{l}\text { TriNodE Entertainment } \\
\text { Systems }\end{array}$ & $\begin{array}{l}\text { JoWooD Productions } \\
\text { Software AG }\end{array}$ & Wargame \\
\hline
\end{tabular}

8. Une édition Gold est parue l'année suivante, incluant des améliorations à plusieurs niveaux et ajoutant le Fokker D-VII aux appareils jouables.

9. Ce jeu peut être considéré comme une version améliorée de Red Baron II, paru l’année précédente.

10. Également connu sous le titre de Red Ace.

11. Il s'agit d'un contenu additionnel par rapport au Red Ace paru l'année précédente (cf. note précédente). 


\begin{tabular}{|c|c|c|c|c|}
\hline 2002 & Eternal Darkness: Sanity's Requiem & Silicon Knights & Nintendo & $\begin{array}{c}\text { Action-aventure } \\
\text { horreur }\end{array}$ \\
\hline 2002 & Iron Storm & 4X Studios & $\begin{array}{c}\text { Wanadoo } \\
\text {; Dreamcatcher } \\
\text { Interactive ; Reef } \\
\text { Interactive }\end{array}$ & FPS \\
\hline 2003 & Victoria: An Empire Under the Sun & Paradox Interactive & $\begin{array}{l}\text { PAN Vision / } \\
\text { Strategy First }\end{array}$ & Grande stratégie \\
\hline 2003 & Wings of Honour & CITY interactive & CITY interactive & Simulateur de vol \\
\hline 2004 & Shadow Hearts: Covenant & Nautilus & $\begin{array}{l}\text { Midway Home } \\
\text { Entertainment }\end{array}$ & RPG \\
\hline 2004 & The Entente: Battlefields World War $I^{12}$ & Lesta studio & Buka Entertainment & RTS \\
\hline 2004 & Wings of War & Silver Wish Games & $\begin{array}{l}\text { Gathering of } \\
\text { Developers }\end{array}$ & Simulateur de vol \\
\hline 2004 & WWI Medic & Bay 12 Games & Bay 12 Games & Action \\
\hline 2005 & Red Baron & $\begin{array}{c}\text { Atomic Planet } \\
\text { Entertainment Limited }\end{array}$ & Davilex Games B.V. & Simulateur de vol \\
\hline 2005 & World War I & Dark Fox & 1C Company & RTS \\
\hline 2006 & First Eagles: The Great War 1918 & Third Wire Productions & G2 Games & Simulateur de vol \\
\hline 2006 & Flyboys Squadron & iEntertainment Network & $\begin{array}{l}\text { iEntertainment } \\
\text { Network }\end{array}$ & Simulateur de vol \\
\hline 2006 & Jutland & Norm Koger ; Jim Rose & Storm Eagle Studios & Simulateur maritime \\
\hline 2006 & Sky Aces & $\begin{array}{l}\text { The X Studio } \\
\text { Productions }\end{array}$ & IncaGold & Simulateur de vol \\
\hline 2006 & Snoopy vs. the Red Baron & Smart Bomb Interactive & $\begin{array}{l}\text { Namco Bandai } \\
\text { Games }\end{array}$ & $\begin{array}{l}\text { Simulateur de vol } \\
\text { arcade }\end{array}$ \\
\hline 2006 & $\begin{array}{c}\text { Wings of Honour: Battles of the Red } \\
\text { Baron }\end{array}$ & CITY interactive & CITY interactive & Simulateur de vol \\
\hline 2007 & 1914 Sbells of Fury & $\begin{array}{l}\text { Rondomedia ; h2 } \mathrm{f} \\
\text { Informationssysteme }\end{array}$ & Strategy First & $\begin{array}{l}\text { Simulateur de sous- } \\
\text { marin }\end{array}$ \\
\hline 2007 & Aggression: Europe Under Fire & Lesta Studio (?) & Buka Limited & Grande stratégie \\
\hline 2007 & Covert Front ${ }^{13}$ & Pastel Games & - & Aventure \\
\hline 2007 & The Darkness & Starbreeze Studios & 2K Games & FPS \\
\hline 2007 & WW1 Fighters & First Class Simulations & $\begin{array}{l}\text { First Class } \\
\text { Simulations }\end{array}$ & Simulateur de vol \\
\hline 2008 & Ace of aces & ? & ? & Simulateur de vol \\
\hline
\end{tabular}

12. Également connu sous le nom de WWI: The Great War.

13. Ce jeu sur navigateur comprend deux volets séparés, All Quiet on Covert Front et Station on the Horizon. 


\begin{tabular}{|c|c|c|c|c|}
\hline 2008 & Dogfight: The Great War ${ }^{14}$ & Rock Solid Games & Rock Solid Arcade & Side scroller \\
\hline 2008 & Mission: WWI Dogfight ${ }^{15}$ & First Class Simulations & Abacus Software & Simulateur de vol \\
\hline 2008 & $\begin{array}{c}\text { Secret Missions: Mata Hari and the } \\
\text { Kaiser's Submarines }\end{array}$ & $\begin{array}{l}\text { Frogwares Game } \\
\text { Development Studio }\end{array}$ & $\begin{array}{l}\text { Frogwares Game } \\
\text { Development Studio }\end{array}$ & Aventure \\
\hline 2008 & Warfare 1917 & ConArtist & Armor Games & Castle attack \\
\hline 2008 & World War One: La Grande Guerre ${ }^{16}$ & AGEod & $\begin{array}{c}\text { Ascaron } \\
\text { Entertainment }\end{array}$ & Grande tactique \\
\hline 2009 & $\begin{array}{c}\text { Boğaz Harbi: Canakkale Savasst } \\
\text { Destan }\end{array}$ & Kodgraf Oyun Stüdyosu & $\begin{array}{l}\text { Kodgraf Oyun } \\
\text { Stüdyosu }\end{array}$ & FPS \\
\hline 2009 & Darkest of Days & 8monkey Labs & Phantom EFX & FPS \\
\hline 2009 & Mata Hari & Cranberry Production & Viva Media & Aventure \\
\hline 2009 & NecroVisioN & The Farm 51 & $\begin{array}{l}\text { 1C Company ; } \\
505 \text { Games ; } \\
\text { Aspyr Media }\end{array}$ & FPS horreur \\
\hline 2009 & $\begin{array}{c}\text { Rise of Flight: The First Great Air } \\
\text { War }\end{array}$ & Neoqb ; 777 Studios & $\begin{array}{c}777 \text { Studios ; } \\
\text { Aerosoft ; ND } \\
\text { Games }\end{array}$ & Simulateur de vol \\
\hline 2009 & Supremacy 1914 & Bytro Labs & Bytro Labs & $\begin{array}{l}\text { Grande stratégie en } \\
\text { temps réel }\end{array}$ \\
\hline 2009 & Trenches & Thunder Game Works & $\begin{array}{l}\text { Thunder Game } \\
\text { Works }\end{array}$ & Castle attack \\
\hline 2009 & World of Tanks & Wargaming Minsk & Wargaming.net & Simulation de chars \\
\hline 2010 & NecroVisioN: Lost Company & The Farm 51 & 1C Company & FPS horreur \\
\hline 2010 & Snoopy Flying Ace & Smart Bomb Interactive & $\begin{array}{l}\text { Microsoft Game } \\
\text { Studios }\end{array}$ & $\begin{array}{l}\text { Simulateur de vol } \\
\text { arcade }\end{array}$ \\
\hline 2010 & Toy Soldiers & Signal Studios & $\begin{array}{l}\text { Microsoft Game } \\
\text { Studios }\end{array}$ & Tower defense \\
\hline 2010 & Victoria II & $\begin{array}{l}\text { Paradox Development } \\
\text { Studio }\end{array}$ & $\begin{array}{l}\text { Paradox Interactive } \\
\text { 1C/Snowball Studios }\end{array}$ & Grande stratégie \\
\hline 2011 & 1916 - Der Unbekannte Krieg & $\begin{array}{l}\text { Kriegsgraben und } \\
\text { Stormvogel }\end{array}$ & - & FPS horreur \\
\hline 2011 & Air Conflicts: Secret Wars & Games Farm & bitComposer & Simulateur de vol \\
\hline 2011 & Darkest Hour: A Hearts of Iron Game & Darkest Hour Team & Paradox Interactive & Grande stratégie \\
\hline 2011 & Strategic Command Classic: WWI & $\begin{array}{l}\text { Fury Software ; Matrix } \\
\text { Games }\end{array}$ & Matrix Games & Grande stratégie \\
\hline
\end{tabular}

14. Une suite du nom de Dogfight 2: The Great War est parue la même année.

15. Il ne s'agit pas d'un jeu à part entière mais d'un add-on pour Microsoft Flight Simulator X et 2004.

16. Une édition Gold améliorée est parue l'année suivante, puis une Centennial Edition augmentée en 2014, développée par Digital Froggies et éditée par Plug In Digital. 


\begin{tabular}{|c|c|c|c|c|}
\hline 2011 & The Snowfield & $\begin{array}{c}\text { Singapore-MIT Gambit } \\
\text { Game Lab }\end{array}$ & $\begin{array}{l}\text { Singapore-MIT } \\
\text { Gambit Game Lab }\end{array}$ & Action \\
\hline 2011 & Trenches 2 & Thunder Game Works & EA Games & Castle attack \\
\hline 2012 & Call of Ctbulbu: The Wasted Land & Red Wasp Design & Red Wasp Design & $\begin{array}{l}\text { RPG horreur / } \\
\text { tactique }\end{array}$ \\
\hline 2012 & Commander: The Great War & $\begin{array}{c}\text { The Lordz Games } \\
\text { Studio ; Slitherine ; } \\
\text { Matrix Games }\end{array}$ & $\begin{array}{l}\text { Slitherine; Matrix } \\
\text { Games }\end{array}$ & Grande stratégie \\
\hline 2013 & Sid Meier's Ace Patrol & Firaxis Games & 2K Games & Stratégie \\
\hline$(2014)^{17}$ & Fritz. & Truceful Entertainment & $\begin{array}{c}\text { Truceful } \\
\text { Entertainment }\end{array}$ & Aventure \\
\hline 2014 & Our World War: Interactive Episode & Mi & $\mathrm{BBC}$ & Aventure \\
\hline 2014 & $\begin{array}{c}\text { Soldats inconnus : Mémoires de la } \\
\text { Grande Guerre }\end{array}$ & Ubisoft Montpellier & Ubisoft & Aventure \\
\hline 2014 & Super Trench Attack! & Retro Army & Retro Army & Shooter \\
\hline 2014 & To End All Wars & AGEod & Slitherine & Grande stratégie \\
\hline 2014 & Wings Over Flanders Fields & OBD Software & OBD Software & Simulateur de vol \\
\hline 2015 & Assassin's Creed Syndicate & Ubisoft Quebec & Ubisoft & Action-aventure \\
\hline 2015 & Battle of Empires : 1914-1918 & Great War Team & Best Way Soft & RTS \\
\hline 2015 & Canvas Knights & Ilan Papini ; John Marco & $\begin{array}{l}\text { Ilan Papini ; John } \\
\text { Marco }\end{array}$ & Simulateur de vol \\
\hline 2015 & Don'Yoku & Dark-Spot Studio & Dark-Spot Studio & Shoot'em up \\
\hline 2015 & Iya Muromets & 1C Games Studios & 1C Games Studios (?) & Simulateur de vol \\
\hline 2015 & Making History: The Great War & $\begin{array}{c}\text { Muzzy Lane Software ; } \\
\text { Factus Games }\end{array}$ & Factus Games & Grande stratégie \\
\hline 2015 & Revolution Under Siege & Sep Reds ; AGEod & Slitherine & Grande stratégie \\
\hline 2015 & Spirit of War & G-OLD & Plug In Digital & Wargame \\
\hline 2015 & To Burn in Memory & Orihaus & Orihaus & Exploration \\
\hline 2015 & Toy Soldiers: War Chest & Signal Studios & Ubisoft & Tower defense \\
\hline 2015 & Verdun & $\begin{array}{c}\text { M2H ; } \\
\text { Blackmill Games }\end{array}$ & $\begin{array}{c}\text { M2H ; } \\
\text { Blackmill Games }\end{array}$ & FPS \\
\hline 2015 & World of Warships & $\begin{array}{l}\text { Wargaming.net ; Lesta } \\
\text { Studio }\end{array}$ & $\begin{array}{l}\text { Wargaming.net } \\
\text { Lesta Studio }\end{array}$ & Simulation maritime \\
\hline
\end{tabular}

17. Il s'agit d'un projet de jeu lancé en 2014 et dépendant d'une campagne de financement participatif. Celle-ci ayant échoué, le projet a été abandonné, seules quelques captures d'écran étant connues. Nous l'incluons cependant dans cette liste en raison de l'approche très particulière de la Grande Guerre qui transparaît à travers celles-ci. 


\begin{tabular}{|c|c|c|c|c|}
\hline 2016 & 1917 - The Alien Invasion DX & OtakuMaker.com & Andrade Games & Shoot 'em up \\
\hline 2016 & Aces High III & HiTech Creations & HiTech Creations & Simulateur de vol \\
\hline 2016 & Battlefield 1 & EA DICE & Electronic Arts & FPS \\
\hline $2016^{18}$ & Command Of War & Red Cube Games & Red Cube Games & Castle attack \\
\hline 2016 & Flying Baron 1916 & magnussoft & magnussoft & Simulateur de vol \\
\hline 2016 & Steam Squad & Bretwalda Games & Bretwalda Games & Stratégie \\
\hline 2016 & Tales from the Void & PortaPlay & Black Dog & Aventure \\
\hline 2016 & U-Boats & Astra Game Studio & Astra Game Studio & $\begin{array}{l}\text { Simulation de sous- } \\
\text { marin }\end{array}$ \\
\hline 2017 & Great War 1914 & Musrka & Blue Whale Games & Shooter \\
\hline 2017 & Supreme Ruler: The Great War & BattleGoat Studios & BattleGoat Studios & Grande stratégie \\
\hline 2017 & Tannenberg & $\begin{array}{c}\text { M2H ; } \\
\text { Blackmill Games }\end{array}$ & $\begin{array}{c}\text { M2H ; } \\
\text { Blackmill Games }\end{array}$ & FPS \\
\hline 2017 & The Operational Art of War IV & TrickeySoft LLC & Slitherine & Wargame \\
\hline 2018 & $\begin{array}{c}\text { Because We're Here } \sim \text { Mohnblume und } \\
\text { Blauerose }\end{array}$ & Studio Elfriede & Studio Elfriede & Visual novel \\
\hline 2018 & On The Western Front & Aggroblakh & Aggroblakh & Stratégie \\
\hline 2018 & Revolt 1917 & juhbee & juhbee & FPS \\
\hline 2018 & Soldiers Lost Forever (1914-1918) & Jake Olson & Impartial Studios & Castle attack \\
\hline 2018 & Temporality & James Earl Cox III & James Earl Cox III & Exploration \\
\hline 2018 & $\begin{array}{c}\text { Wars Across The World: Russian } \\
\text { Battles }\end{array}$ & Strategiae & Plug In Digital & Grande stratégie \\
\hline 2019 ?19 & The Trench 1916 & Gallica Game Studio & Gallica Game Studio & FPS \\
\hline 2019 & Within Whispers: The Fall & Thorium Studios & Thorium Studios & Aventure \\
\hline$? 20$ & Ad Infinitum & StrixLab & StrixLab & Aventure horreur \\
\hline$? 21$ & Cry of War & ShanghaiWindy & ShanghailWindy & Simulation de chars \\
\hline
\end{tabular}

18. Il s'agit d'un projet de jeu lancé en 2016 et dépendant d'une campagne de financement participatif. Celle-ci ayant échoué, le projet semble avoir été abandonné, mais une version de démonstration avait été publiée alors.

19. En développement par une très petite équipe depuis presque une décennie, la date de sortie de ce jeu a été repoussée de nombreuses fois, la dernière annonce à ce jour datant de mai 2018 et prévoyant une première version pour 2019.

20. Ce jeu est en développement depuis quelques années et aucune date de sortie ne semble avoir été annoncée.

21. Ce jeu est en développement actif, une version alpha étant déjà disponible. 


\section{TABLEAU 2}

Liste des armements individuels représentés de façon identifiable dans les jeux du corpus. Dans l'ensemble des tableaux suivants, les dates données sont celles de mise en service. Les armements placés entre crochets correspondent à des projets ou prototypes n'ayant pas été mis en production, la date étant alors celle du projet. Les armes d'infanterie et d'artillerie n'ont été reportées que lorsqu'il est possible d'identifier une arme assez précise : en effet tous les jeux de guerre proposent des fusils, mitrailleuses ou canons, mais souvent génériques. De plus, lorsqu'un modèle est précisé, ne sont souvent donnés que le type et le diamètre, non le modèle exact qu'il est donc nécessaire de déduire par recoupement, avec les risques d'erreur induits. Concernant les armes individuelles, nous distinguons selon l'usage de l'époque entre fusilmitrailleur, fusil automatique (que l'on peut assimiler au fusil d'assaut) et pistolet-mitrailleur. Sans entrer dans les détails techniques, le premier type est une arme lourde utilisée en position fixe, habituellement appelée " mitrailleuse » et typique de la Grande Guerre ; au contraire les deux suivants, le dernier correspondant à la " mitraillette ", sont des armes portatives plus légères utilisées en mouvement, utilisées seulement très ponctuellement voire à titre expérimental durant le conflit. De même, le "revolver " désigne une arme de poing à chargement automatique par un barillet tournant, tandis que le pistolet comporte un chargeur "linéaire " à ressort. Nous incluons les mortiers et les canons d'infanterie dans les armes d'infanterie puisque, si ceux-ci pouvaient être aussi imposants que des pièces d'artillerie "classiques ", ils étaient mis en œuvre directement depuis les tranchées. Enfin, nous n’indiquons les armes blanches que lorsque celles-ci comportent une référence réglementaire claire (nous excluons donc les armes de fortune ou encore le sabre traditionnel russe appelé chachka, présent dans Tannenberg (2017). 


\begin{tabular}{|c|c|c|c|c|c|c|c|c|c|c|c|c|c|}
\hline 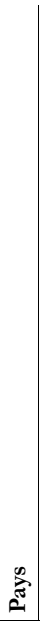 & Type & Désignation (année) & 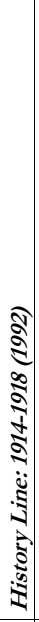 & 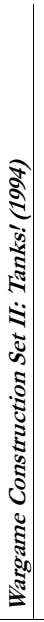 & 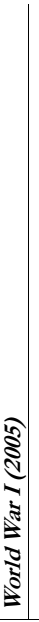 & 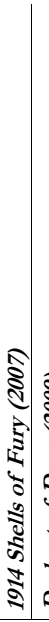 & 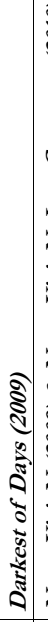 & 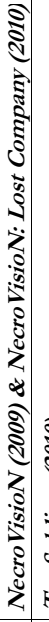 & 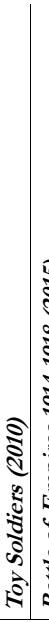 & 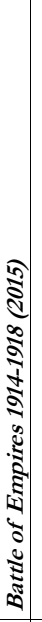 & 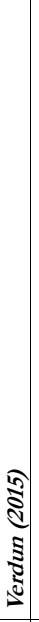 & 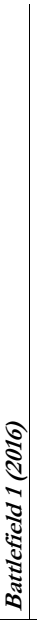 & 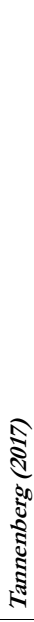 \\
\hline \multirow{18}{*}{ 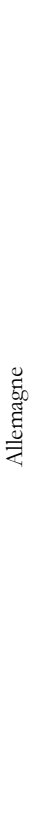 } & \multirow{2}{*}{ Arme blanche } & Infanterie-Offizierssäbel M1861 (1861) & & & & & & & & & & & $\mathrm{x}$ \\
\hline & & M1887 Feldspaten $(1887)^{1}$ & & & & & & & & & $\mathrm{x}$ & $\mathrm{x}$ & $\mathrm{x}$ \\
\hline & $\begin{array}{c}\text { Canon } \\
\text { automatique }\end{array}$ & Becker Type M2 20mm Cannon (1913)² & & & & & & & & & & $\mathrm{x}$ & \\
\hline & \multirow{6}{*}{ Fusil et assimilés } & Karabiner 88 (1888) & & & & & & & & & & & $\mathrm{x}$ \\
\hline & & Gewehr $98(1895)^{3}$ & & & & & $\mathrm{x}$ & $\mathrm{x}$ & & & $\mathrm{x}$ & $\mathrm{x}$ & $\mathrm{x}$ \\
\hline & & Infanterie Repetier-Gewehr M.95 (1895) ${ }^{4}$ & & & & & & & & & & & $\mathrm{x}$ \\
\hline & & Repetier-Stutzen M1895 (1895) & & & & & & & & & & & $\mathrm{x}$ \\
\hline & & Gewehr 88/05 (1905) & & & & & & & & & & & $\mathrm{x}$ \\
\hline & & Karabiner Model 1898 AZ (1908) & & & & & & & & & $\mathrm{x}$ & & $\mathrm{x}$ \\
\hline & Fusil antichar & $\begin{array}{c}3.7 \text { cm Tankabwehrkanone } 1918 \text { (1918) } \\
\text { Tankgewehr M1918 (1918) }\end{array}$ & $\mathrm{x}$ & $\mathrm{x}$ & & & & & & & & $\mathrm{x}$ & \\
\hline & \multirow{3}{*}{ Fusil automatique } & [Luger Selbstlader Model 06 (1906)] & & & & & & & & & & $\mathrm{x}$ & \\
\hline & & Mondragón Modelo $1908(1908)^{5}$ & & & & & & & & & & $\mathrm{x}$ & \\
\hline & & [Selbstlader-Karabiner M1916 (1916)] & & & & & & & & & & $\mathrm{x}$ & \\
\hline & \multirow{3}{*}{ Fusil-mitrailleur } & Parabellum MG 14 (1914) & & & & & & & & & & $\mathrm{x}$ & \\
\hline & & Bergmann MG 15 (1916) & & & & & & & & & & $\mathrm{x}$ & \\
\hline & & Maschinengewehr 08/15 (1916) ${ }^{6}$ & & & $\mathrm{x}$ & $\mathrm{x}$ & & $\mathrm{x}$ & $\mathrm{x} \mid \mathrm{y} \quad \ln ^{2}$ & $\mathrm{x}$ & $\mathrm{x}$ & $\mathrm{x}$ & \\
\hline & \multirow[b]{2}{*}{ Grenade } & Discushandgranate 1915 (1915) & & & & & & & & & & $\mathrm{x}$ & \\
\hline & & $\begin{array}{l}\text { Stielhandgranate Model } 1915 \text { (1915) } \\
\text { Model } 17 \text { Eierhandgranate (1916) }\end{array}$ & & & & & & $\mathrm{x}$ & & & $\mathrm{x}$ & $\mathrm{x}$ & $\mathrm{x}$ \\
\hline
\end{tabular}

1. Bien que ne s'agissant pas à proprement parler d'une arme, cette pelle de tranchée allemande est suffisamment iconique des combats de la Grande Guerre pour être considérée comme telle.

2. Nous n'avons pu identifier la dénomination allemande exacte de ce canon automatique.

3. Habituellement connu sous le nom de Mauser, qui désigne en réalité son manufacturier.

4. Habituellement connu sous le nom de fusil Mannlicher.

5. Fusil d'assaut de fabrication mexicaine.

6. Habituellement connu sous le nom de mitrailleuse Maxim. 


\begin{tabular}{|c|c|c|c|c|c|c|c|c|c|c|}
\hline & Stielhandgranate Model 1917 (1917) & & & & & & & & $\mathrm{x}$ & $\mathrm{x}$ \\
\hline & Stielhandgranate Model 1917 Geballte ladung $(1917)^{7}$ & & & & $\mathrm{x}$ & & & & $\mathrm{x}$ & $\mathrm{x}$ \\
\hline Lance-flammes & Wechselapparat (1917) & & & & & & & & $\mathrm{x}$ & \\
\hline & $25 \mathrm{~cm}$ schwerer Minenwerfer (1910) & & $\mathrm{x}$ & & & & & & & \\
\hline & $17 \mathrm{~cm}$ mittlerer Minenwerfer (1913) & & $\mathrm{x}$ & & & & & & & \\
\hline Mortier & $7.58 \mathrm{~cm}$ Minenwerfer $(1914)^{8}$ & $\mathrm{x}$ & & & & & $\mathrm{x}$ & & & \\
\hline & $9.15 \mathrm{~cm}$ leichtes Minenwerfer System Lanz (1915) & & $\mathrm{x}$ & & & & & & & \\
\hline & Granatenwerfer 16 (1916) & & & & & $\mathrm{x}$ & $\mathrm{x}$ & & & \\
\hline & Borchardt C-93 (1895) & & & & & & & & $\mathrm{x}$ & \\
\hline & Mauser C96 (1896) & & & & & & & & $\mathrm{x}$ & $\mathrm{x}$ \\
\hline ristoret & Lange Pistole $08(1913)^{9}$ & & & $\mathrm{x}$ & $\mathrm{x}$ & & & $\mathrm{x}$ & $\mathrm{x}$ & $\mathrm{x}$ \\
\hline & Mauser Model 1914 (1914) & & & & & & & & $\mathrm{x}$ & \\
\hline & Bergmann Maschinenpistole 18 (1918) & & & & $\mathrm{x}$ & & & $\mathrm{x}$ & $\mathrm{x}$ & \\
\hline 1. & [08/18 Maxim SMG (1918 ?)] ${ }^{10}$ & & & & & & & & $\mathrm{x}$ & \\
\hline Revolver & Reichsrevolver Modell 1883 (1883) & & & & & & & & & $\mathrm{x}$ \\
\hline Canon d'infanterie & 37mm Infanteriegeschütz M.15 (1915) ${ }^{11}$ & & & & & & $\mathrm{x}$ & & & \\
\hline & Mannlicher M1888-90 (1890) & & & & & & & & & $\mathrm{x}$ \\
\hline Fusil et assimilé & Mannlicher M1893 (1893) & & & & & & & & & $\mathrm{x}$ \\
\hline & Mannlicher Model 1893 Carbine (1893) & & & & & & & & & $\mathrm{x}$ \\
\hline$\Gamma_{\text {- il }}$ & Madsen machine gun $(1902)^{12}$ & & & & & & & & & \\
\hline 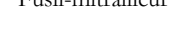 & Schwarzlose Maschinengewehr Modell 07 (1907) & & $\mathrm{x}$ & & & & $\mathrm{x}$ & & & \\
\hline Grenade & Grenade Rohr $(1915)^{13}$ & & & & & & & & & $\mathrm{x}$ \\
\hline & 9 cm Minenwerfer M 14 (1914) & & $\mathrm{x}$ & & & & & & & \\
\hline Mortiers & $26 \mathrm{~cm}$ Minenwerfer M $17(1917)^{14}$ & & $\mathrm{x}$ & & & & & & & \\
\hline & 7.5cm Minenwerfer M.17 (1917) & & & & & & $\mathrm{x}$ & & & \\
\hline & Frommer Stop (1912) & & & & & & & & $\mathrm{x}$ & \\
\hline Pistolet & Steyr M12 P16 (1912) & & & & & & & & $\mathrm{x}$ & $\mathrm{x}$ \\
\hline & 2mm Kolibri (1914) ${ }^{15}$ & & & & & & & & $\mathrm{x}$ & \\
\hline Pistolet-mitrailleur & [Standschütze Hellriegel M1915 (1915)] & & & & & & & & $\mathrm{x}$ & \\
\hline
\end{tabular}

7. Il s'agit d'une grenade Stielhandgranate Model 1917 avec plusieurs têtes explosives rassemblées. Le jeu Tannenberg (2017) en distingue des versions à six et neuf têtes.

8. Le jeu Wargame Construction Set II: Tanks! (1994) comporte pour l'armée allemande de la Première Guerre mondiale un "mortier de $90 \mathrm{~mm}$ ", pièce que nous ne sommes pas parvenu à identifier. Nous l'assimilons, faute de mieux, au 7,58 cm Minenwerfer.

9. Habituellement connu sous le nom de Luger.

10. Cette arme n'est attestée que par quelques exemplaires survivants, mais ni son histoire ni son éventuelle désignation ne sont connues.

11. Dans le jeu Toy Soldiers (2010), un canon pouvant correspondre à celui-ci est jouable dans l'armée allemande.

12. Fusil-mitrailleur léger de fabrication danoise. Dans le jeu Battlefield 1 (2016), celui-ci est joué dans les armées autrichienne et ottomane, mais il a en réalité été fourni à la plupart des armées en conflit dans les deux camps.

13. Nous n'avons pu identifier la dénomination officielle de cette grenade, nommée d'après son inventeur. Le jeu Tannenberg (2017) distingue des versions offensive et offensive de celle-ci.

14. Le jeu World War I (2005) fait figurer dans les armées des empires centraux un " mortier lourd de $280 \mathrm{~mm}$ ". D'après son graphisme, il semble s'approcher du $26 \mathrm{~cm}$ Minenwerfer M 17, bien que celui-ci soit de $260 \mathrm{~mm}$.

15. Il s'agit d'une arme d'autodéfense à destination du public civil, qui n’a probablement jamais été employée sur un champ de bataille. 


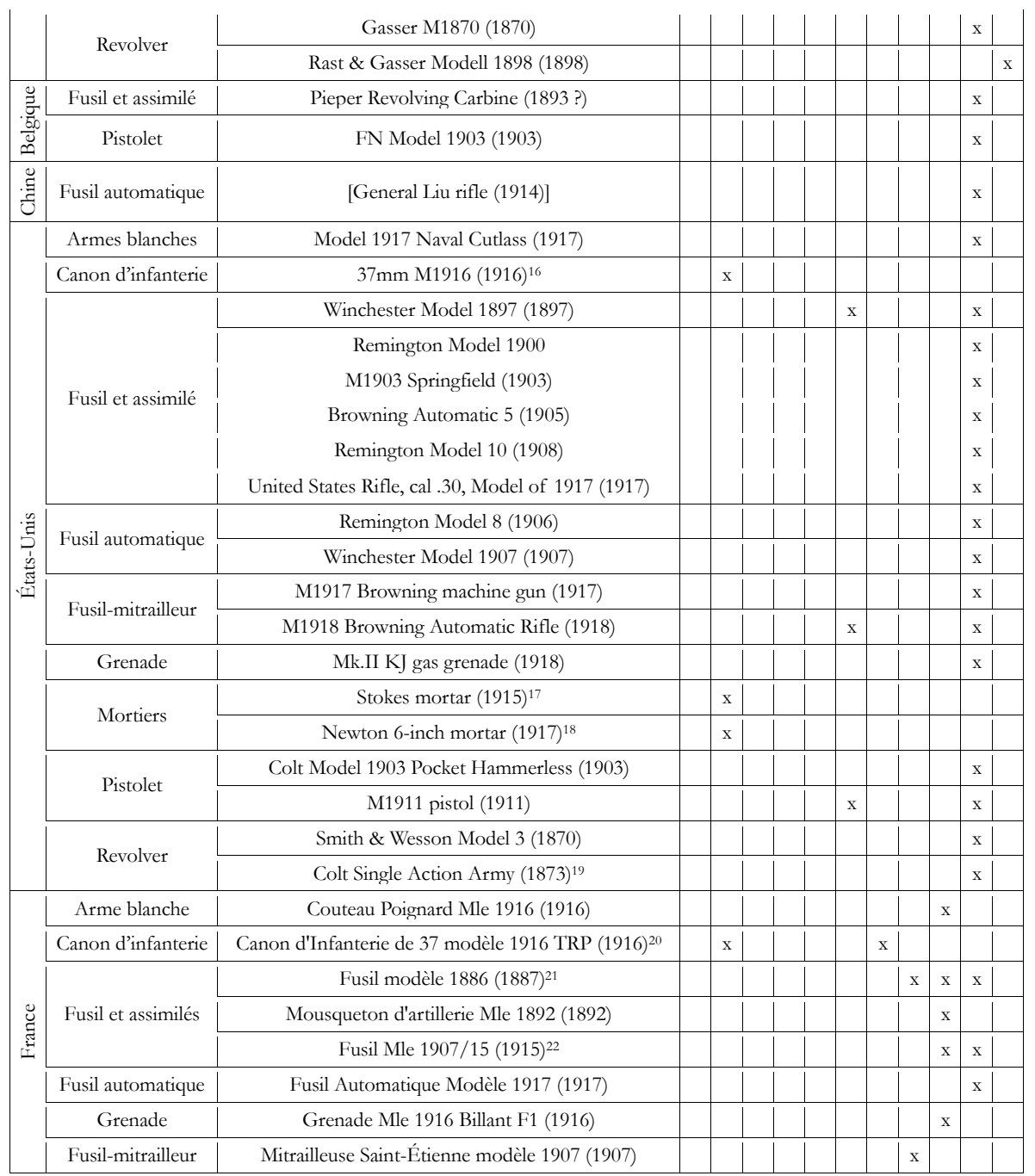

16. Désignation américaine du Canon d'Infanterie de 37 modèle 1916 TRP fourni par la France.

17. Le jeu Wargame Construction Set II: Tanks! (1994) comporte pour l'armée américaine de la Première Guerre mondiale un "mortier de 3 pouces ", probablement un Stokes mortar de fourniture britannique.

18. Le jeu Wargame Construction Set II: Tanks! (1994) comporte pour l'armée américaine de la Première Guerre mondiale un "mortier de 6 pouces ", probablement un Newton 6-inch mortar britannique, copié par l'armée américaine à la fin de la guerre, mais dont la réalité de l'usage sur le terrain n'est pas assurée.

19. Habituellement connu sous le nom de Colt Peacemaker.

20. Dans le jeu Toy Soldiers (2010), cette arme peut être journée dans l'armée britannique, apparemment sans raison historique.

21. Habituellement désigné comme fusil Lebel.

22. Habituellement désigné comme fusil Berthier. 


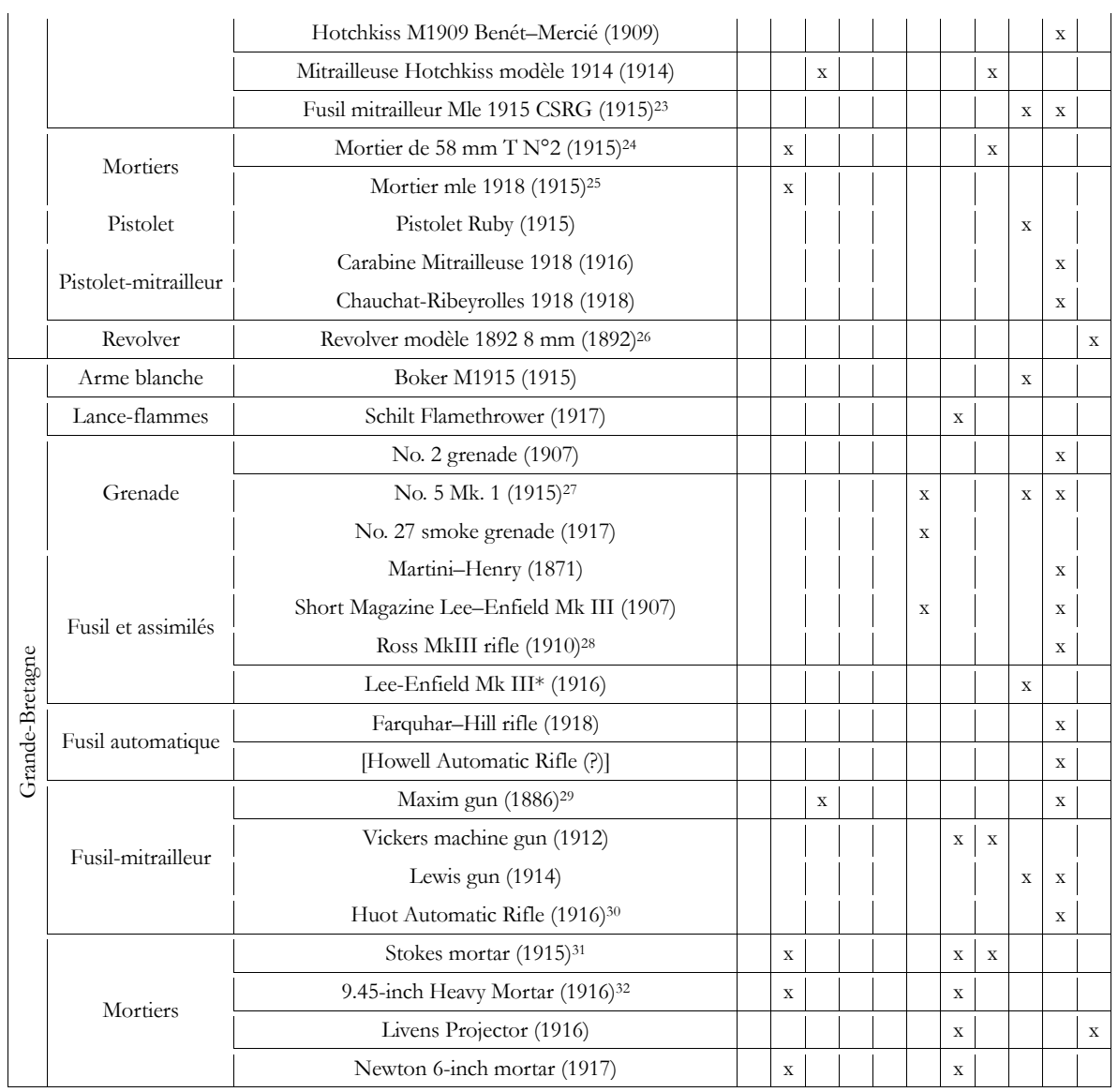

23. Habituellement désigné comme fusil mitrailleur Chauchat.

24. Ce mortier est généralement connu en France par son surnom de "crapouillot ». Dans le jeu World War I (2005), ce mortier est joué dans l'armée russe, a priori sans raison historique.

25. Également connu sous le nom de " mortier de 3 pouces ", il s'agit du Stokes mortar britannique en service dans l'armée française.

26. Dans le jeu Tannenberg (2017), ce revolver est jouable dans l'armée russe, apparemment sans fondement historique.

27. Habituellement désignée comme Mills Bomb.

28. Cette arme était de fabrication canadienne.

29. Le jeu World War I (2005) fait figurer dans l'armée britannique une " mitrailleuse de 7,62 mm ». D'après son graphisme, il pourrait s'agir du Maxim Gun, bien que celui-ci tire des munitions .303 British, soit $7,70 \mathrm{~mm}$.

30. Arme canadienne, employée par le corps expéditionnaire canadien.

31. Le jeu Wargame Construction Set II: Tanks! (1994) comporte pour l'armée britannique de la Première Guerre mondiale un " mortier de 3 pouces ", et le jeu World War I (2005) un " mortier de 75 mm ", pièces qui correspondent probablement au Stokes mortar, lequel étant techniquement bien nominalement un mortier de 3 pouces (soit $76,2 \mathrm{~mm}$ ), quoique très légèrement plus large concrètement.

32. Le jeu Wargame Construction Set II: Tanks! (1994) comporte pour l'armée britannique de la Première Guerre mondiale un "mortier de 9,5 pouces ", pièce qui correspond probablement à une simplification de la nomenclature du 9.45-inch Heavy Mortar. 


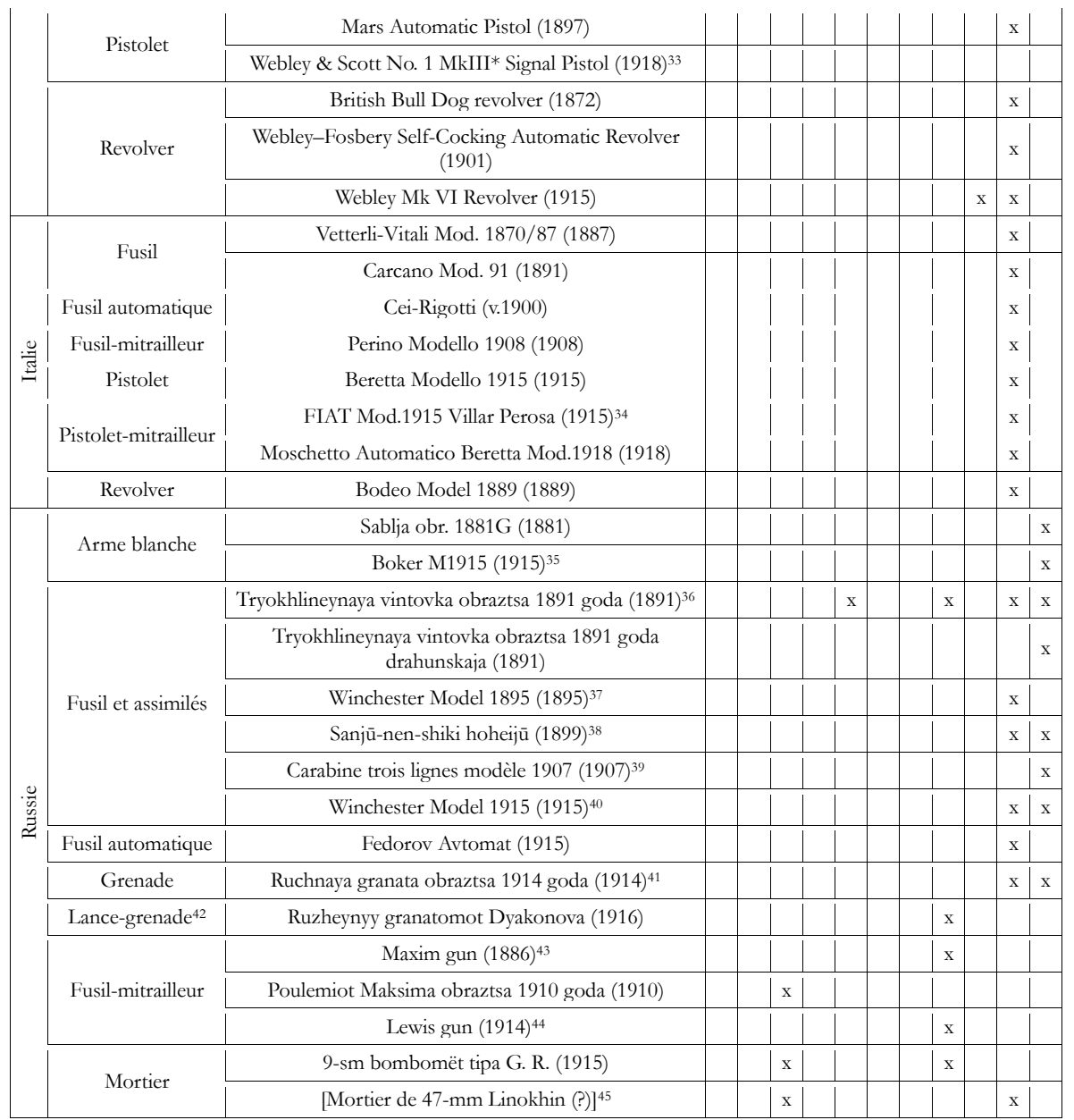

33. Il s'agit d'un pistolet de signalement, tirant des fusées éclairantes ou de communication.

34. Bien que techniquement un pistolet-mitrailleur, cette arme a été conçue pour un emploi fixe, plus proche de celui des fusils-mitrailleurs.

35. Couteau de tranchée de fourniture britannique.

36. Généralement connu sous le nom de Mosin-Nagant.

37. Arme fabriquée aux États-Unis pour l'armée russe.

38. Habituellement connu sous le nom de Arisaka, il s'agit d'un fusil japonais, fourni à l'armée russe via la Grande-Bretagne.

39. Nous n'avons pu identifier avec certitude le nom russe de cette carabine Mosin-Nagant.

40. Carabine fournie par les États-Unis.

41. Le jeu Tannenberg (2017) distingue des versions offensive et offensive de cette grenade.

42. Il s'agit d'un lance-grenade à adapter sur le canon d'un fusil.

43. Arme de fourniture britannique.

44. Arme de conception américaine et de construction britannique, fournie par ce dernier pays.

45. Le jeu World War I (2005) mentionne une telle arme pour l'armée russe. Nous ne sommes pas parvenu à l'identifier, mais la présence d'un nom particulier laisserait supposer qu'il s'agit au moins d'un projet ayant bien existé (à moins que ce nom fasse référence à un scénario du jeu et que l'arme soit totalement fictive). Nous l'incluons donc dans le tableau sous réserve. 


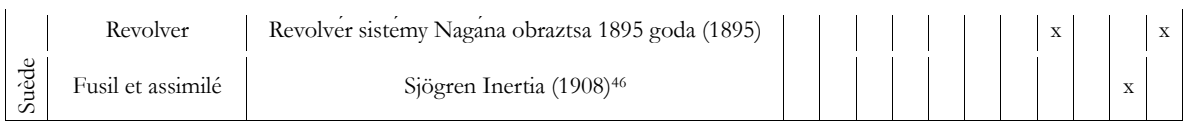

46. Ce fusil à pompe suédois a été utilisé très ponctuellement par la Triple Alliance aussi bien que la Triple Entente. 


\section{TABLEAU 3}

Liste des pièces d'artillerie représentées de façon identifiable dans les jeux du corpus. La limite entre l'artillerie de campagne et l'artillerie lourde n'est pas clairement fixée. Nous la plaçons arbitrairement à $120 \mathrm{~mm}$.

\begin{tabular}{|c|c|c|c|c|c|c|c|c|c|}
\hline$\overbrace{i}^{\infty}$ & Type & Désignation (année) & 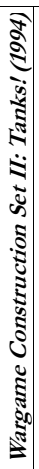 & 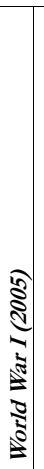 & 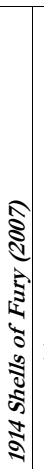 & 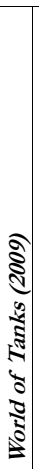 & 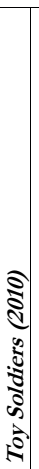 & 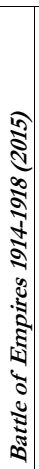 & 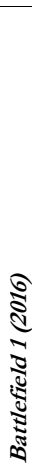 \\
\hline \multirow{21}{*}{ 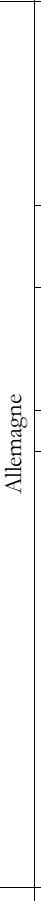 } & \multirow{5}{*}{ Artillerie de campagne } & $8.8 \mathrm{~cm} \mathrm{SK} \mathrm{L/30} \mathrm{(1892)^{1 }}$ & & & $\mathrm{x}$ & & & & \\
\hline & & $7.7 \mathrm{~cm}$ Feldkanone 96 neuer Art (1905) & & & & & $\mathrm{x}$ & & $\mathrm{x}$ \\
\hline & & $15 \mathrm{~cm} \mathrm{SK} \mathrm{L/45} \mathrm{(1908)}$ & & & & & & & $\mathrm{x}$ \\
\hline & & Skoda 7.5 cm Gebirgskanone M. 15 (1915) & & $\mathrm{x}$ & & & & & \\
\hline & & $7.7 \mathrm{~cm}$ Feldkanone 16 (1916) & $\mathrm{x}$ & $\mathrm{x}$ & & & & $\mathrm{x}$ & \\
\hline & \multirow{2}{*}{ Artillerie lourde } & $15 \mathrm{~cm}$ Kanone 16 (1917) & $\mathrm{x}$ & & & & & & \\
\hline & & $21 \mathrm{~cm}$ Schnelladekanone Länge 45 (1909) & $\mathrm{x}$ & & & & & & \\
\hline & \multirow{3}{*}{ Artillerie anti-aérienne } & 3,7-cm-MaschinenFlak M 14 (1914) & & & & & $\mathrm{x}$ & & \\
\hline & & 8.8cm Flak 17 (1917) & & & & & $\mathrm{x}$ & & \\
\hline & & Revolverkanone Lichtspucker $37 \mathrm{~mm}(?)^{2}$ & & & & & $\mathrm{x}$ & & \\
\hline & \multirow[t]{11}{*}{ Artillerie antl-aerienne autopropulsee } & 7.7 cm Leichte Kraftwagengeschütze M1914 (1914) & & $\mathrm{x}$ & & & & & \\
\hline & & $15 \mathrm{~cm}$ schwere Feldhaubitze $1902(1903)^{3}$ & & $\mathrm{x}$ & & & & & \\
\hline & & Krupp 7.5 cm Model $1903(1904)^{4}$ & $\mathrm{x}$ & & & & & & \\
\hline & & $10.5 \mathrm{~cm}$ Feldhaubitze 98/09 (1909) & & & & & & $\mathrm{x}$ & \\
\hline & & 21 cm Mörser 10 (1911) & & $\mathrm{x}$ & & & & & \\
\hline & & Škoda 30.5 cm Mörser M.11 (1911) & & $\mathrm{x}$ & & & & & \\
\hline & & $15 \mathrm{~cm}$ schwere Feldhaubitze 13 (1913) & & & & & & $\mathrm{x}$ & \\
\hline & & $\begin{array}{c}\text { 42-cm Kurze Marine-Kanone } 14 \text { (M-Gerät) « Dicke } \\
\text { Bertha » (1914) }\end{array}$ & & $\mathrm{x}$ & & & $\mathrm{x}$ & & \\
\hline & & $38 \mathrm{~cm} \mathrm{SK} \mathrm{L/45} \mathrm{«Max} \mathrm{»} \mathrm{(1915)}$ & & $\mathrm{x}$ & & & & & \\
\hline & & $10.5 \mathrm{~cm}$ leichte Feldhaubitze 16 (1916) & $\mathrm{x}$ & $\mathrm{x}$ & & & & $\mathrm{x}$ & \\
\hline & & 21 cm Mörser 16 (1916) & & $\mathrm{x}$ & & & $\mathrm{x}$ & & \\
\hline & Artillerie de campagne & $8 \mathrm{~cm}$ Feldkanone M 05 (1907) & & $\mathrm{x}$ & & & & $\mathrm{x}$ & \\
\hline
\end{tabular}

1. Il s'agit d'une pièce d'artillerie navale installée sur les navires et sous-marins.

2. Cette pièce a bien existé mais est mal documentée. Ayant un fonctionnement de revolver, elle disposait de cinq canons tournants et correspondait à ce que les pilotes alliés avaient surnommé les " flaming onions ». Le surnom allemand était "Lichtspucker " mais nous ne sommes pas parvenu à identifier la désignation officielle. 3. Le jeu World War I (2005) fait figurer pour les empires centraux un "obusier de $150 \mathrm{~mm}$ " daté de 1904 . Nous supposons qu'il s'agit du $15 \mathrm{~cm}$ schwere Feldhaubitze 1902, bien que celui-ci soit entré en service l'année précédente.

4. Le jeu Wargame Construction Set II: Tanks! (1994) comporte pour l'armée allemande de la Première Guerre mondiale un "obusier de $76 \mathrm{~mm}$ ", pièce que nous ne sommes pas parvenu à identifier. Nous l'assimilons, faute de mieux, au Krupp 7,5 cm Model 1903. 


\begin{tabular}{|c|c|c|c|c|c|c|c|}
\hline \multirow{3}{*}{ 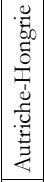 } & & Skoda 7.5 cm Gebirgskanone M. 15 (1915) & & & & & $\mathrm{x}$ \\
\hline & \multirow[b]{2}{*}{ Obusier } & $18 \mathrm{~cm}$ kurze Kanone M $80(1881)^{5}$ & & $\mathrm{x}$ & & & \\
\hline & & 10 cm Feldhaubitze M.14 (1914) & & & & & $\mathrm{x}$ \\
\hline \multirow{5}{*}{ 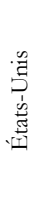 } & \multirow{4}{*}{ Artillerie de campagne } & Canon de 120 mm L modèle $1878(1878)^{6}$ & $\mathrm{x}$ & & & & \\
\hline & & Canon de 75 mm modèle $1897(1898)^{7}$ & $\mathrm{x}$ & & & & \\
\hline & & 4.7 inch Gun M1906 (1907) & $\mathrm{x}$ & & & & \\
\hline & & 75 mm Gun M1916 (1918) & & $\mathrm{x}$ & & & \\
\hline & Artillerie lourde & M1917A1 $(1917)^{9}$ & $\mathrm{x}$ & & & & \\
\hline \multirow{12}{*}{ 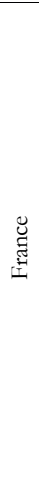 } & \multirow{7}{*}{ Artillerie de campagne } & Canon Lahitolle de 95 mm (1875) & $\mathrm{x}$ & & & & \\
\hline & & Canon de Bange de $80 \mathrm{~mm}$ (1877) & & $\mathrm{x}$ & & & \\
\hline & & Canon de 120 mm L modèle 1878 (1878) & $\mathrm{x}$ & & & & \\
\hline & & QF 4.7 inch Gun $(1887)^{10}$ & $\mathrm{x}$ & & & & \\
\hline & & Canon de 75 mm modèle 1897 (1898) & $\mathrm{x}$ & $\mathrm{x}$ & & & $\mathrm{x}$ \\
\hline & & Canon de $105 \mathrm{~mm}$ modèle 1913 Schneider $(1900)^{11}$ & & $\mathrm{x}$ & & & \\
\hline & & Canon de 37 mm TR Mle 16 (1916) & & & & & $\mathrm{x}$ \\
\hline & Artillerie lourde & Canon de 155 mm L modèle 1877 (1877) & $\mathrm{x}$ & & & & \\
\hline & Artillerie antiaérienne & Canon de 75 mm modèle $1897(1897)^{12}$ & & & & $\mathrm{x}$ & \\
\hline & Artillerie autopropulsée & Renault FT 75BS (1918) & & & $\mathrm{x}$ & & \\
\hline & \multirow{2}{*}{ Obusier } & Mortier de $280 \mathrm{~mm}$ Schneider $(1914)^{13}$ & & $\mathrm{x}$ & & & \\
\hline & & Obusier de $400 \mathrm{~mm}$ (1916) & & $\mathrm{x}$ & & & \\
\hline \multirow{5}{*}{ 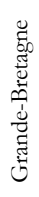 } & \multirow{5}{*}{ Artillerie de campagne } & QF 3-pounder Hotchkiss (1886) & & & & & $\mathrm{x}$ \\
\hline & & QF 4.7 inch Gun (1887) & $\mathrm{x}$ & & & & \\
\hline & & QF 2.95-inch Mountain Gun $(1897)^{14}$ & & $\mathrm{x}$ & & & \\
\hline & & Ordnance QF 15-pounder (1901) & $\mathrm{x}$ & & & & \\
\hline & & Ordnance QF 18-pounder (1904) & $\mathrm{x}$ & & & $\mathrm{x}$ & $\mathrm{x}$ \\
\hline
\end{tabular}

5. En principe un obusier sur roues, cette pièce semble avoir pu être démontée et placée sur une plate-forme fixe improvisée pour en faire un mortier.

6. Nous n'avons pas trouvé d'attestation d'un usage de cette pièce française dans l'armée américaine. Sa présence dans le manuel du jeu Wargame Construction Set II: Tanks! (1994) pourrait donc être une erreur, d'autant qu'il serait redondant avec le canon américain de 4,7 inch aux caractéristiques proches.

7. Il s'agit d'une pièce française fournie à l'armée américaine.

8. Cette pièce est arrivée trop tard en France pour être employée au combat. Le jeu World War I (2005) fait figurer pour l'Entente un canon de 75 mm antichar monté à l'arrière d'un camion. Le 75 mm Gun M1916 ayant bien été employé dans cette configuration, il pourrait s'agir de cette pièce (quoique dans la mauvaise armée et de manière anachronique).

9. Désignation américaine du Canon de 155 C modèle 1917 Schneider fourni par l'armée française, lequel était techniquement un obusier.

10. Ce canon britannique a été utilisé en France sur des bâtiments de marine, mais nous n’avons pas trouvé d'attestation d'un usage à terre. Sa présence dans le manuel du jeu Wargame Construction Set II: Tanks! (1994) pourrait donc être une erreur, d'autant qu'il serait redondant avec le canon français de $120 \mathrm{~mm}$ aux caractéristiques proches.

11. Malgré sa désignation (due à un achat par l'armée française en 1913 seulement), cette pièce a bien été conçue en 1900.

12. Pièce identique à celle employée comme artillerie de campagne, seuls les obus étaient antiaériens. Dans le jeu Toy Soldiers (2010), elle est jouable dans l'armée britannique, apparemment sans raison historique.

13. Malgré sa désignation, cette pièce était techniquement un obusier.

14. Le jeu World War I (2005) fait figurer un « canon de 82,5 mm » dans le matériel de l'Entente. D'après son apparence graphique, il pourrait s'agir du QF 2.95-inch Mountain Gun, bien que celui-ci soit une pièce de $75 \mathrm{~mm}$. 


\begin{tabular}{|c|c|c|c|c|c|c|c|c|c|}
\hline & & Ordnance QF 3-pounder Vickers (1905) ${ }^{15}$ & & $\mathrm{x}$ & & & & & \\
\hline & 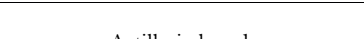 & Ordnance BL 60-pounder (1905) & $\mathrm{x}$ & & & & & & \\
\hline & Artillerie lourde & 5"/51 BL Mark VI (1911) $)^{16}$ & & $\mathrm{x}$ & & & & & \\
\hline & & QF 1 pounder (v.1890) ${ }^{17}$ & & & & & & & $\mathrm{x}$ \\
\hline & Artillerie anti-aerienne & QF 2-pounder (1915) ${ }^{18}$ & & & & & $\mathrm{x}$ & & \\
\hline & - t. & Pierce-Arrow Anti-Aircraft Armoured Lorry (1914) & & & & & & & $\mathrm{x}$ \\
\hline & Artuene ant-aeriente autopropusee & QF 13-pounder 6 cwt AA (1916) ${ }^{19}$ & & & & & $\mathrm{x}$ & & \\
\hline & & QF 4.5-inch howitzer (1908) & $\mathrm{x}$ & & & & & $\mathrm{x}$ & \\
\hline & & BL 9.2-inch howitzer (1915) & & & & & $\mathrm{x}$ & & $\mathrm{x}$ \\
\hline & Obusiers & BL 15-inch howitzer (1915) & & $\mathrm{x}$ & & & & & \\
\hline & & L 6-inch 26 cwt howitzer (1915) & $\mathrm{x}$ & & & & & & \\
\hline & & BL 8-inch howitzer Mk VI (1916) & & & & & $\mathrm{x}$ & & \\
\hline & & $\begin{array}{l}\text { 76-mm divizionnaya pushka obraztsa } 1902 \text { goda } \\
\text { (1903) }\end{array}$ & & $\mathrm{x}$ & & & & $\mathrm{x}$ & \\
\hline & Artillerie de campagne & 107-mm pushka obraztsa 1910 goda (1910) & & $\mathrm{x}$ & & & & & \\
\hline & & Transheynaya 37-mm pushka obr. 1915 goda (1915) & & & & & & $\mathrm{x}$ & \\
\hline$\infty$ & A trillorip lound & $254 \mathrm{~mm} 45$ caliber Pattern $1891(1897)^{20}$ & & $\mathrm{x}$ & & & & & \\
\hline & Artillerie lourde & 12"/52 pushka Obukhovskogo zavoda (1907) & & & & & & & $\mathrm{x}$ \\
\hline & Artillerie anti-aérienne & 3" zenitnaya pushka obr. 1914/1915 goda (1915) & & $\mathrm{x}$ & & & & & \\
\hline & OI & 122-mm gaubica obr. 1909 gg. $(1909)^{21}$ & & $\mathrm{x}$ & & & & $\mathrm{x}$ & \\
\hline & Obuster & $6 \mathrm{dm}$ polevaja gaubitsa sistemy Schneidera (1910) & & $\mathrm{x}$ & & & & & \\
\hline
\end{tabular}

15. Le jeu World War I (2005) fait figurer un « canon Vickers de $44 \mathrm{~mm}$ » dans le matériel de l'Entente. Nous supposons qu'il s'agit du Ordnance QF 3-pounder Vickers, bien qu'il s'agisse plutôt d'une pièce navale et que son diamètre soit de $47 \mathrm{~mm}$.

16. Le jeu World War I (2005) fait figurer un " canon de $127 \mathrm{~mm}$ » dans le matériel de l'Entente. La seule pièce semblant correspondre historiquement serait le 5»/51 caliber gun américain, dont trois exemplaires furent livrés à la Grande-Bretagne pour servir d'artillerie côtière sous la dénomination 5»/51 BL Mark VI.

17. Cette pièce est habituellement connue sous le nom de Pom-pom gun.

18. Cette pièce est habituellement connue sous le nom de Pom-pom gun.

19. Ce canon était en service depuis 1904, mais transformé en arme anti-aérienne montée à l'arrière d'un camion pendant la guerre.

20. Nous n'avons pu identifier le nom russe de cette pièce, ni de traduction française certaine.

21. Le jeu World War I (2005) fait figurer un « obusier de $122 \mathrm{~mm}$ modèle 1900 » dans le matériel russe. Nous supposons qu'il s'agit du 122-mm gaubica obr. 1909 gg., mais comme son nom l'indique celui-ci ne date que de 1909. 


\section{TABLEAU 4}

Liste des véhicules terrestres représentés de façon identifiable dans les jeux du corpus.

\begin{tabular}{|c|c|c|c|c|c|c|c|c|c|c|c|}
\hline Pays & Type & Désignation (année) & 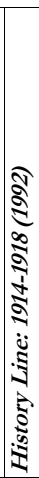 & 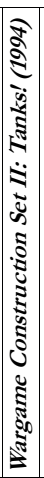 & 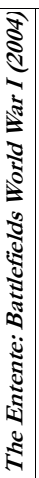 & 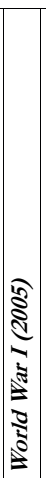 & 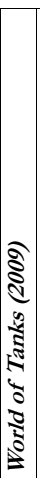 & 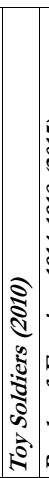 & 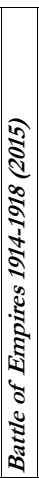 & 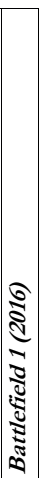 & 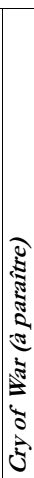 \\
\hline \multirow{9}{*}{ Allemagne } & \multirow{2}{*}{ Automitrailleuse } & Ehrhardt E-V/4 (1915) & $\mathrm{x}$ & & & & & & $\mathrm{x}$ & $\mathrm{x}$ & \\
\hline & & 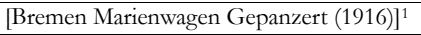 & & & & & & $\mathrm{x}$ & & & \\
\hline & Automobile & Mercedes 37/95 (1913) & & & & & & & & $\mathrm{x}$ & \\
\hline & \multirow{5}{*}{ Chars d'assaut } & Mark I (1916) ${ }^{2}$ & & & & & & $\mathrm{x}$ & & & \\
\hline & & Sturmpanzerwagen A7V (1917) & $\mathrm{x}$ & $\mathrm{x}$ & $\mathrm{x}$ & $\mathrm{x}$ & & $\mathrm{x}$ & $\mathrm{x}$ & $\mathrm{x}$ & $\mathrm{x}$ \\
\hline & & Mark IV $(1917)^{3}$ & & $\mathrm{x}$ & & & & & & & $\mathrm{x}$ \\
\hline & & [Leichter Kampfwagen (1918)] & & & & $\mathrm{x}$ & & & & & \\
\hline & & [Leichter Kampfwagen II (1918)] & & & $\mathrm{x}$ & & & & & & \\
\hline & Motocyclette & NSU 3 1/2 PS (1914) & & & & & & & & $\mathrm{x}$ & \\
\hline \multirow{3}{*}{ Autriche-Hongrie } & \multirow{2}{*}{ Automitrailleuse } & Austro-Daimler Panzerwagen (1904) & & & & $\mathrm{x}$ & & & & & \\
\hline & & Romfell Panzerwagen (1915) & & & & & & & $\mathrm{x}$ & $\mathrm{x}$ & \\
\hline & Automobile & Austro-Daimler Model 1909 (1909) & & & & & & & & $\mathrm{x}$ & \\
\hline Belgique & Automitrailleuse & Automitrailleuse Minerva (1914) & & & & $\mathrm{x}$ & & & & & \\
\hline \multirow{3}{*}{ États-Unis } & Automobile & Dodge Model 30 (1915) & & & & & & & & $\mathrm{x}$ & \\
\hline & Char d'assaut & Char Renault FT $(1918)^{4}$ & & $\mathrm{x}$ & & & & & & & \\
\hline & Motocyclette & Harley Davidson 18-J (1918) & & & & & & & & $\mathrm{x}$ & \\
\hline \multirow{5}{*}{ France } & Automitrailleuse & Charron modèle $1906(1906)^{5}$ & $\mathrm{x}$ & & & & & & $\mathrm{x}$ & & \\
\hline & \multirow{4}{*}{ Char d'assaut } & Char Schneider CA1 (1916) & & $\mathrm{x}$ & & $\mathrm{x}$ & & & $\mathrm{x}$ & & \\
\hline & & Saint-Chamond (1917) & $\mathrm{x}$ & $\mathrm{x}$ & & $\mathrm{x}$ & & & $\mathrm{x}$ & $\mathrm{x}$ & $\mathrm{x}$ \\
\hline & & 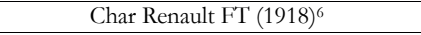 & $\mathrm{x}$ & $\mathrm{x}$ & $\mathrm{x}$ & & $\mathrm{x}$ & & $\mathrm{x}$ & $\mathrm{x}$ & $\mathrm{x}$ \\
\hline & & {$[\mathrm{FCM} 2 \mathrm{C}(1918)]^{7}$} & & & & & & & & $\mathrm{x}$ & \\
\hline \multirow{2}{*}{ Grande-Bretagne } & \multirow{2}{*}{ Automitrailleuse } & Rolls-Royce Armored Car (1914) & & & & & & & & $\mathrm{x}$ & \\
\hline & & Austin Armoured Car (1915) & & $\mathrm{x}$ & & $\mathrm{x}$ & & & & & \\
\hline
\end{tabular}

1. Nous plaçons ce véhicule dans les automitrailleuses en tant que prototype dérivé du Ehrhardt E-V/4, mais il s'agissait techniquement plutôt d'une chenillette.

2. Il s'agit de chars britanniques capturés et retournés contre leurs premiers propriétaires (désignés de manière générale par le terme de "Beutepanzer »).

3. Il s'agit de chars britanniques capturés et retournés contre leurs premiers propriétaires (désignés de manière générale par le terme de "Beutepanzer "). Le Mark IV existait en versions mâle et femelle, la première ayant un armement principal composé de deux canons et la seconde de six mitrailleuses. Le jeu Wargame Construction Set II: Tanks! (1994) fait la distinction entre les deux.

4. Il s'agit du char français Renault FT en service dans l'armée américaine. Cette dernière en produisit une copie, le M1917 light tank, qui n'arriva en Europe qu'après la fin des combats.

5. L'automitrailleuse Charron n'a été en service que jusqu'en 1911 et n’a donc pas vu la Première Guerre mondiale. Elle est toutefois incluse dans History Line: 1914-1918 (1992).

6. Ce char est généralement connu sous le nom de FT-17, bien que cette désignation soit anachronique. Il existait en deux versions, avec un canon ou une mitrailleuse. Les jeux Wargame Construction Set II: Tanks! (1994) et Battle of Empires 1914-1918 (2015) font la distinction entre les deux.

7. Ce char n'avait été que commandé en 1918 ; sa construction a débuté en 1919 avec une livraison en 1921. 


\begin{tabular}{|c|c|c|c|c|c|c|c|c|c|c|c|}
\hline & & Lanchester 4×2 Armoured Car (1915) & & & & $\mathrm{x}$ & $\mathrm{x}$ & & $\mathrm{x}$ & & \\
\hline & \multirow{7}{*}{ Char d'assaut } & [Little Willie (1915)] $^{8}$ & & & & $\mathrm{x}$ & & $\mathrm{x}$ & & & \\
\hline & & Mark I (1916) & $\mathrm{x}$ & & $\mathrm{x}$ & $\mathrm{x}$ & $\mathrm{x}$ & $\mathrm{x}$ & $\mathrm{x}$ & & \\
\hline & & Mark IV $(1917)^{9}$ & $\mathrm{x}$ & $\mathrm{x}$ & & & & & $\mathrm{x}$ & & $\mathrm{x}$ \\
\hline & & [Mark B (1917)] & & & & & & & $\mathrm{x}$ & & \\
\hline & & Mark V (1918) & & $\mathrm{x}$ & & $\mathrm{x}$ & & & & $\mathrm{x}$ & \\
\hline & & Mark A Whippet (1917) & & $\mathrm{x}$ & $\mathrm{x}$ & $\mathrm{x}$ & & $\mathrm{x}$ & $\mathrm{x}$ & & \\
\hline & & Mark VIII (1918) & & & & & & & $\mathrm{x}$ & & \\
\hline Italie & Automitrailleuse & FIAT-Terni « Tripoli » (1918) & & & & & & & & $\mathrm{x}$ & \\
\hline \multirow{9}{*}{ Russie } & \multirow{5}{*}{ Automitrailleuse } & Austin Armoured Car (1915) ${ }^{10}$ & & & & $\mathrm{x}$ & & & $\mathrm{x}$ & & \\
\hline & & Austin-Putilov (1915) $)^{11}$ & & & & $\mathrm{x}$ & & & & & \\
\hline & & Lanchester 4×2 Armoured Car (1915) ${ }^{12}$ & & & & & & & $\mathrm{x}$ & & \\
\hline & & Mgebrov-Renault (1915) & & & & & & & $\mathrm{x}$ & & \\
\hline & & Mgebrov-White (1915) & & & & $\mathrm{x}$ & & & & & \\
\hline & Char d'assaut & 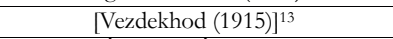 & & & & $\mathrm{x}$ & & & & & \\
\hline & Véhicule blindé14 & Gárford-Putilov (1915) & & & & $\mathrm{x}$ & & & $\mathrm{x}$ & $\mathrm{x}$ & \\
\hline & Véhicule de transport & Russo-Balt T 40/65 (1913) ${ }^{15}$ & & & & $\mathrm{x}$ & & & & & \\
\hline & Train blindé & Zaamurets (1916) & & & & & & & & $\mathrm{x}$ & \\
\hline
\end{tabular}

8. Le Little Willie était un prototype construit à un seul exemplaire et n'ayant jamais servi opérationnellement. 9. Le Mark IV existait en versions mâle et femelle, la première ayant un armement principal composé de deux canons et la seconde de six mitrailleuses. Le jeu Wargame Construction Set II: Tanks! (1994) fait la distinction entre les deux.

10. Véhicule fourni à la Russie par l'armée britannique.

11. Il s'agit d'une version légèrement remaniée et produite en Russie de la Austin Armoured Car britannique.

12. Véhicule de fourniture britannique.

13. Il s'agit d'un projet de char qui ne dépassa pas le stade de prototype.

14. Le Gárford-Putílov se situe à mi-chemin entre l'automitrailleuse et le char : développé comme un camion blindé sur roues, il n'en est pas moins équipé d'un canon en plus de deux à trois mitrailleuses.

15. Simple camion à l'origine, celui-ci pouvait se voir doté d'un armement sur la plate-forme arrière. 


\section{TABLEAU 5}

Liste des appareils volants représentés de façon identifiable dans les jeux du corpus.

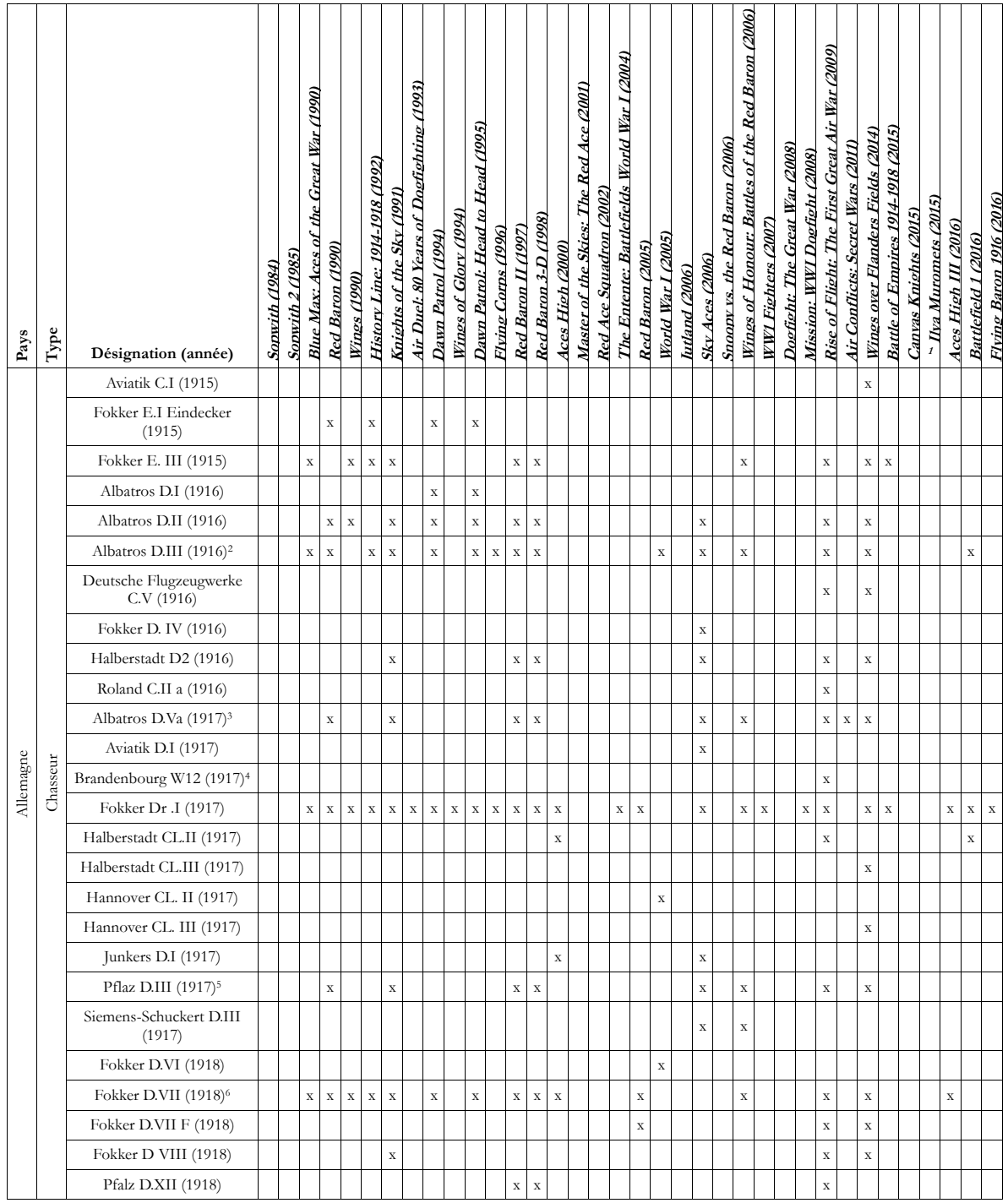

1. Les informations sont quasiment introuvables concernant ce jeu, nous ne mentionnons donc que la présence évidente du Ilya Muromets.

2. Le jeu Wings over Flanders Fields (2014) propose trois versions de cet appareil.

3. Le jeu Wings over Flanders Fields (2014) propose quatre versions de cet appareil.

4. Il s'agit d'un hydravion.

5. Le jeu Rise of Flight: The First Great Air War (2009) propose deux versions de cet appareil.

6. Le jeu Wings over Flanders Fields (2014) propose trois versions de cet appareil. 


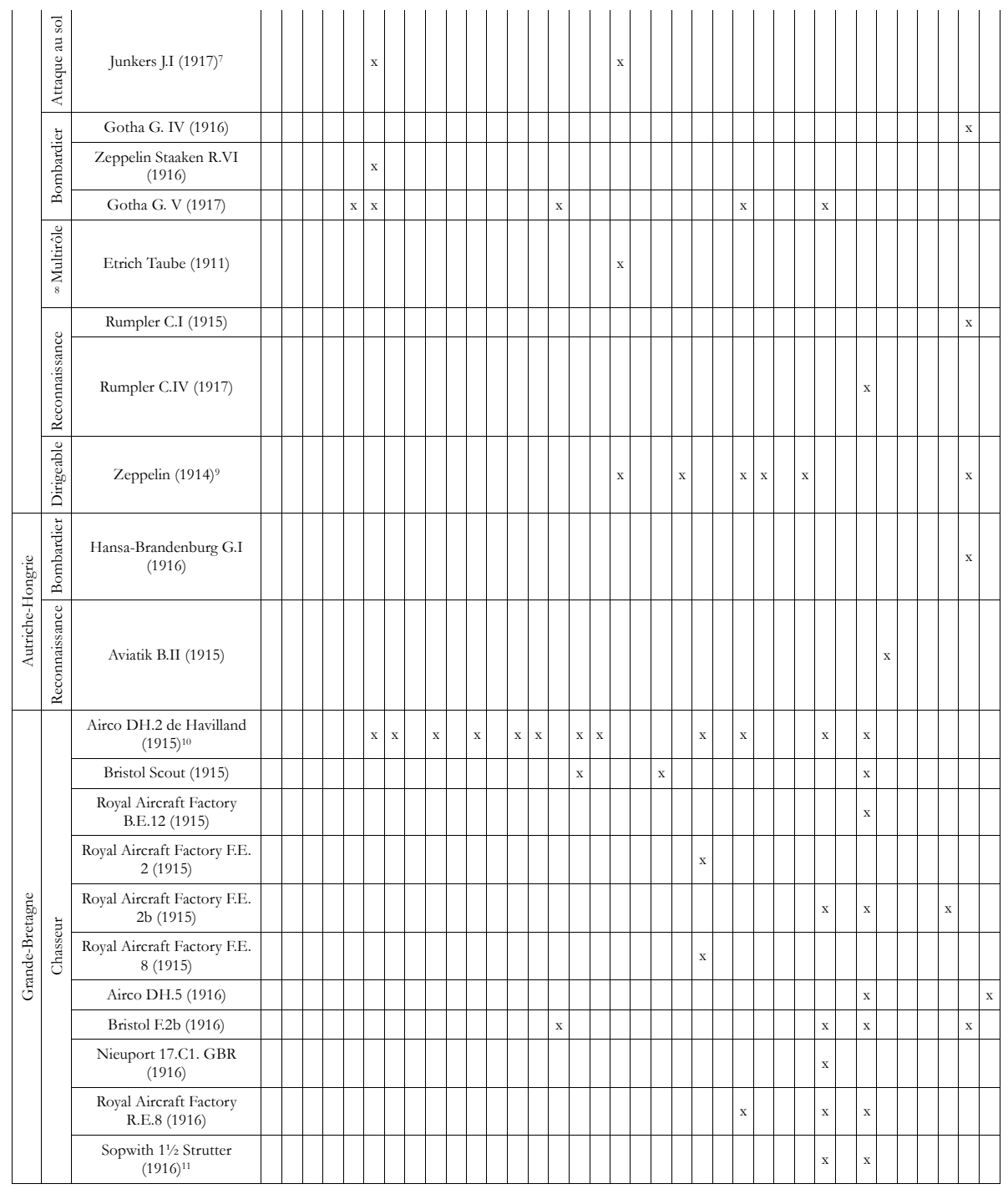

7. Cet appareil correspondait au Junkers J 4 dans la nomenclature interne de l'entreprise Junkers, et est désigné sous ce nom dans History Line: 1914-1918 (1992).

8. Dépourvu d'armement, le Etrich Taube fut avant tout utilisé pour la reconnaissance. Toutefois, en l'absence d'appareils spécifiques, il fut également employé pour la chasse et le bombardement dans les premiers mois du conflit.

9. Le terme Zeppelin désigne une entreprise ayant produit des avions et des dirigeables. Son emploi pour désigner de manière générique les dirigeables allemands est donc très approximatif, chacun d'entre eux étant différent à la manière des navires. Quelques jeux, notamment Jutland (2006), distinguent les modèles particuliers.

10. Le jeu Wings over Flanders Fields (2014) propose trois versions de cet appareil.

11. Le jeu Wings over Flanders Fields (2014) propose deux versions de cet appareil. 


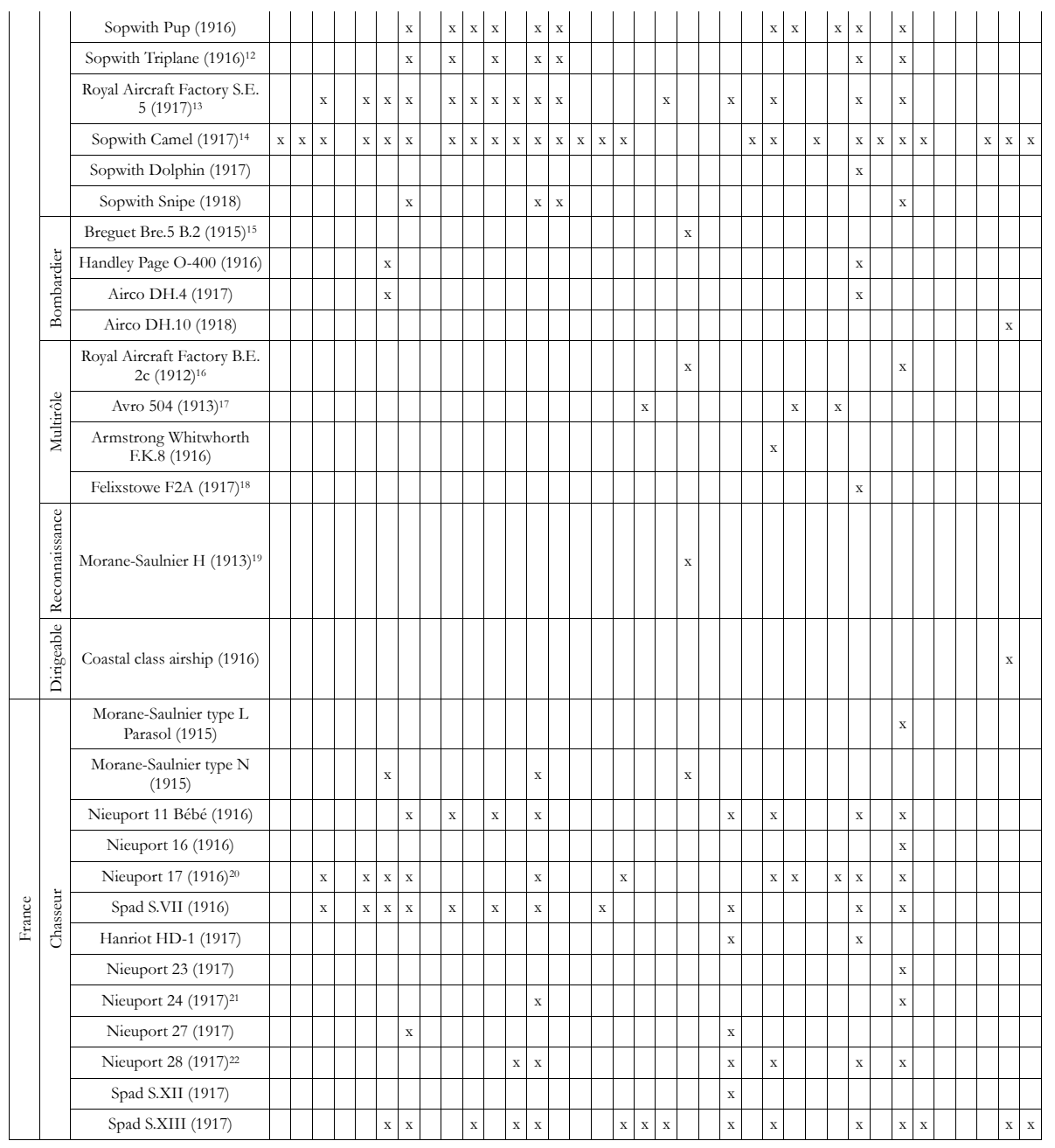

12. Le jeu Wings over Flanders Fields (2014) propose deux versions de cet appareil.

13. Le jeu Wings over Flanders Fields (2014) propose trois versions de cet appareil.

14. Le jeu Wings over Flanders Fields (2014) propose deux versions de cet appareil.

15. Il s'agit d'un appareil français fourni à l'armée britannique.

16. Le jeu Wings over Flanders Fields (2014) propose deux versions de cet appareil.

17. Trop ancien pour être employé comme chasseur sur le front de la Grande Guerre, le Avro 504 n'en a pas

moins été produit à presque 9000 exemplaires, avec plus d'une vingtaine de versions employées pas près

d'une quarantaine de pays, dans virtuellement tous les rôles imaginables à l'époque.

18. Il s'agit d'un hydravion.

19. Il s'agit d'un appareil français produit sous licence en Grande-Bretagne.

20. Le jeu Wings over Flanders Fields (2014) propose trois versions de cet appareil.

21. Le jeu Wings over Flanders Fields (2014) propose quatre versions de cet appareil.

22. Le jeu Rise of Flight: The First Great Air War (2009) propose deux versions de cet appareil. 


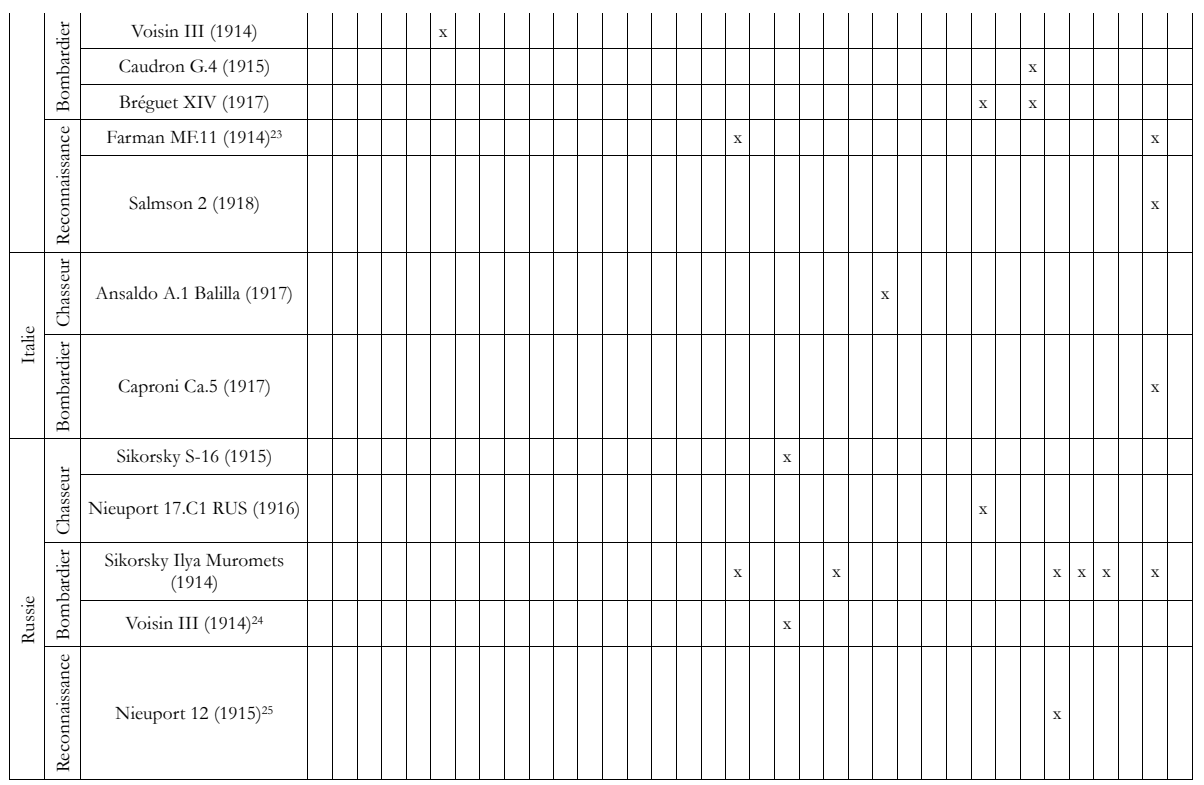

23. Le jeu World War I (2005) comporte pour l'armée russe un avion de bombardement et reconnaissance "MF22 " que nous ne sommes pas parvenu à identifier. Le graphisme le fait cependant ressembler à un Farman français de la fin de la guerre, possiblement un F.40 de 1917, sans que nous puissions établir la raison de sa présence comme unité russe.

24. Appareil français fourni à l'armée russe.

25. Appareil français fourni à l'armée russe. 


\section{TABLEAU 6}

Liste des navires représentés de façon identifiable dans les jeux du corpus. Les noms donnés désignent plus généralement des classes que des bâtiments individuels.

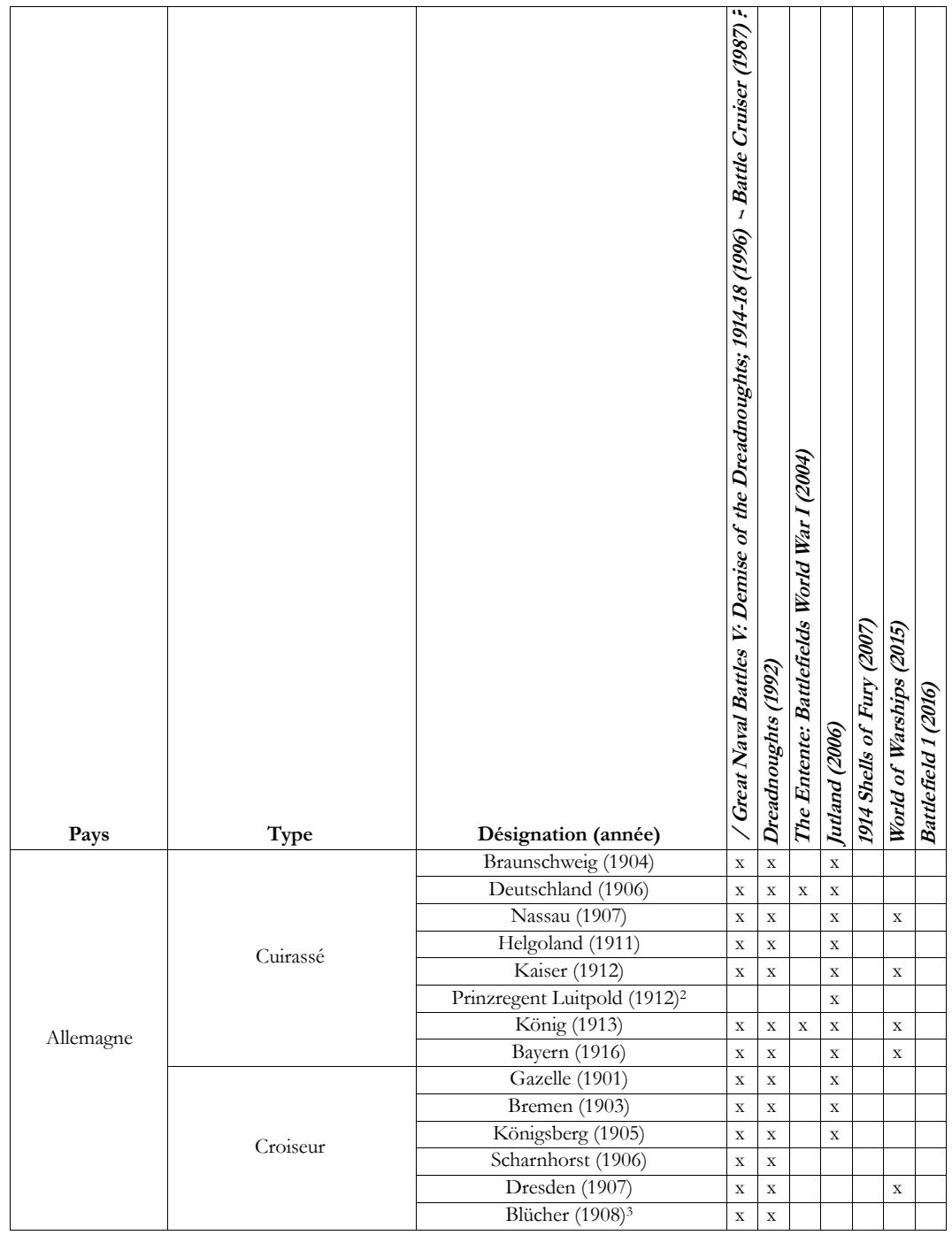

1. Nous n'avons pu nous procurer la liste des unités disponibles dans Battle Cruiser (1987). Toutefois, leur nombre nous est connu, et est quasiment identique à celui de Great Naval Battles V: Demise of the Dreadnoughts; 1914-18 (1996). Sachant que les deux jeux ont été publiés par le même éditeur bien qu'à une décennie d'intervalle, exactement sur le même sujet, et que cette liste représente la quasi-totalité des bâtiments en service à l'époque dans les marines allemande et britannique (au moins pour les cuirassés et croiseurs), il est probable que les deux jeux aient proposé sensiblement les mêmes unités.

2. Cuirassé de la classe Kaiser, légèrement différent de ses sister-ships.

3. Le Blücher était un croiseur cuirassé. Il est classé parmi les cuirassés dans Great Naval Battles V: Demise of the Dreadnoughts; 1914-18 (1996).

4. Le Von der Tann était un croiseur de bataille. Il est classé parmi les cuirassés dans Great Naval Battles V: Demise of the Dreadnoughts; 1914-18 (1996). 


\begin{tabular}{|c|c|c|c|c|c|}
\hline & Kolberg (1908) & $x$ & $\mathrm{x}$ & & $\mathrm{x}$ \\
\hline & Von der Tann $(1909)^{4}$ & $\mathrm{x}$ & $\mathrm{x}$ & $\mathrm{x}$ & \\
\hline & Moltke $(1910)^{5}$ & $\mathrm{x}$ & $\mathrm{x}$ & $\mathrm{x}$ & \\
\hline & Magdeburg (1911) & $\mathrm{x}$ & $\mathrm{x}$ & $\mathrm{x}$ & \\
\hline & Karlsruhe (1912) & $x$ & $\mathrm{x}$ & $\mathrm{x}$ & $x$ \\
\hline & Seydlitz $(1912)^{6}$ & $\mathrm{x}$ & $\mathrm{x}$ & $\mathrm{x}$ & \\
\hline & Derfflinger $(1913)^{7}$ & $\mathrm{x}$ & $\mathrm{x}$ & $\mathrm{x}$ & \\
\hline & Graudenz (1913) & & $\mathrm{x}$ & $\mathrm{x}$ & \\
\hline & Lützow (1913) & & $\mathrm{x}$ & & \\
\hline & Pillau (1914) & $\mathrm{x}$ & $\mathrm{x}$ & $\mathrm{x}$ & \\
\hline & Brummer (1915) & $\mathrm{x}$ & $\mathrm{x}$ & $\mathrm{x}$ & \\
\hline & Hindenburg $(1915)^{8}$ & $\mathrm{x}$ & $\mathrm{x}$ & & \\
\hline & Wiesbaden (1915) & $\mathrm{x}$ & $\mathrm{x}$ & $\mathrm{x}$ & \\
\hline & Königsberg (1915) & $\mathrm{x}$ & $\mathrm{x}$ & $\mathrm{x}$ & \\
\hline & Cöln (1916) & $x$ & $\mathrm{x}$ & & \\
\hline & Mackensen $(1917)^{9}$ & $\mathrm{x}$ & $\mathrm{x}$ & 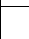 & \\
\hline & Möwe (1879) & & & $\mathrm{x}$ & \\
\hline Croicen reviliaite10 & Wolf (1913) & & & $\mathrm{x}$ & \\
\hline Crosseur auxiliaire ${ }^{10}$ & Wolf II (1913) & & & $\mathrm{x}$ & \\
\hline & Greif (1914) & & & $\mathrm{x}$ & \\
\hline Croiceur mouilleur de mines & Pelikan (1891) & & & $\mathrm{x}$ & \\
\hline Croiseur mouilleur de mines & Nautilus (1907) & & & $\mathrm{x}$ & \\
\hline & G132 (1906) & $\mathrm{x}$ & $\mathrm{x}$ & $\mathrm{x}$ & \\
\hline & G138 (1906) & & $\mathrm{x}$ & & \\
\hline & V150 (1907) & $\mathrm{x}$ & $\mathrm{x}$ & $\mathrm{x}$ & \\
\hline & G169 (1908) & $\mathrm{x}$ & & $\mathrm{x}$ & \\
\hline & S138 (1908) & & & $\mathrm{x}$ & \\
\hline & V156 (1908) & & & $\mathrm{x}$ & \\
\hline & V161 (1908) & & & $\mathrm{x}$ & \\
\hline Dectsoner11 & V162 (1908) & & $\mathrm{x}$ & $\mathrm{x}$ & \\
\hline Destroyer ${ }^{11}$ & S165 (1909) & & $\mathrm{x}$ & & \\
\hline & V180 (1909) & $x$ & & $\mathrm{x}$ & \\
\hline & G192 (1910) & & & $\mathrm{x}$ & \\
\hline & S13 (1911) & & & $\mathrm{x}$ & \\
\hline & V1 (1911) & $x$ & $\mathrm{x}$ & $\mathrm{x}$ & \\
\hline & G37 (1913) & & & $\mathrm{x}$ & \\
\hline & V25 (1913) & $x$ & $x$ & $\mathrm{x}$ & $x$ \\
\hline & B97 (1914) & & $\mathrm{x}$ & $\mathrm{x}$ & \\
\hline
\end{tabular}

5. Les navires de classe Moltke étaient des croiseurs de bataille. Ils sont classés parmi les cuirassés dans Great Naval Battles V: Demise of the Dreadnoughts; 1914-18 (1996).

6. Le Seydlitz était un croiseur de bataille. Il est classé parmi les cuirassés dans Great Naval Battles V: Demise of the Dreadnoughts; 1914-18 (1996).

7. Les navires de classe Derfflinger étaient des croiseurs de bataille. Ils sont classés parmi les cuirassés dans Great Naval Battles V: Demise of the Dreadnoughts; 1914-18 (1996).

8. Le Hindenburg était l'un des croiseurs de bataille de la classe Derfflinger et est en conséquence classé parmi les cuirassés dans Great Naval Battles V: Demise of the Dreadnoughts; 1914-18 (1996) (cf. note précédente).

9. Les navires de classe Mackensen étaient des croiseurs de bataille. Ils sont classés parmi les cuirassés dans Great Naval Battles V: Demise of the Dreadnoughts; 1914-18 (1996).

10. Les « croiseurs auxiliaires " (désignés sous le terme de " croiseurs armés " pendant la Grande Guerre) correspondent à des navires marchands armés pour la guerre de course, soit des actions de corsairerie visant à déstabiliser le commerce ennemi.

11. Ces bâtiments allemands étaient pour la plupart désignés comme " navires torpilleurs de haute mer ", soit un rôle quasiment équivalent à celui de destroyers. 


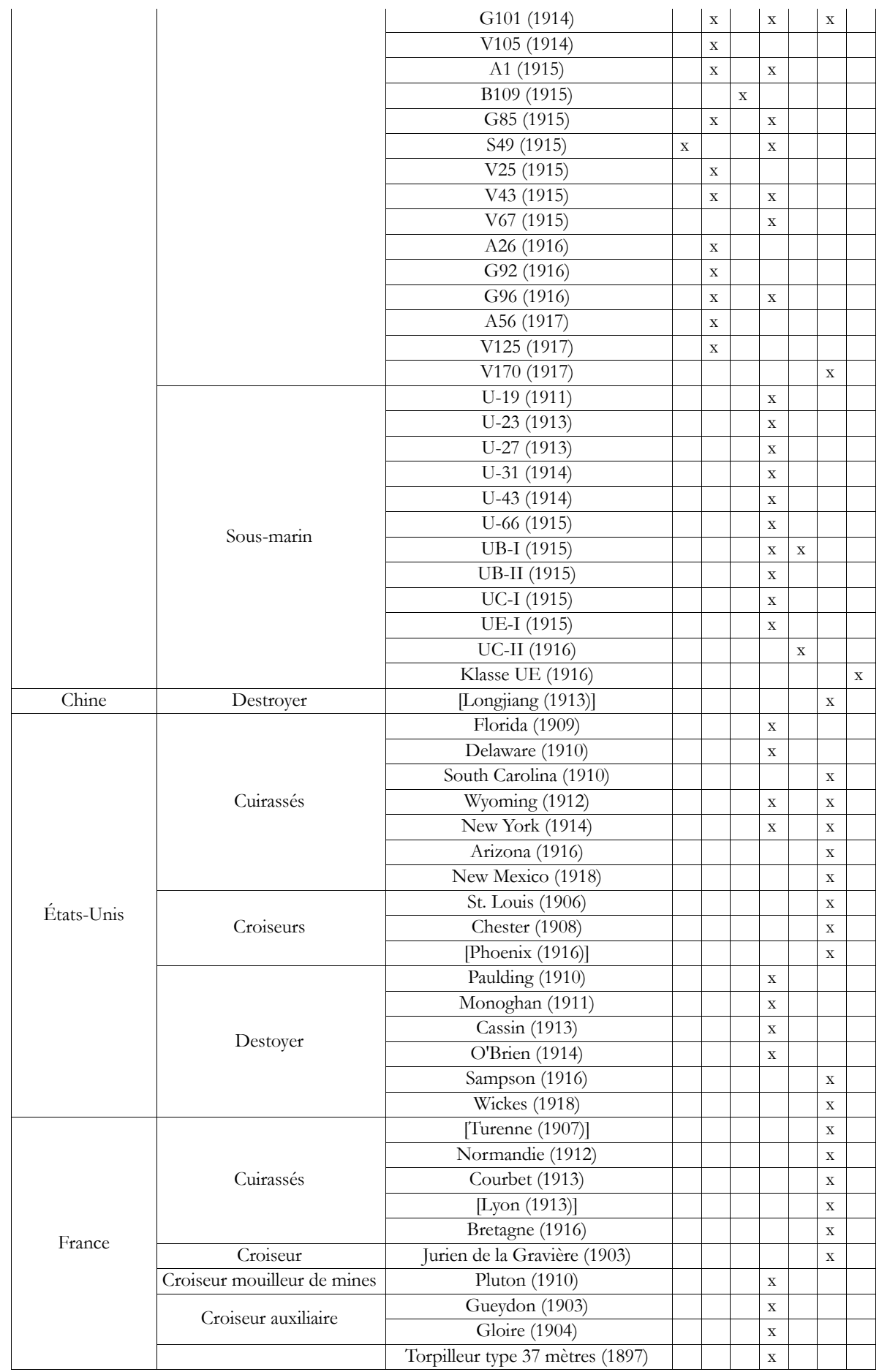




\begin{tabular}{|c|c|c|c|c|c|c|c|}
\hline & & Cyclone (1898) & & & $\mathrm{x}$ & & \\
\hline & & Dunois (1898) & & & $\mathrm{x}$ & & \\
\hline & & Durandal (1899) & & & $\mathrm{x}$ & & \\
\hline & & Pertuisane $(1900)^{13}$ & & & $\mathrm{x}$ & & \\
\hline & & Mistral (1901) & & & $\mathrm{x}$ & & \\
\hline & Dectrourer 12 & Arquebuse (1902) & & & $\mathrm{x}$ & & \\
\hline & Destroyer & Claymore (1905) & & & $\mathrm{x}$ & & \\
\hline & & Torpilleur type 38 mètres (1905) & & & $\mathrm{x}$ & & \\
\hline & & Branlebas (1907) & & & $\mathrm{x}$ & & \\
\hline & & Bouclier (1910) & & & $\mathrm{x}$ & & \\
\hline & & Bisson (1911) & & & $\mathrm{x}$ & & \\
\hline & & Enseigne Roux (1913) & & & $\mathrm{x}$ & & \\
\hline & & Sirène (1901) & & & $\mathrm{x}$ & & \\
\hline & Sous-marin & Aigrette (1904) & & & $\mathrm{x}$ & & \\
\hline & sous-marin & Pluviôse (1907) & & & $\mathrm{x}$ & & \\
\hline & & Amiral Bourgeois (1912) & & & $\mathrm{x}$ & & \\
\hline & & Majestic (1896) & $\mathrm{x}$ & & & & \\
\hline & & Canopus (1898) & $\mathrm{x}$ & $\mathrm{x}$ & & & \\
\hline & & Formidable (1898) & $\mathrm{x}$ & & & & \\
\hline & & London (1899) & $\mathrm{x}$ & & & & \\
\hline & & Duncan (1901) & $\mathrm{x}$ & & - & & \\
\hline & & King Edward VII (1903) & $\mathrm{x}$ & & $\mathrm{x}$ & & \\
\hline & & Lord Nelson (1906) & $\mathrm{x}$ & 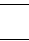 & & & \\
\hline & & Dreadnought (1906) & $\mathrm{x}$ & $\mathrm{x}$ & $\mathrm{x}$ & & $\mathrm{x}$ \\
\hline & & Bellerophon (1907) & $\mathrm{x}$ & $\mathrm{x}$ & $\mathrm{x}$ & $\mathrm{x}$ & \\
\hline & & St. Vincent (1908) & $\mathrm{x}$ & $\mathrm{x}$ & $\mathrm{x}$ & & \\
\hline & & Neptune (1909) & $\mathrm{x}$ & $\mathrm{x}$ & $\mathrm{x}$ & & \\
\hline & Cuiracsé & Colossus (1911) & $\mathrm{x}$ & $\mathrm{x}$ & $\mathrm{x}$ & & \\
\hline & Cuirasse & Orion (1911) & $\mathrm{x}$ & $\mathrm{x}$ & $\mathrm{x}$ & $\mathrm{x}$ & \\
\hline & & King George V (1911) & $\mathrm{x}$ & $\mathrm{x}$ & $\mathrm{x}$ & & \\
\hline Grande-Bretagne & & Iron Duke (1912) & $\mathrm{x}$ & $\mathrm{x}$ & $\mathrm{x}$ & $\mathrm{x}$ & \\
\hline & & Queen Elizabeth (1913) & $\mathrm{x}$ & $\mathrm{x}$ & $\mathrm{x}$ & $\mathrm{x}$ & \\
\hline & & Erin (1913) & $\mathrm{x}$ & $\mathrm{x}$ & $\mathrm{x}$ & & \\
\hline & & Agincourt (1913) & $\mathrm{x}$ & $\mathrm{x}$ & $\mathrm{x}$ & & \\
\hline & & Canada (1913) & $\mathrm{x}$ & $\mathrm{x}$ & $\mathrm{x}$ & & \\
\hline & & Revenge (1914) & $\mathrm{x}$ & $\mathrm{x}$ & $\mathrm{x}$ & & \\
\hline & & Lord Clive (1915) ${ }^{14}$ & & & $\mathrm{x}$ & & \\
\hline & & M-15 class $(1915)^{15}$ & & & $\mathrm{x}$ & & \\
\hline & & Warspite (1915) & & & & $\mathrm{x}$ & \\
\hline & & Erebus $(1916)^{16}$ & & & $\mathrm{x}$ & & \\
\hline & & Cressy (1899) & $\mathrm{x}$ & & & & \\
\hline & & Drake (1901) & $\mathrm{x}$ & $\mathrm{x}$ & & & \\
\hline & Croiseur & Monmouth (1901) & $\mathrm{x}$ & $\mathrm{x}$ & $\mathrm{x}$ & & \\
\hline & & Devonshire (1903) & $\mathrm{x}$ & $\mathrm{x}$ & $\mathrm{x}$ & & \\
\hline & & Duke of Edinburgh (1904) & $\mathrm{x}$ & $\mathrm{x}$ & $\mathrm{x}$ & & \\
\hline
\end{tabular}

12. Ces bâtiments français étaient désignés selon le cas comme " torpilleurs " ou " contre-torpilleurs ", soit des rôles quasiment équivalent à celui de destroyers.

13. Cette classe est plus connue sous le nom de Rochefortais.

14. Les bâtiments de classe Lord Clive n'étaient pas à proprement parler des cuirassés, mais des monitors employés comme artillerie côtière flottante.

15. Les bâtiments de classe M-15 n'étaient pas à proprement parler des cuirassés, mais des monitors employés comme artillerie côtière flottante.

16. Les bâtiments de classe Erebus n'étaient pas à proprement parler des cuirassés, mais des monitors employés comme artillerie côtière flottante. 


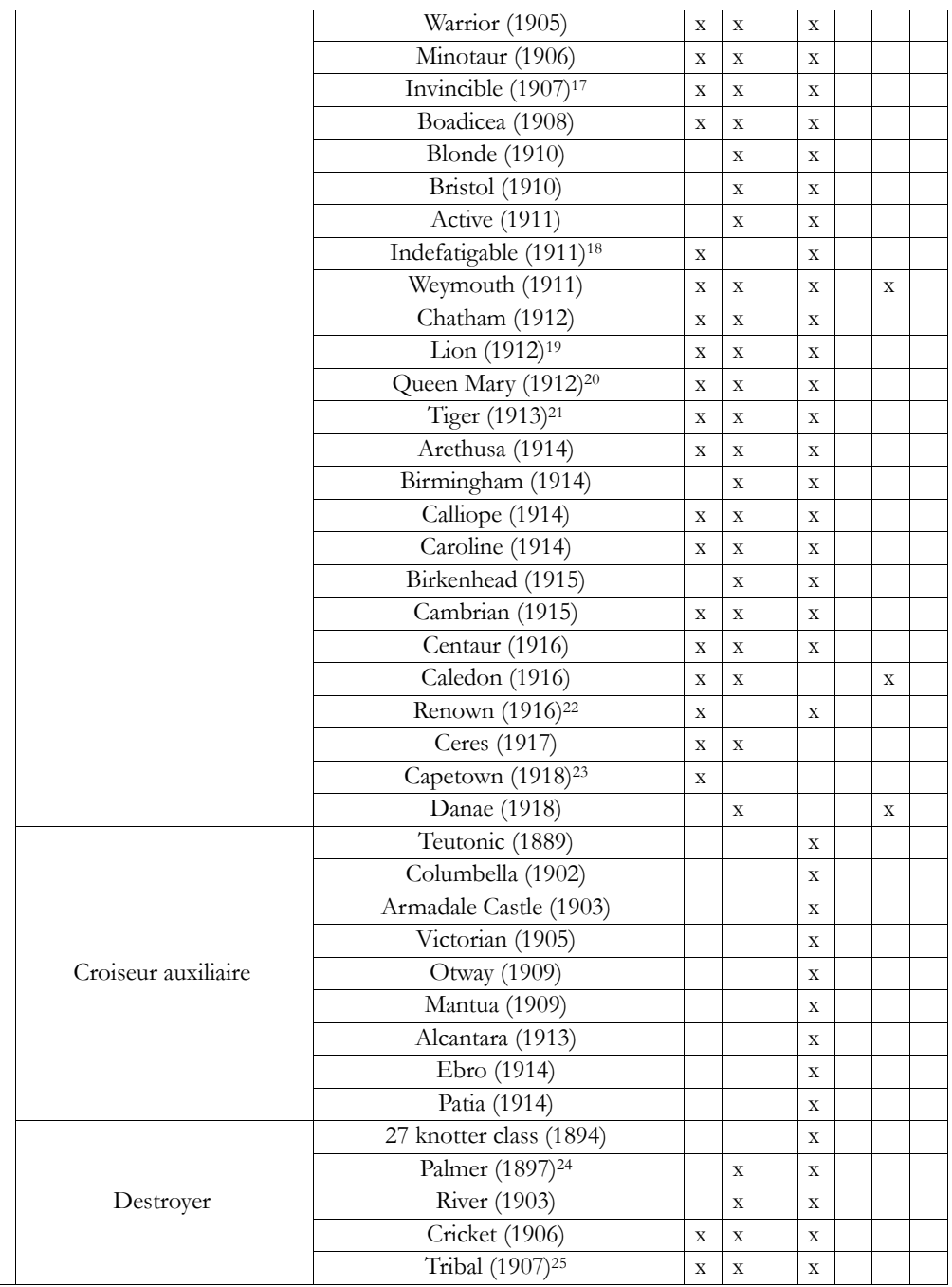

17. Les navires de classe Invincible étaient des croiseurs de bataille. Ils sont classés parmi les cuirassés dans Great Naval Battles V: Demise of the Dreadnoughts; 1914-18 (1996).

18. Les navires de classe Indefatigable étaient des croiseurs de bataille. Ils sont classés parmi les cuirassés dans Great Naval Battles V: Demise of the Dreadnoughts; 1914-18 (1996).

19. Les navires de classe Lion étaient des croiseurs de bataille. Ils sont classés parmi les cuirassés dans Great Naval Battles V: Demise of the Dreadnoughts; 1914-18 (1996).

20. Les navires de classe Queen Mary étaient des croiseurs de bataille. Ils sont classés parmi les cuirassés dans Great Naval Battles V: Demise of the Dreadnoughts; 1914-18 (1996).

21. Les navires de classe Tiger étaient des croiseurs de bataille. Ils sont classés parmi les cuirassés dans Great Naval Battles V: Demise of the Dreadnoughts; 1914-18 (1996).

22. Les navires de classe Renown étaient des croiseurs de bataille. Ils sont classés parmi les cuirassés dans Great Naval Battles V: Demise of the Dreadnoughts; 1914-18 (1996).

23. Le croiseur léger Capetown a été lancé en 1919. Il appartenait toutefois au groupe Carlisle de la C-class, dont la tête de série fut lancée en 1918.

24. Le jeu Jutland (2006) propose plus généralement la 30 knotter class, dont le Palmer est un représentant.

25. Le jeu Jutland (2006) distingue les classes Tribal A et B, la seconde étant une amélioration de la première. 


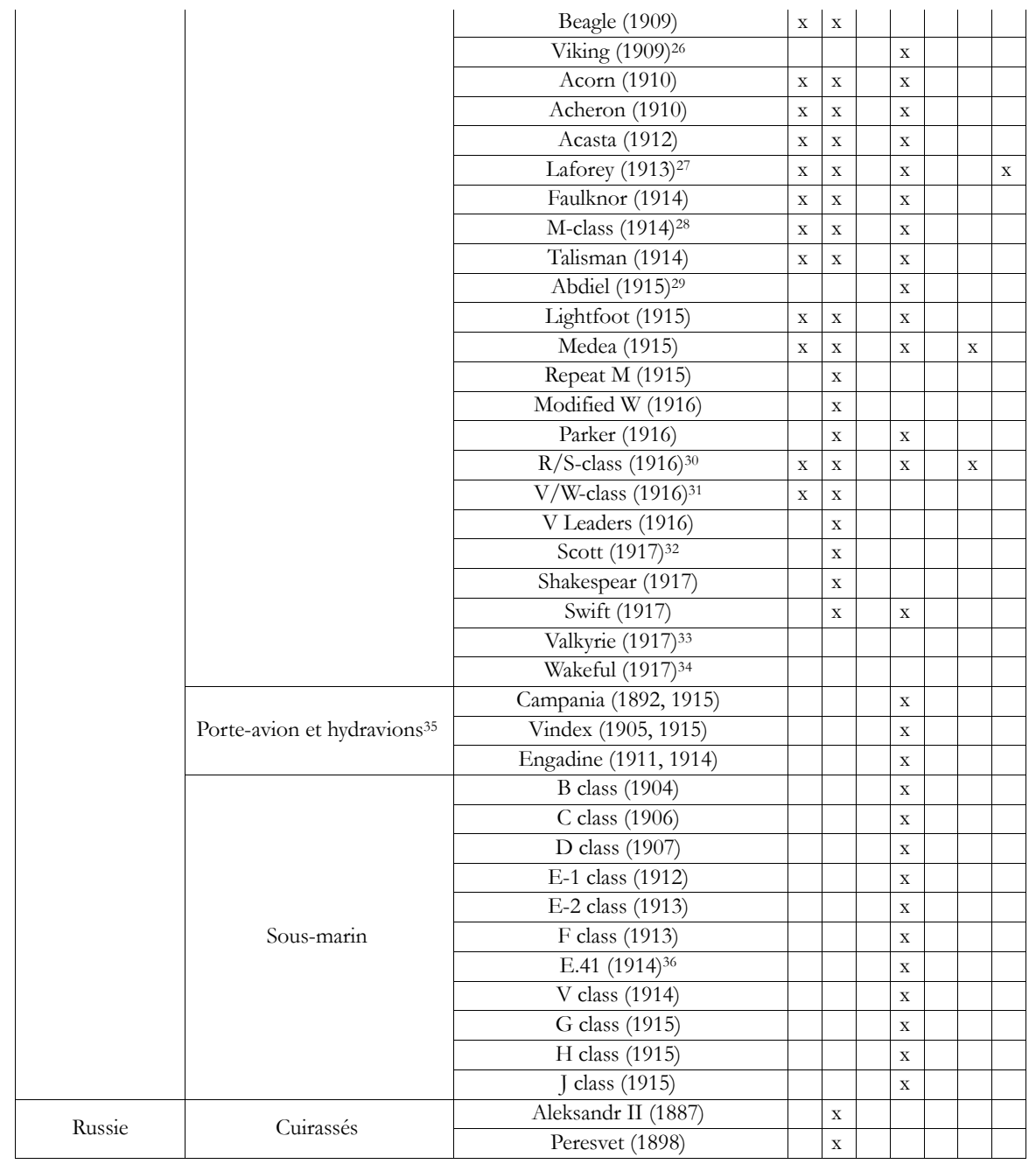

26. Destroyer de classe Tribal $\mathrm{B}$, amélioré par rapport à ses sister-ship.

27. Le jeu Dreadnought (1992) traite séparément la L-class en général et le Laforey, qui était sa tête de série. Le jeu Jutland (2006) distingue les destroyer Laforey à deux et trois cheminées.

28. Le jeu Jutland (2006) distingue les destroyer de M-class à deux et trois cheminées.

29. Destroyer de classe Lightfoot modifié pour le mouillage de mines.

30. Les classes $R$ et $S$ de destroyers britanniques sont différentes, toutes deux lancées en 1916. Elles sont disjointes dans Dreadnoughts (1992) mais regroupées dans Great Naval Battles V: Demise of the Dreadnoughts; 1914-18 (1996). Le jeu World of Warships (2015) propose un destroyer de classe R intégré dans la marine thailandaise sous le nom de Phra Ruang.

31. Les classes V et W de destroyers britanniques sont différentes, toutes deux lancées en 1916. Elles sont disjointes dans Dreadnoughts (1992) mais regroupées dans Great Naval Battles V: Demise of the Dreadnoughts; 1914-18 (1996).

32. Le Scott faisait partie de la classe V Leaders. Toutefois, le jeu Dreadnoughts (1992) traite les deux séparément. 33. Le Valkyrie était un bâtiment de classe V Leaders.

34. Le Wakeful était un bâtiment de classe W.

35. Ces bâtiments ont tous été transformés en porte-avions à partir de bâtiments civils. Les modifications étant importantes, nous indiquons leur date de lancement et leur date de conversion.

36. Le E.41 était un sous-marin de classe E-2 modifié pour pouvoir mouiller des mines. 


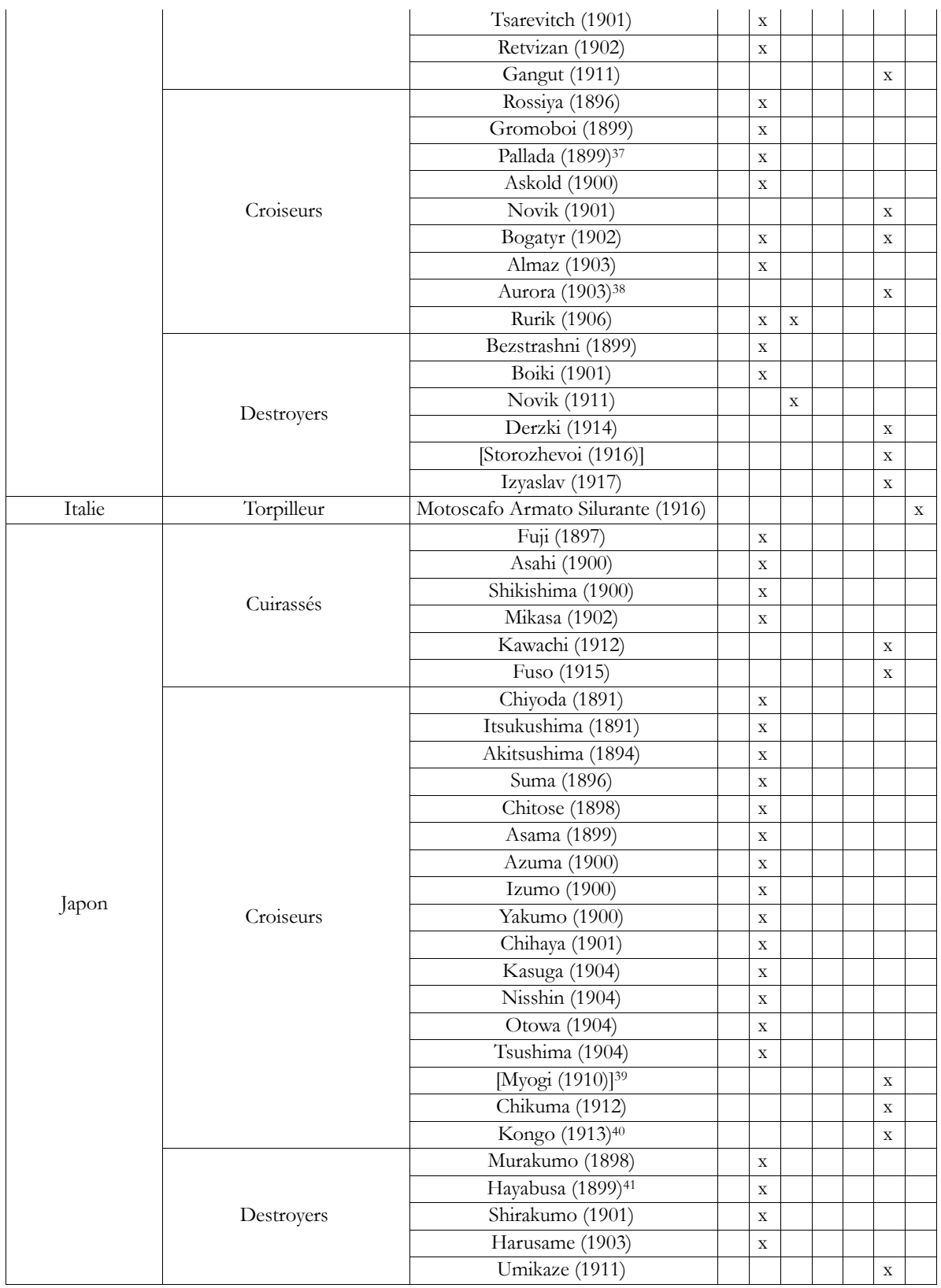

37. Le Pallada fut coulé à Port-Arthur en 1904. Il fut ensuite renfloué par le Japon et, au moment de la Première

Guerre mondiale, servait dans la marine japonaise sous le nom de Tsugaru.

38. L'Aurora était un croiseur protégé de classe Pallada.

39. Le Myogi était un projet de croiseur de bataille. Le jeu World of Warships (2015) le classe dans les cuirassés. 40. Le Kongo était un croiseur de bataille. Le jeu World of Warships (2015) le classe dans les cuirassés.

41. Le Hayabusa était un torpilleur, mais le jeu Dreadnoughts (1992) le classe dans les destroyers. 


\begin{tabular}{|l|l|l|l|l|l|l|l|l|} 
& & Isokaze (1917) & & & $\mathrm{x}$ & \\
\cline { 2 - 8 } & \multirow{3}{*}{ Canonnières } & Maya (1886) & & $\mathrm{x}$ & & & & \\
\cline { 2 - 9 } & Tatsuta (1894) & $\mathrm{x}$ & & & & \\
\hline & Uji (1903) & $\mathrm{x}$ & & & & \\
\hline
\end{tabular}

42. Le Tatsuta était initialement un croiseur, mais a été reclassé en canonnière en 1912. 